\title{
TRAIT-BASED INDIVIDUAL DIFFERENCES ON DISCOMFORT GLARE RATING RESPONSES AND RELATED VISUAL CONTRAST SENSITIVITY
}

BY

\author{
HARUETAI MEKAROONREUNG
}

\author{
A THESIS \\ IN \\ INDUSTRIAL AND SYSTEMS ENGINEERING \\ Submitted to the Graduate Faculty \\ of Virginia Polytechnic Institute and State University in \\ Partial Fulfillment of the Requirements for the Degree of
}

\author{
MASTER OF SCIENCE IN \\ INDUSTRIAL AND SYSTEMS ENGINEERING
}

Dr. Thurmon E. Lockhart, Chair

Dr. Tonya L. Smith-Jackson

Dr. Suzanne E. Lee

July 10, 2003

Blacksburg, Virginia

Keywords: Trait, Extraversion, Neuroticism, Glare Discomfort, Subjective Rating, Contrast Sensitivity 


\title{
Trait-Based Individual Differences on Discomfort Glare Rating Responses and Related Visual Contrast Sensitivity
}

\author{
Haruetai Mekaroonreung
}

\section{(ABSTRACT)}

This research was designed to investigate the relationship between Trait-based Individual differences (neuroticism and extraversion) and glare subjective responses as well as the actual contrast sensitivity when exposed to the same manipulated glare condition. In addition, the relationship between the glare subjective responses and actual contrast sensitivity was investigated. To examine the trait-based individual differences, the International Personality Item Pool (IPIP) was used while the subjective glare experience was examined utilizing modified glare discomfort rating scale. The visual performance was measured through the contrast sensitivity level using adjustable contrast level of the Landolt's C target. This investigation compared 36 individuals (9 high neuroticism scorers, 9 low neuroticism scorers, 9 high extraversion scorers, and 9 low extraversion scorers) on subjective discomfort glare rating responses and visual contrast sensitivity. The study is directed toward improving our understanding of influencing factors on the experience of discomfort glare, which may eventually be applied to the design of glare measurement methods, and toward training and selection of drivers and workers who may work under conditions of glare. Results indicated significant effect of extraversion trait on rating response while insignificant effect on visual related performance was found. The relationships between rating response and visual performance were also found to be quite low in this study. In conclusion, the expected model was supported but only on the extraversion trait. 


\section{ACKNOWLEDGEMENTS}

Many people have made it possible for this research to be successful. Most of all, I would especially like to thank my advisor, Dr. Thurmon Lockhart, for his valuable supports and encouragement in countless ways. His devoted time and patience are most appreciated. I would also like to thank my committee, Dr. Tonya Smith-Jackson, and Dr. Suzanne Lee, for their indispensable feedback, materials, support, and encouragement throughout the year. Without all of you, this work would not have been possible.

Additional thanks are devoted to Lovedia Cole, Theresa Coalson, and everyone else who assisted me through this academic process. My memorable friends in the Thai Student Association as well as the Human Factors Engineering program are the other grateful pieces of my years in the U.S. My work will not be accomplished without all participants who participated in this research. Their devoted time and effort for the study are recognized.

Further appreciation is given to the Fulbright and the P.E.O. foundation for sponsoring my academic study in the U.S.

Last but not least, special thanks and love to my family, beloved roommate, Lisa Patvivasiri, and especially to my fiancé, Kasem Lohasiriwat, for being there with their loves, everlasting devotions, encouragements, and relentless supports. 


\section{TABLE OF CONTENTS}

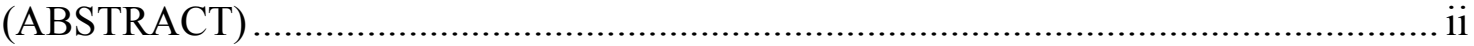

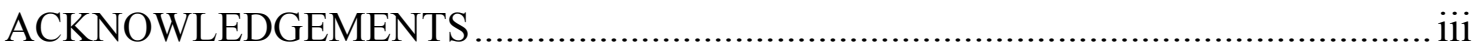

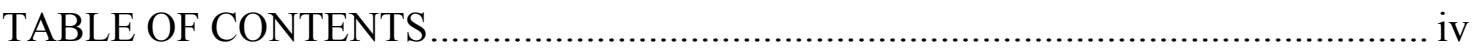

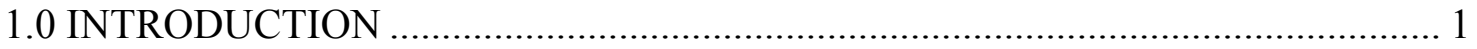

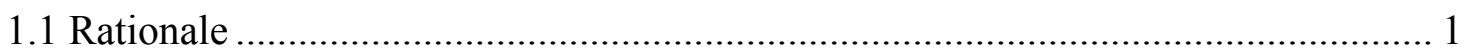

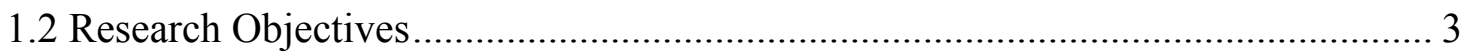

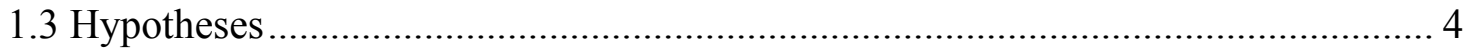

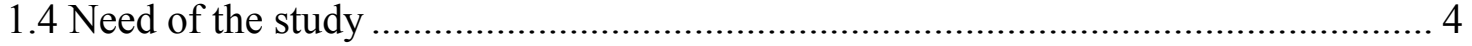

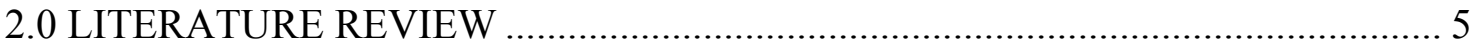

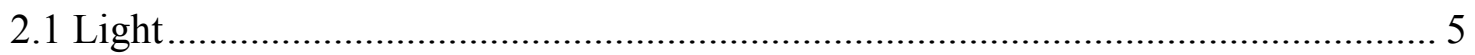

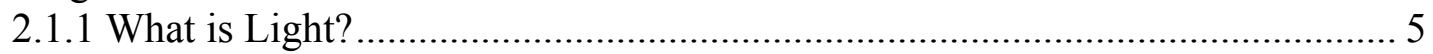

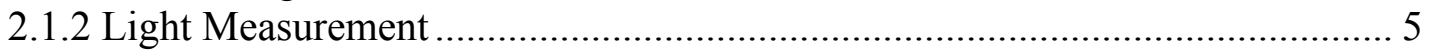

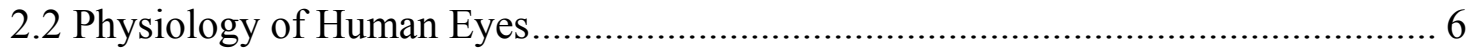

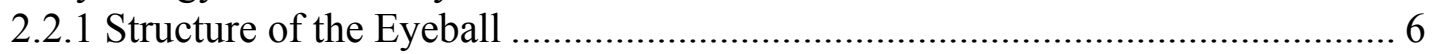

2.2.2 Mechanism of Scattering Light in our Eyes ................................................. 9

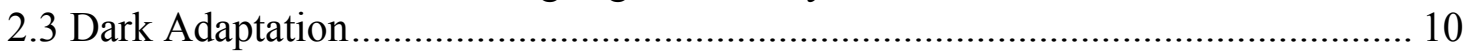

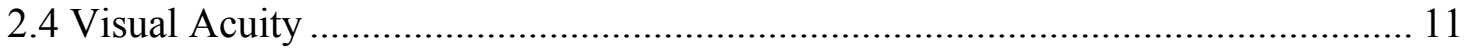

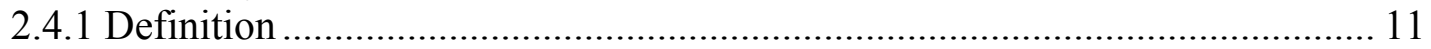

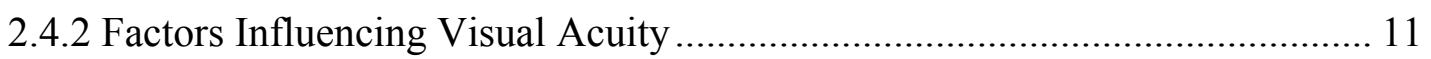

2.4.3 Measuring Methods ............................................................................... 12

2.4.4 Visual Acuity and Driving Performance........................................................ 17

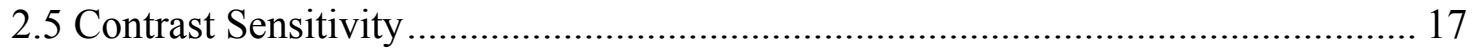

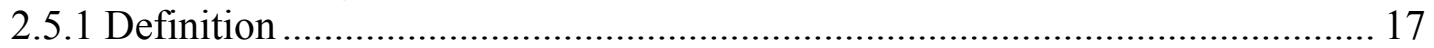

2.5.2 Factors Influencing Contrast Sensitivity.................................................... 18

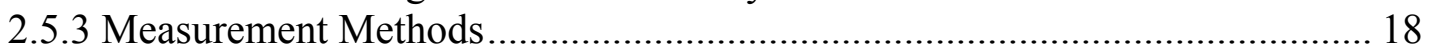

2.5.4 Contrast Sensitivity vs. Visual Acuity ………………................................ 21

2.5.5 Contrast Sensitivity and Driving Performance ………................................. 21

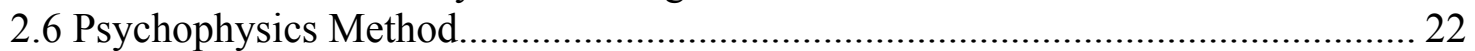

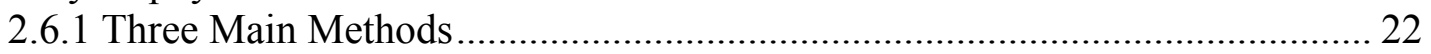

2.6.2 Controlling Bias and Guessing Responses ................................................... 23

2.7 Glare

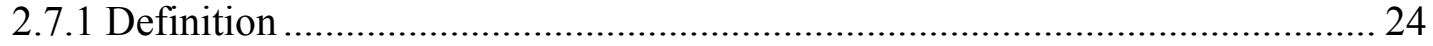

2.7.2 Physiology in Glare Exposure ………………......................................... 25

2.7.3 Factors Influencing the Level of Glare ......................................................... 25

2.7.4 Measuring method ............................................................................... 26

2.7.5 Glare and Driving Performance ………………........................................ 29

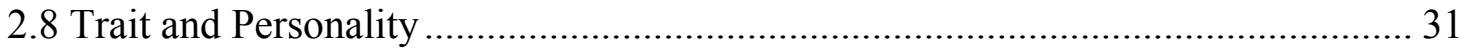

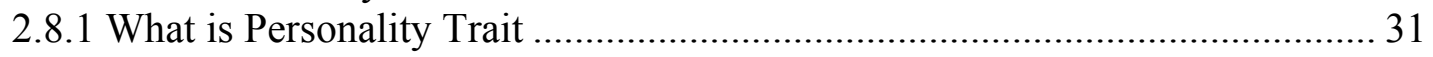

2.8.2 Growth and Development Effects on Personality........................................... 31

2.8.3 Five-Factor Model of Personality .................................................................. 32

2.8.4 Personality Assessment via Questionnaire .................................................... 35 
2.8.5 Individual Differences and Driving Performance............................................ 36

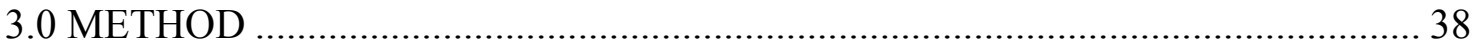

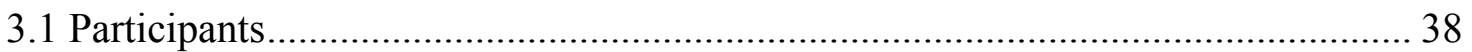

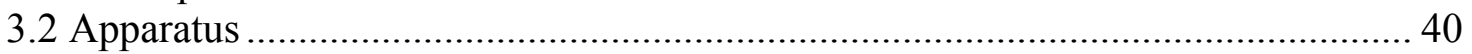

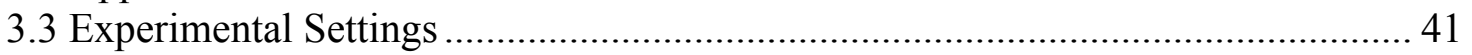

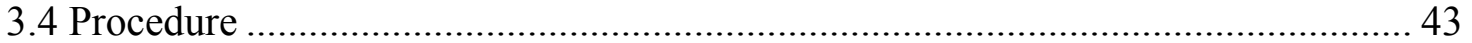

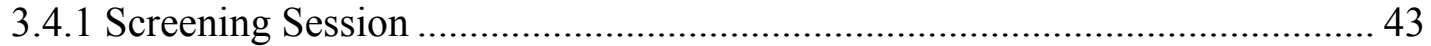

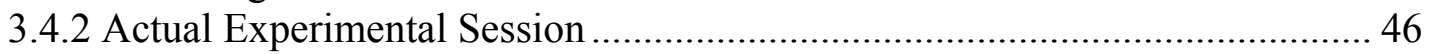

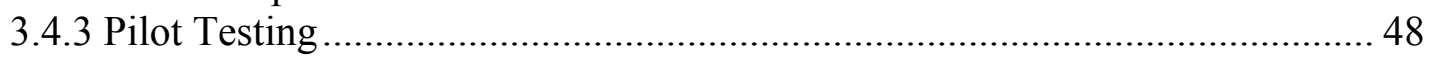

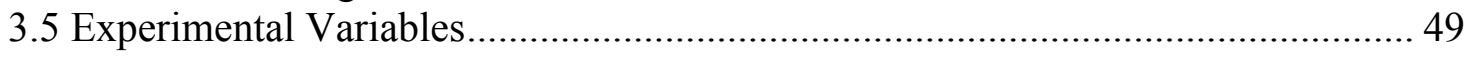

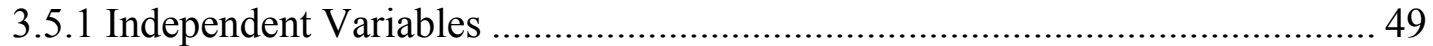

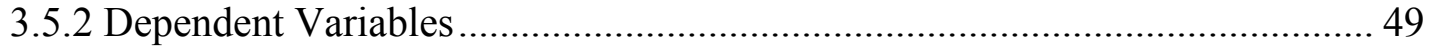

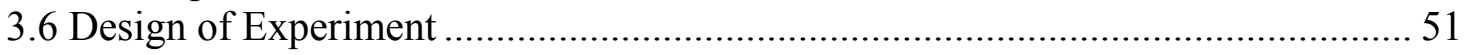

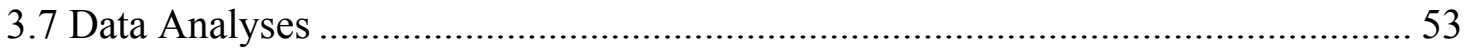

3.7.1 Multivariate Test:_................................................................................. 53

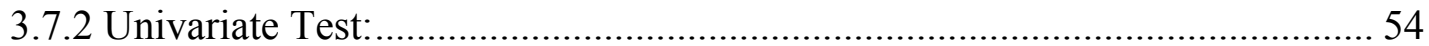

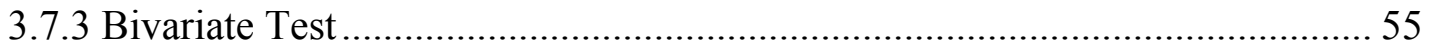

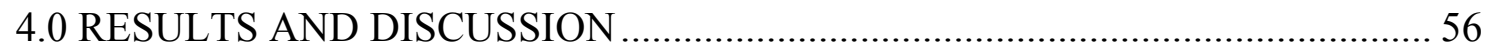

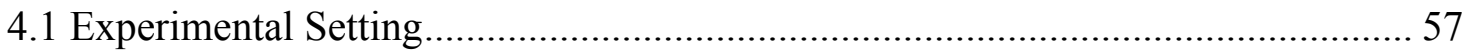

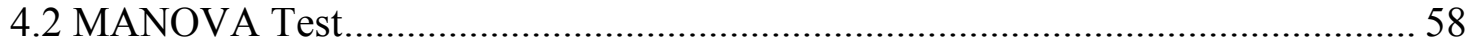

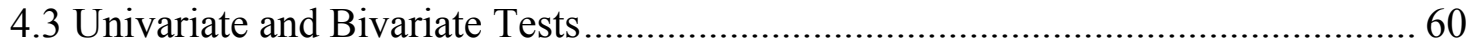

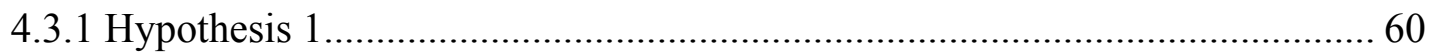

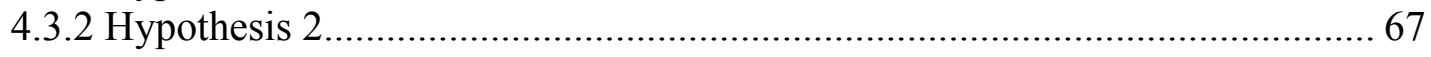

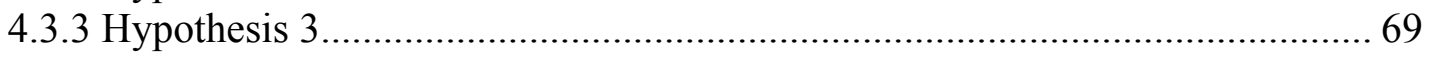

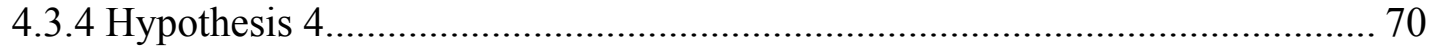

4.3.5 Hypothesis 5

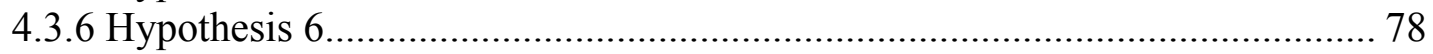

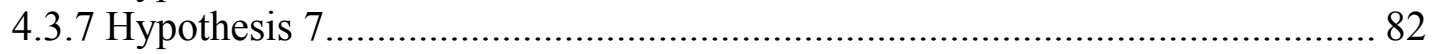

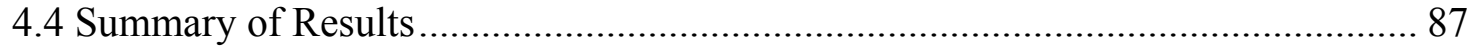

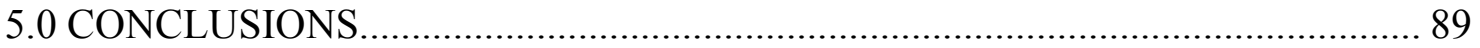

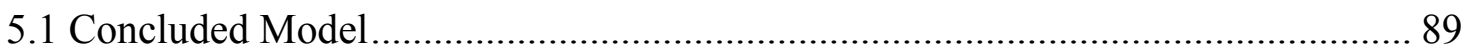

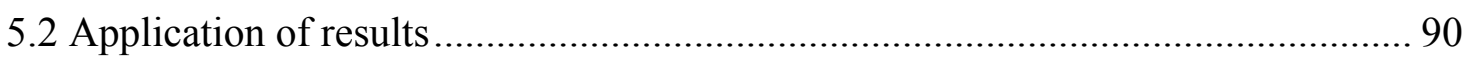

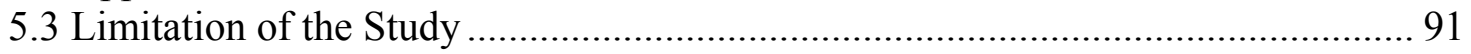

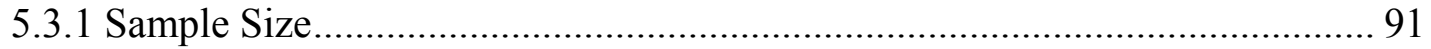

5.3.2 Reliability of Questionnaire Response......................................................... 92

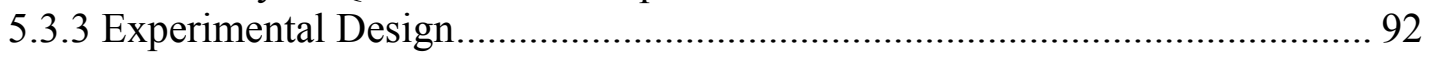

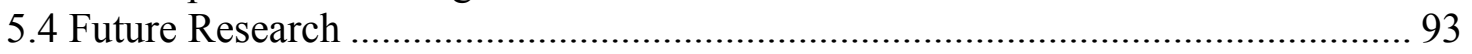

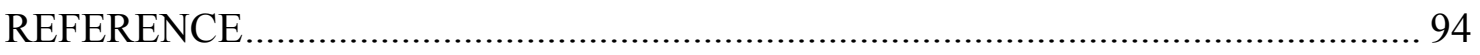

APPENDIX A: SCREENING TEST RESULTS ……………………................... 103

APPENDIX B: SECONDARY RESULTS AND DISCUSSION …………………....... 107

B1. Relationship between Screening and Actual Performance .............................. 107

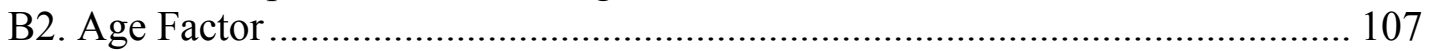

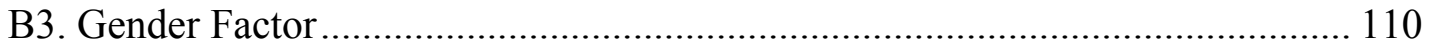


B4. Parsing Model (Partial out the Age and Personality Factors) ........................ 112

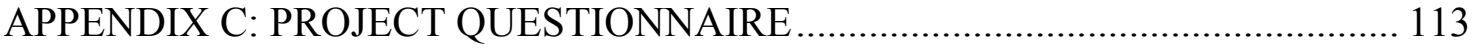

APPENDIX D: LANDOLT'S C-CIRCLE CHART ............................................... 115

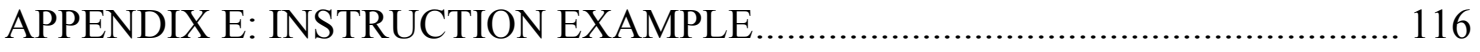

APPENDIX F: DISCOMFORT GLARE RATING SCALE .................................. 118

APPENDIX G: INFORMED CONSENT FORM .................................................. 119

Vita: Haruetai Mekaroonreung ......................................................................... 122

\section{Table of Illustration}

Table 1: Example of Observer's Responses in Method of Limit ................................... 23

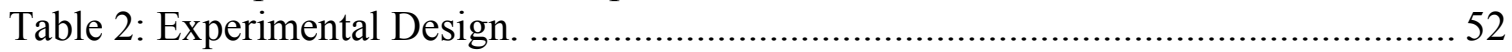

Table 3: Counterbalancing among Three Glare Simulated Levels ................................. 52

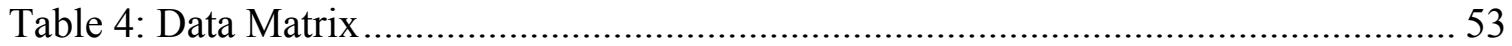

Table 5: Descriptive ANOVA Summary Table for Experimental Setting ...................... 57

Table 6: Personality Type Main Effect Test on CSI and Rating ...................................5 58

Table 7: Glare Level Main Effect Test on CSI and Rating........................................... 59

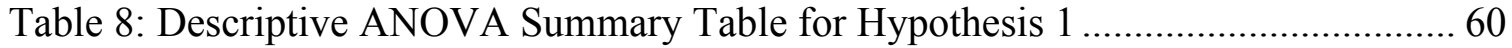

Table 9: Bivariate Regression Analysis Summary Table for Hypothesis 1..................... 60

Table 10: Descriptive Summary of CSI on Glare Level Main Effect - N Data ............... 61

Table 11: Analysis of Variance for Significant Linear Relationship between Measured

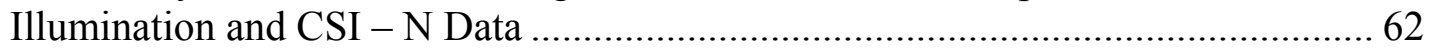

Table 12: Descriptive Summary of CSI on Glare Level Main Effect - E Data................ 62

Table 13: Analysis of Variance for Significant Linear Relationship between Measured

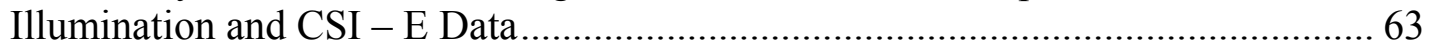

Table 14: Descriptive Summary of CS on Glare Level Main Effect - N Data ............... 64

Table 15: Analysis of Variance for Significant Linear Relationship between Rating and

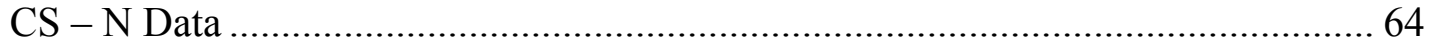

Table 16: Descriptive Summary of CS on Glare Level Main Effect - E Data................. 65

Table 17: Analysis of Variance for Significant Linear Relationship between Illumination

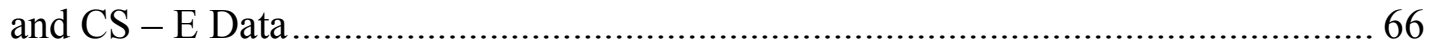

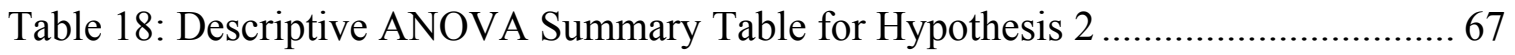

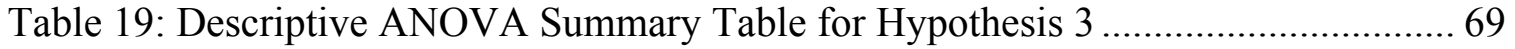

Table 20: Descriptive ANOVA Summary Table for Hypothesis 4 ................................ 70

Table 21: Bivariate Regression Analysis Summary Table for Hypothesis 4................... 70

Table 22: Descriptive Summary of Rating on Glare Level Main Effect - N Data........... 70

Table 23: Analysis of Variance for Significant Linear Relationship between Measured

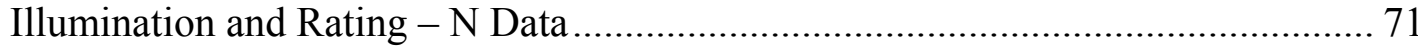

Table 24: Descriptive Summary of Rating on Glare Level Main Effect - E Data ........... 72

Table 25: Analysis of Variance for Significant Linear Relationship between Measured

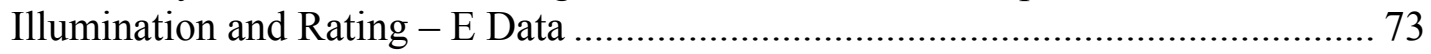

Table 26: Descriptive ANOVA Summary Table for Hypothesis 5 .............................. 74 Table 27: Bivariate Regression Analysis Summary Table for Hypothesis 5.................. 74 
Table 28: Analysis of Variance for Significant Linear Relationship between Neuroticism

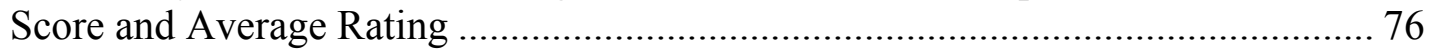

Table 29: Descriptive ANOVA Summary Table for Hypothesis 6 .............................. 78

Table 30: Bivariate Regression Analysis Summary Table for Hypothesis 6................... 78

Table 31: Descriptive Summary of Rating on Extraversion Type Main Effect .............. 79

Table 32: Analysis of Variance for significant linear relationship .............................. 80

Table 33: Bivariate Regression Analysis Summary Table for Hypothesis 7.................. 82

Table 34: Analysis of Variance for Significant Linear Relationship between Rating and CSI - N Data ...................................................................................... 82

Table 35: Analysis of Variance for Significant Linear Relationship between Rating and CSI - E Data ................................................................................. 83

Table 36: Analysis of Variance for Significant Linear Relationship between Rating and

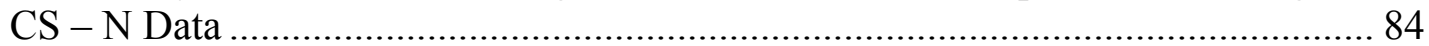

Table 37: Analysis of Variance for Significant Linear Relationship between Rating and CS - E Data.

Table 38: Participants Pool for Extreme Score on either Neuroticism or Extraversion Trait

Table 39: Participants Pool for Extreme Score on Both Neuroticism and Extraversion

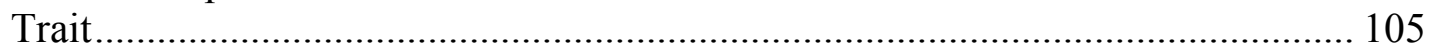

Table 40: Demographic Data of Participants included in the Study............................. 105

Table 41: Descriptive T-Test Summary Table for Vision Screening Results ................ 106

Table 42: Bivariate Regression Analysis between Screening and Actual Performance. 107

Table 43: Descriptive ANOVA and T-Test Summary Table for Age Factor................ 108

Table 44: Descriptive Summary of Rating on Gender Main Effect ............................. 108

Table 45: Descriptive Summary of Screening CS on Gender Main Effect ................... 109

Table 46: Descriptive Summary of CS on Age Main Effect ....................................... 109

Table 47: Descriptive ANOVA Summary Table for Gender Factor ............................. 110

Table 48: Descriptive Summary of CSI on Gender Main Effect................................... 110

Table 49: Descriptive Summary of Rating on Gender Main Effect ............................. 111

Table 50: Bivariate Analysis Using Parsing Data...................................................... 112

Figure 1: Proposed Model of Discomfort Glare Subjective Experience and Visual

Performance in Glare Situation............................................................................... 3

Figure 2: Horizontal Cross Section of the Human Eye.................................................. 7

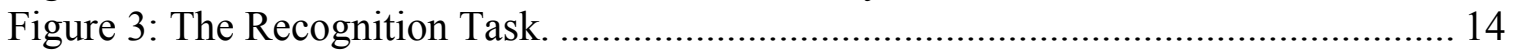

Figure 4: Dimension Ratio of the Landolt's C-Circle. ............................................ 15

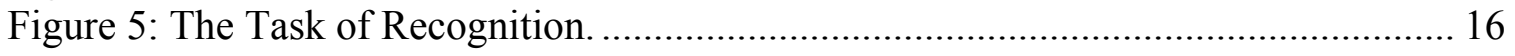

Figure 6: The Vernier Acuity Test................................................................................... 16

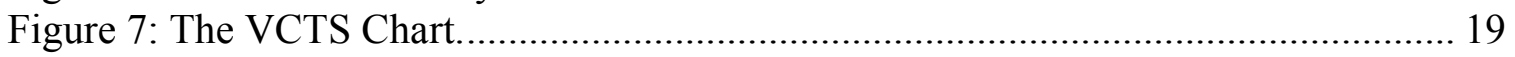

Figure 8: Experimental Settings for Visual Acuity Test.......................................... 42

Figure 9: Experimental Settings for Glare Test (top view)........................................ 43

Figure 10: Measured Area for Calculated Contrast Ratio .......................................... 50

Figure 11: Polynomial Third Degree Relationship between Dial Value and Calculated

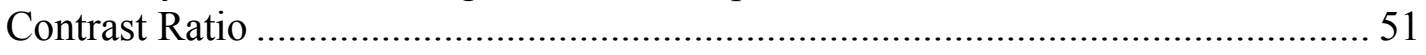

Figure 12: Personality Type Main Effect on CSI and Rating ........................................ 58

Figure 13: Personality Type Main Effect on CSI and Rating ...................................... 59 


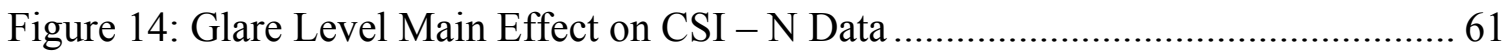

Figure 15: Scatter Plot between Measured Illuminance and CSI - N Data..................... 62

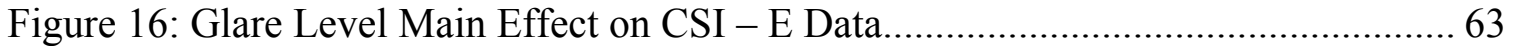

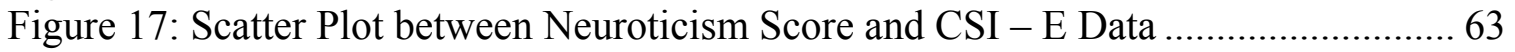

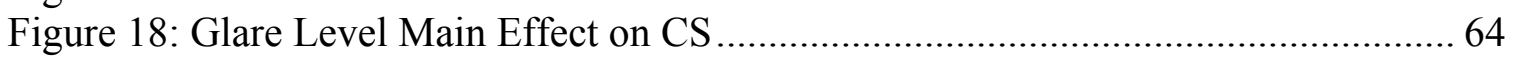

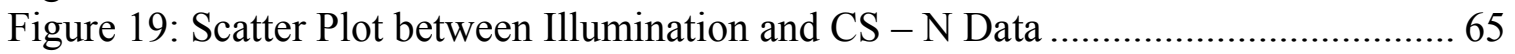

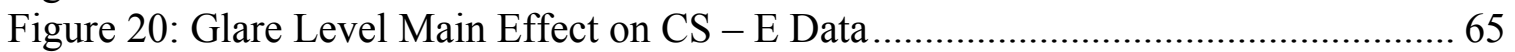

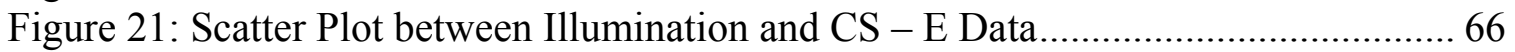

Figure 22: Glare Level Main Effect on Rating - N Data........................................... 71

Figure 23: Scatter Plot between Measured Illuminance and CSI - N Data..................... 71

Figure 24: Glare Level Main Effect on Rating - E Data ............................................. 72

Figure 25: Scatter Plot between Neuroticism Score and Average Rating - E Data ........ 73

Figure 26: Frequency Distribution of Average Rating Responses from High and Low

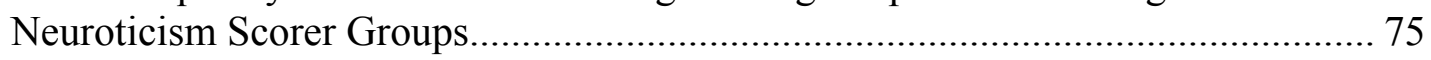

Figure 27: Scatter Plot between Neuroticism Score and Average Rating ....................... 76

Figure 28: Extraversion Type Main Effect on Rating .................................................. 79

Figure 29: Frequency Distribution of Average Rating from High and Low Extraversion

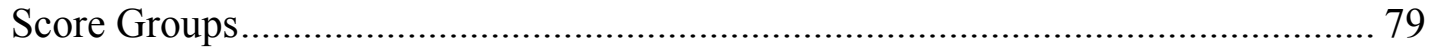

Figure 30: Scatter Plot between Extraversion Score and Average Rating...................... 80

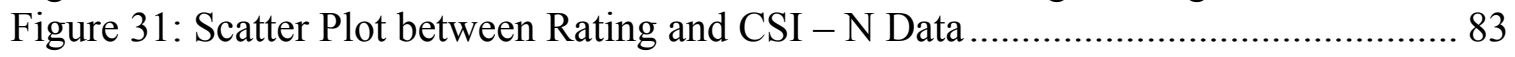

Figure 32: Scatter Plot between Rating and CSI - E Data ........................................... 84

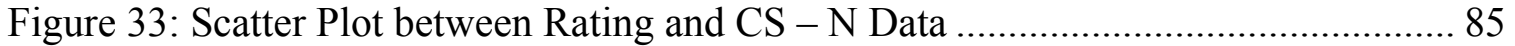

Figure 34: Scatter Plot between Rating and CS - E Data............................................. 86

Figure 35: Concluded Model of Discomfort Glare Subjective Experience and Visual

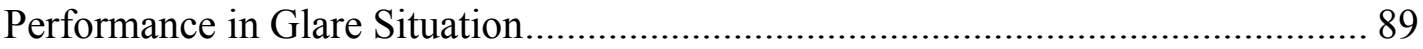

Figure 36: The Frequency Distribution of Neuroticism Scores.................................. 103

Figure 37: The Frequency Distribution of Extraversion Scores ..................................... 104

Figure 38: Age Main Effect on Visual Acuity............................................................ 108

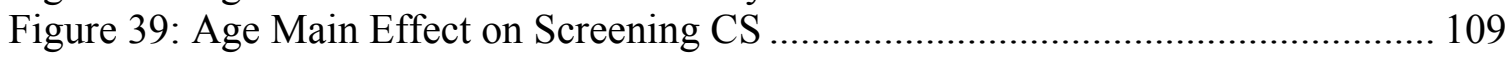

Figure 40: Age Main Effect on CS ............................................................................. 109

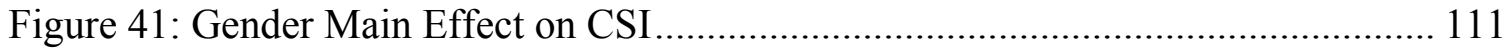

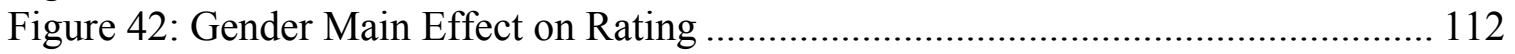




\subsection{INTRODUCTION}

\subsection{Rationale}

Feelings of discomfort glare have been widely assessed with rating scales for various purposes, especially in the design of lighting configurations such as street lighting and visual display terminal (VDT) systems. However, consensus is lacking among researchers in terms of discomfort glare assessment methods (Gellatly and Weintraub, 1990; Lennie \& Van Hemel, 2002; Olson \& Sivak, 1984; Sivak \& Flannagan, 1994). Although the between-subject variations in rating scores are repeatedly reported to be quite high, the subjective glare rating has been the method used for many years. Several subjective rating scales have been proposed since the 1960s (De Boer, 1967a). Among these rating scales, the 9 point De Boer scale is most often used in the field of automotive and public lighting systems (Theeuwes, 2002) while some other scales such as Visual Analog Scale (VAS) are used less frequently.

As suggested by few researchers (Berman et al., 1994; Theeuwes, 2002), one underlying reason for the large variability of this subjective response could be the result of differences in the observers' trait rather than visual impairment. For example, people with dispositions related to worry or anxiety may have a perceived criterion for discomfort that is much lower than those who have dispositions that are less anxiety or worry prone. As a result, the worried people could exhibit more discomfort. In other words, the variability may have been due partly to the trait-based individual difference factors rather than the physiology factors (i.e., visual impairment).

Support for the above statement comes from the studies relating discomfort glare feelings with visual-related task performances (Sivak et al., 1991; Theeuwes, 2002). Theeuwes (2002) compared actual driving performance with subjective glare experiences and found that the relationship between the two variables was low. Similarly, Sivak et al. (1991) found low correlation between detection performance and discomfort glare rating response. Furthermore, contradiction on the relationship between age and glare discomfort rating (Petherbridge \& Hopkinson, 1950) may also be interpreted using similar suggestion (i.e., the aging participants did exhibit lower vision performance but 
were not significantly different from the younger group in terms of perceived discomfort).

According to the personality trait theory (the five factor model) suggested by McCrae \& Costa (1990), human personality can be categorized into 5 main dimensions, namely Neuroticism (N), Extraversion (E), Openness (O), Agreeableness (A), and Conscientiousness (C). For this particular study, only the first two dimensions are hypothesized as factors that could affect discomfort glare experience, as many researchers have found relationships between stimulus arousal level and extraversion personality score (Gilbert \& Hagen, 1985; Wigglesworth \& Smith, 1976). Similarly, a tendency to feel depressed and complain about things is found to be related with neuroticism personality score (McCrae \& Costa, 1991; Watson \& Clark, 1984). Moreover, another reason why this study concentrates on using these personality dimensions is that these two dimensions are found to be correlated to each other (i.e., the high extraversion scorers usually have low neuroticism scores) (Eysenck, 1982).

On the other hand, some other factors have been found to influence the discomfort glare feeling as well. These factors include light source characteristics (e.g., luminance level, light color characteristic, light source size), environmental settings (e.g., background luminance, angle between the observer's line of sight and the direction of the light source), and external elements (e.g., difficulty of task currently being performed, past experience on glare exposure). For this study, only glare intensity was used to manipulate the glare levels while the other factors were controlled as much as possible.

Besides the psychological changes, adverse effects under glare situations are also found in visual performance. Among these effects, a lowering of observer's contrast sensitivity is widely agreed to be a consequence of glare exposure resulting from the scattered light in the observer's eyes (e.g., Miller \& Benedek, 1973). Therfeore, the contrast sensitivity index (CSI), calculated from the difference between contrast sensitivity threshold in no glare condition and the one in glare condition, was used in this current study as the measure of actual performance.

In conclusion, the proposed model of this study is shown in the following diagram (Figure 1). 


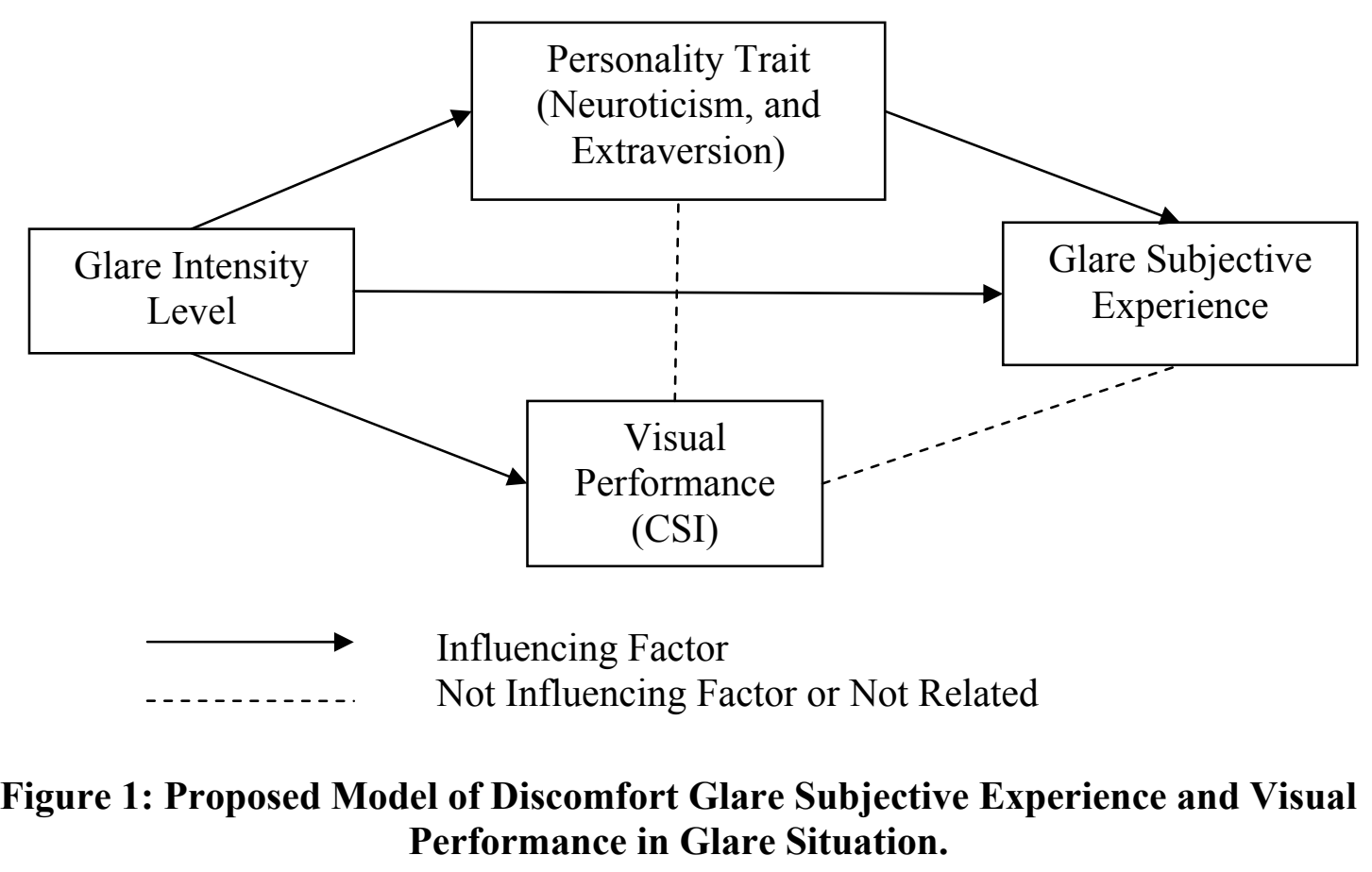

In referring to the proposed model, glare subjective experience is affected by both glare intensity level and trait-based individual differences while visual performance is directly related to only glare intensity level. Thus, subjective experience and visual performance is hypothesized to have a low correlation. Note that the low relationship between actual performance and personality trait is hypothesized as a result of screening tests introduced in this study. According to Linnie \& Van Hemel (2002), the adverse effect on contrast sensitivity from glare exposure is dependent on visual performance in no glare condition. Thus, since all participants were required to pass two defined visual tests (i.e., visual acuity and contrast sensitivity), they would not be expected to gain similar adverse effects from glare exposure as measured by CSI.

\subsection{Research Objectives}

The objectives of the proposed study are:

1. To investigate the effect of trait-based individual differences (neuroticism and extraversion) on glare subjective experiences and contrast sensitivity performance when exposed to the same manipulated glare condition. 
2. To investigate the correlation between the glare subjective experiences and actual contrast sensitivity.

\subsection{Hypotheses}

1 As the intensity of glare source increases, the contrast sensitivity index (CSI) will also increase (the visual contrast sensitivity will decrease).

2 Participants with different neuroticism scores will have no significant difference in contrast sensitivity index (CSI).

3 Participants with different extraversion scores will have no significant difference in contrast sensitivity index (CSI).

4 As the intensity of glare source increases, the subjective glare experience (as measured by modified De Boer scale) will also increase.

5 Participants with high neuroticism scores will report higher subjective glare experience (as measured by modified De Boer scale) compared to people with low neuroticism scores.

6 Participants with high extraversion scores will report lower subjective glare experiences (as measured by modified De Boer scale) compared to people with low extraversion scores.

7 Subjective glare experience (as measured by modified De Boer scale) will have low relationship with the measured contrast sensitivity index.

\subsection{Need of the study}

1. One benefit of the proposed study is directed toward improving our understanding of influencing factors on discomfort glare experience which may eventually be applied to the design of glare measurement methods.

2. Application of results can improve training and selection of drivers and workers who may work under conditions of glare. 


\subsection{LITERATURE REVIEW}

This literature review is divided into seven main sections. The first section discusses the basics of light and light measurement units that will be repeatedly used in the review. The second section then presents physiology of human eyes; the structure of the human eye, and mechanism of scattering light that occurs in glare situations. Dark adaptation is elaborated in the third section. The next two sections discuss the important visual performance information that will be utilized in the study; namely visual acuity and contrast sensitivity. The sections are presented with elaborate information on the definition, influencing factors, measuring method, and relationship to driving performance. The sixth section presents the psychophysics method that will be utilized in the study. Glare related issues are included in the seventh section. Finally, trait-based individual differences utilizing the five-factor model are discussed.

\subsection{Light}

\subsubsection{What is Light?}

Light is a portion of the electromagnetic spectrum that produces a visual response (Stanley et al., 1979). This visible light ranges from $380 \mathrm{~nm}$ to $770 \mathrm{~nm}$. This portion is considered very small as compared to all existing spectra of electromagnetic waves.

\subsubsection{Light Measurement}

In order to standardize light measurements, photometric units were invented to describe the vision stimulus or light based on a standard candle. The light property can be measured in various ways. Some of the most common quantities are listed and described as follows (adapted from Stanley et al., 1979):

1. Radiance: The amount of energy coming from a light source measured in "Lumen."

2. Luminance: The amount of light reflected from a surface measured in "Candela per meter square." 
3. Illuminance: The amount of light falling upon a surface measured in "Lux."

4. Reflectance: The proportion of light reflected from surface calculated in percent of luminance as compared to illuminance values.

5. Brightness: The subjective sensation of visible light. Note that no unit is currently agreed to use for brightness measurement but "bril" is currently considered the best to use.

\subsection{Physiology of Human Eyes}

\subsubsection{Structure of the Eyeball}

Some main structures of the human eye are discussed in the following section. The horizontal cross section of a human eye is illustrated in Figure 2.

\section{$\underline{\text { Cornea }}$}

The cornea is a tough and transparent component of the eye located at the most anterior surface. No blood vessels are present in this special tissue (Cogan \& Kinsey, 1942). The center of the cornea is relatively thinner than the area at the periphery $(0.8$ $\mathrm{mm}$. vs. $1.0 \mathrm{~mm}$.). Approximately $90 \%$ of its thickness is composed of water and collagen. The cornea is one of the most sensitive components in our eyes. We can sense pressure, touch, and temperature through this tissue. It is the cornea curvature that is responsible for up to two-thirds of the total eye optical power (the index of refraction between the cornea and the external air is very high), while other components account for only one-third (e.g., interface between aqueous humor and cornea, interface between aqueous humor and crystalline lens).

\section{Intraocular Fluid (Aqueous Humor)}

In order to maintain the shape of the human eye, the "aqueous humor" (fluid-like substance) fills the chamber between the cornea and the crystalline lens. Its composition is very similar to that of blood plasma, but with a lower protein concentration. This fluid is continually produced and exuded from the chamber to preserve the range of intraocular pressure between 15 and $18 \mathrm{~mm}$. Hg. higher than the surrounding pressure (Troncoso, 
1942). In addition, upholding this chamber area also benefit the constant corrective refraction index in our eyes.

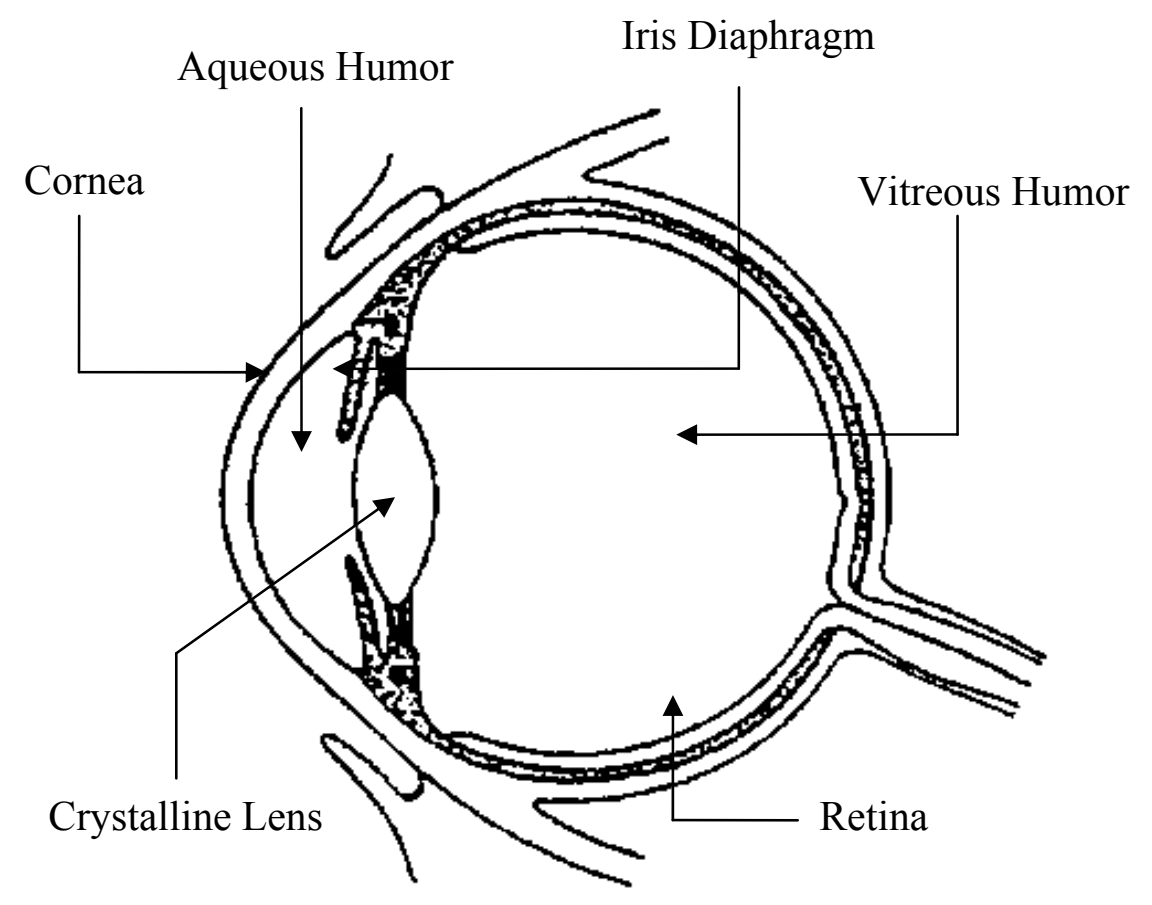

Figure 2: Horizontal Cross Section of the Human Eye.

\section{$\underline{\text { Iris Diaphragm }}$}

The iris diaphragm is located in the aqueous humor chamber. The front of this diaphragm contains the visible pigment of the eye, whereas the back contains black pigment. The most important function of the iris is to control the amount of light entering the crystalline lens, a transparent tissue behind the diaphragm. The central opening of the iris is called the "pupil". The pupil can be adjusted to various diameters from approximately 2 to $8 \mathrm{~mm}$. (Davson, 1949; Wagman \& Nathanson, 1942). The adjusted process is due to either contracted or dilated activity of the specialized smooth muscles bond with the pupil. As pupil size changes, the quality of image formed on the retina can be refined. Under normal conditions, the pupils of both eyes are about the same size even where they are exposed to different illuminance levels. 


\section{The Crystalline Lens}

The crystalline lens is an elastic transparent structure having neither blood nor nerve vessels. Major lens nutrition comes from the fluid of the aqueous humor. The lens itself is mainly composed of water and fat with some degree of protein. This protein content is used to absorb the infrared and ultraviolet light that could damage our lens. Although the contribution of the lens to the total visual ability is relatively small, changes in its shape afford some adjustment of the ability and permit variations of focus for objects at different distances. Suspended by smooth muscles, the lens shape is adaptable according to these muscles' status. In the resting condition, the lens will remain flat and our eyes will function properly for focusing at relatively distant objects. However, to look at closer objects, the muscles will re-adjust so that the lens becomes thicker and images of near objects will be focused correctly on the retina. People with normal eyes will have the ability to focus at near objects from 4 or 8 inches to 20 feet (Duane, 1922). Note that accommodation to near objects occurs much more rapidly than far objects.

In terms of aging eyes, the yellow pigmentation in our lens will naturally increase (McEwen, 1959). The lens will tend to lose water and become hardened, which eventually results in lower capability to accommodate lens thickness.

Forward light scatter from lenticular changes in the elderly human eye can be simulated by dabbing petroleum jelly onto a spectacle lens surface. The characteristic at scattering of a lens prepared in this way is similar to that of a human cataract.

Additionally, an optical cell containing polystyrene microspheres in a water suspension can be used to simulate age-related lens changes. The conclusion from these two studies is that simulated lens opacities, which have little or no effect on Snellen acuity, can severely impair the directional discrimination of low contrast targets.

\section{$\underline{\text { Vitreous Humor }}$}

The posterior compartment of the eyeball contains a jelly-like fluid called "Vitreous Humor." It occupies up to $80 \%$ of the eyeball. In order for the light to reach the retina area, it will have to pass through this vitreous humor. Virtually no light distortion is further introduced in this area. 


\section{$\underline{\text { Retina }}$}

The retina is the innermost membrane in our eyes. It lies along the posterior area of the eyeball. The retina is composed of approximately ten layers of tissue. However, all tissues are grouped into three main layers, namely photoreceptors, bipolar cells, and ganglion cells (ordering from innermost to outermost area). Some blood vessels are found in the middle layers of the retina. Therefore, the light that travels to reach the photoreceptors will be required to pass through all those blood vessels. Rods and cones are special organs found in the photoreceptor area that are used for image perception. Rods are normally responsible for sensing any moving targets and are found mostly at the area away from the center of the back of the eyeball. On the other hand, cones, which are mostly found at the center of retina, are heavily used for acuity perception.

In the retina, all the nerves join together and enter the brain through the back of the eye. There are no rods and cones at the spot of nerve connection. This missing area is known as the "blind spot." No image will be processed if the reflected light from an object lies in this particular area.

\subsubsection{Mechanism of Scattering Light in our Eyes}

Normally, visual performance will be better with light. However, with excessive light or interfering light sources, our ability to perceive an object deteriorates. For example, direct light on the fovea region of our retina will cause our retina-brain combination to become unable to discriminate the image in the visual field (the image which would be perceived without this extra light source).

This off-axis light is scattered by the ocular media onto our fovea image (the "E" letter in this case) on the retina and further reduces discrimination of the target " $E$ " from its background. Please refer to Miller \& Benedek (1973) for illustration. Since the light scattering mechanism is altered according to the light source position, this scattering effect can be considerably reduced by moving the light source to further angles (larger angle from the observer's line of sight) or by lowering the light intensity itself. The quantitative explanation of this phenomenon can be found in earlier studies (e.g., Holladay, 1926). 


\subsection{Dark Adaptation}

Having the ability to adapt its sensitivity, our eyes can function properly under a range of different ambient illumination levels. Although adaptation automatically occurs, it is not an abrupt response, especially when dealing with adaptation to darkness.

Through the use of psychophysical methods, it has been found that our eyes require about a twenty-minute period for adapting to darkness (Dark Adaptation) while only a few seconds are needed to adapt to a bright environment (Bright Adaptation). Interestingly, these human light adaptation processes are correlated well with natural activities. In nature, the transition from light to dark, which occurs at twilight, also takes about 20 minutes, whereas the adaptation from dark (sleeping time) to bright (wake up time) needs to be completed in seconds (Stanley et al., 1979).

The drop in threshold is a commonly measured variable used by researchers to study the dark adaptation process. The experiment starts by moving participants from a normal bright environment into a dim room. Then, their absolute thresholds are measured at different time intervals, and plotted into a curve. Using this method, there is a possibility that the dark adaptation process depends mainly on the retina's responses to the light. In the beginning (approximately first 10 minutes), the observers require very strong intensity to detect the stimuli. This first part of adaptation involves only the cones. Then, another similar adaptation period continues and the rods are dominantly used. In this second period, we will rapidly adapt and reach the stabilized level at about 10 minutes. Note that the kink in the sensitivity curve indicates a change in the rate of dark adaptation.

Study of the structural changes of the retina under the influence of light and darkness has further supported the concept of utilizing rods under dim light. It was found that rods contracted in darkness and elongated under the influence of light while cones behaved in the opposite manner (Jayle et al., 1959). This shows that rods are protected from strong light and thus, are used in dim light. When raising questions about age related effects on dark adaptation, research results are still contradictory. While some studies show that the rate of dark adaptation is slower for older people (Domey, McFarland, \& Chadwick, 1960; McFarland 1968; McFarland, Domey, Warren, \& Ward, 
1960), other studies demonstrate no significant differences between young and old (Eisner, Fleming, Klien, \& Mauldin, 1987).

\subsection{Visual Acuity}

\subsubsection{Definition}

Visual Acuity refers to the human ability to discriminate the fine details of a visual stimulus located in the field of view (Riggs, 1965). It is usually specified by critical dimensions of the testing object identified correctly (e.g., the stimulus size, approximate retina image, or visual angle). Generally, the tested person is presented with a group of objects and required to give a response about some critically fine details of the smallest object that they can still detect. Several of the widely used measurement methods are discussed in the following sections.

\subsubsection{Factors Influencing Visual Acuity}

Many factors are known to affect our visual acuity. This particular section is discusses the important factors influencing this visual performance.

\section{Retina Localization of the Image}

In 1957, Polyak found the relationship between visual acuity and number of cone receptors. He showed that the inner fovea, where our acuity reaches its highest, is also the region where the cones are predominant. Thus, when an image is transferred into the eye fovea region, our ability to see that object will stay very high. Then, as the image falls farther away from the fovea region, and the number of cones decreases, visual acuity will become worse. Of even greater importance is the fact that only in this fovea region does the ratio of optic nerve fibers to cone receptors reach one-to-one relation whereas further away to the periphery, the ratio will be much higher.

\section{Ambient Brightness}

As mentioned in earlier section, in the low ambient light environment, the rod receptors will react to the light while cone receptors will be protected. Using only rod 
receptors, our visual acuity will worsen (Jayle, 1959). This fact is supported by a study (Corso, 1981), which has found that for all ages, acuity improves when the overall illumination is increased. Therefore, anything that affects perception of brightness (e.g., the adaptive stage, actual ambient illuminance level) will also influence our visual acuity. $\underline{\text { Pupil Size }}$

Often compared to the camera lens, pupil size is a factor regulating the amount of light that passes through our eyes to reach the receptors. When the pupil becomes larger, more light can be reached at the retinal layer. This increase in received light results in more stimulated receptors and reduction of the blur due to light diffraction. On the other hand, when the pupil becomes smaller, less light can get into our eyes and hence, account for minimizing aberrations. Realizing two opposite influences of pupil size, it is believed that our eyes automatically adjust to gain the optimal level of visual acuity for any circumstance depending on luminance level, size of the seen object, and individual differences. For example, in dim light, the visual acuity ability becomes less important than the increased sensitivity obtained by increasing the amount of light entering the eye; thus, the pupil size increases to let in more light (Duke-Elder, 1963).

Age

Due to physiological changes (e.g., reduced pupil size, increased absorption by the ocular media, increased light scatter), aging adults are found to have significantly worse visual performance including visual acuity. It is found that at the age of 20 to 60 years, individuals would be more likely to have better than visual acuity of 20/20 and as people age loss of vision will dramatically increase. The very common impairment for older adults is farsighted eye vision. The decline generally begins after age 40 or 50 and continues throughout the rest of life (Weymouth et al., 1928). By age 75, the acuity declines to about 0.6 or $20 / 30$ (Pitts, 1982) and to about 0.35 or 20/60 for 90 -year-olds.

\subsubsection{Measuring Methods}

Many researchers have adopted different techniques to assess visual acuity. Regardless of methods, the National Research Council (National Research Council, 1994) has recommended lighting for acuity testing to be approximately at $160 \mathrm{~cd} / \mathrm{m}^{2}$ and with minimum glare. 
The following discussions are devoted to the explanation of four different acuity testing methods. Note that, due to the availability of testing equipment, this proposed study has adopted the recognition acuity testing system, known as "Landolt's C-Circle".

\section{Detection Acuity}

This measurement method is very simple. It requires the participant to respond whether a predefined object is present or not present in the visual field of view. The reciprocal of the angle in minutes subtended at the eye of the smallest detectable object will then be used as the measure of visual acuity. In this test method, given objects are normally in various levels of contrast as measured between an object and its background.

\section{$\underline{\text { Recognition Acuity }}$}

This tool is most commonly used in ophthalmologists' offices. It, sometimes, is referred to the "Eye Chart." For this system, visual acuity measure is given relative to the visual acuity of "normal" population. For example, 6/6 means that a person can identify the same letter at 6 meters as those with normal vision would be able to at 6 meters (or equivalently $20 / 20$ in feet). On the other hand, a visual acuity of 6/9 refers to someone who has lower ability. In this latter case, the person will be able to identify a letter at 6 meters that a normal person could view from 9 meters.

According to Lennie \& Van Hemel (2002), visual acuity at 20/200 or worse that persists after best correction is considered blind under the Social Security Administration (SSA) guidelines. This standard appears to have resulted from a previous study done by Snell (1925), who found that the "threshold for incapacity" fell between 20/200 and 20/400. Two well-known charts that fall in this testing category are the Snellen Acuity Test and the Landolt's C-Circle shown in Figure 3. The standardized letters of the alphabet introduced by Snellen were introduced in 1862 while the Landolt ring or square developed by Landolt was later proposed in 1889 in order to minimize the problems with literacy levels. 


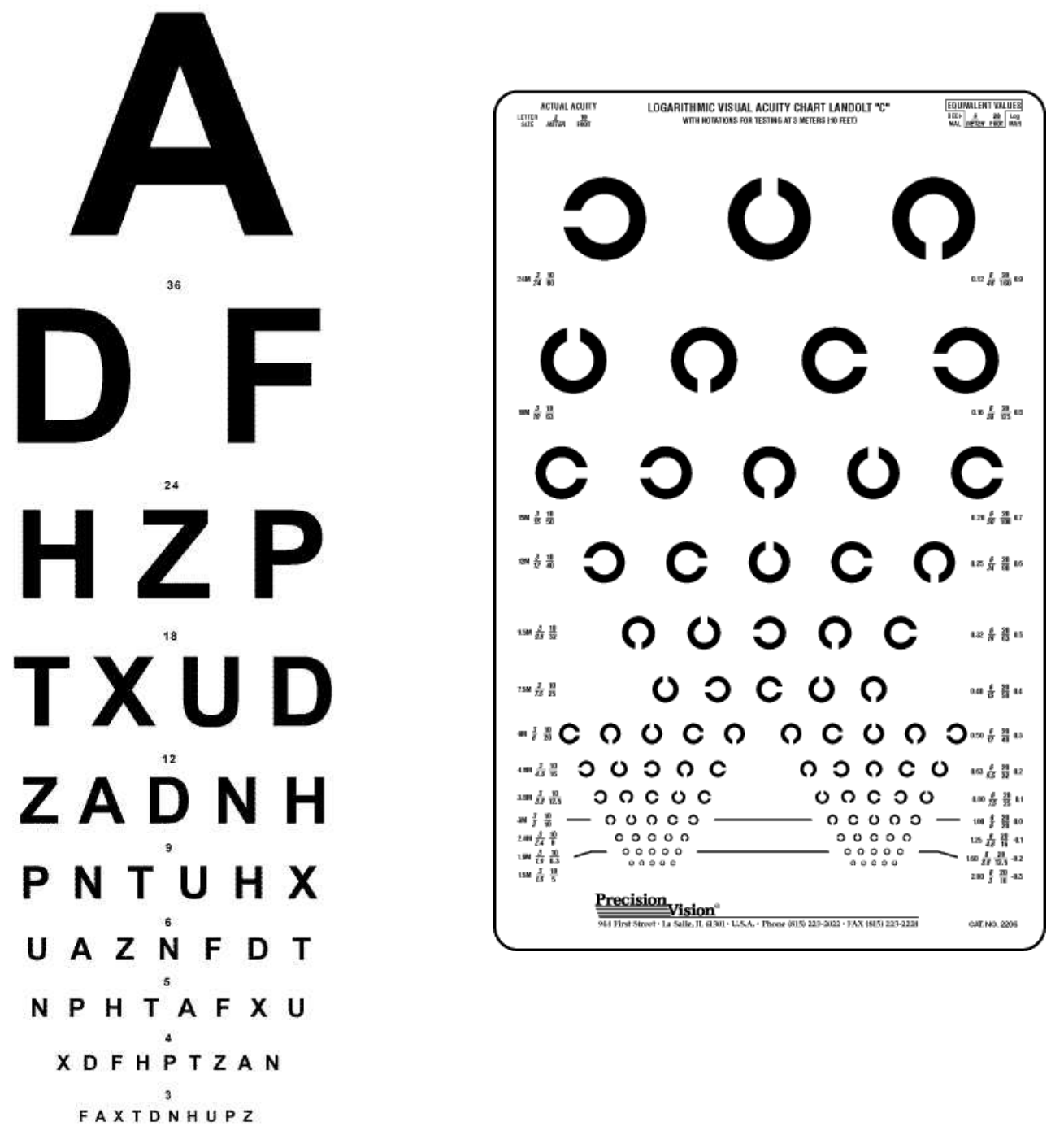

Figure 3: The Recognition Task.

(Left) Snellen Acuity Test, (Right) Landolt's Circles Test

1. In the Snellen Acuity Test, observers are required to look at a specially designed chart that shows a list of randomly predefined alphabets in progressive size order. Then, the observers are asked to read the smallest letter they can recognize from the list. Normal testing distance is set at 6 meters to allow observer's eye muscles to be in a state of rest. For this particular method, a low luminance testing method was developed called the "SKILL Card" (Haegerstrom-Portnoy et al., 1997). The card uses a dark gray acuity card 
with black letters in order to simulate stimuli under low-contrast, low-luminance conditions. The benefit of this card method is that the test can be done without actually turning down the lights and measuring light levels.

2. In the Landolt's C-Circle Test, observers are presented with a group of circles, each of which has a broken gap pointing either up, down, left, or right. Observers are required to identify the opening gap direction of the smallest circle they can see. As a rule, the Landolt C-Circle is designed using the 5:1:1 ratio, meaning the ratio of diameter of the circle, thickness, and length of the gap respectively (Figure 4). Visual acuity is measured by the visual angle of the smallest opening gap that can be resolved. For example, if the gap has a visual angle of A degree or $60 \mathrm{~A}$ minute, the visual acuity is determined by $1 / \mathrm{A}$ (the reciprocal of the angle of the gap). Therefore, if the angle is 1 minute, then visual acuity is defined as 1.0 .

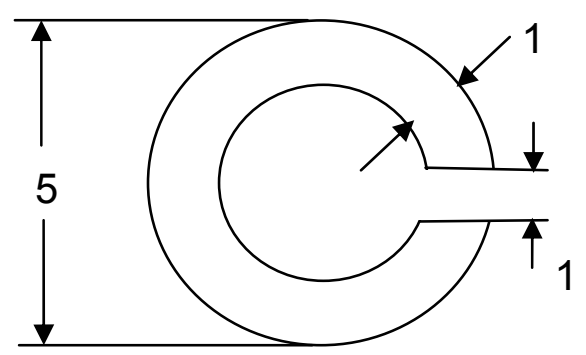

Figure 4: Dimension Ratio of the Landolt's C-Circle.

\section{Resolution Acuity}

The tests in this category are intended to find the minimal distance among object groups that can still be recognized as separate. Various patterns are used for this test as shown in Figure 5.

Basically, this method is based on the fact that increasing the number of single elements can further reduce our perceptual ability to perceive separateness. Therefore, the minimum acuity found with this test will be higher than with one testing a single element (e.g., grating composed of 20 black lines alternating with 20 white lines vs. 2 dark lines with 1 white line in between). Visual acuity, in the sense of resolution, is the reciprocal of 
the angular separation between two elements of the test pattern when the two images are barely resolved.
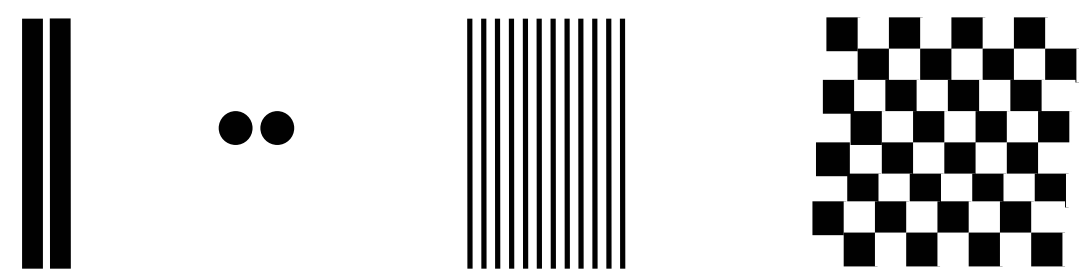

Figure 5: The Task of Recognition.

(From left to Right) Double line target, Double dot target, Acuity Grating, and Checkerboard.

\section{Localization Acuity}

Lastly, another useful acuity test classification is called "Localization Acuity Test." This acuity test refers to the small displacement of one element in the object as compared with another element and perceived as offset from one another. "Vernier acuity," shown in Figure 6, is a well-known test of this acuity type.

$$
1
$$

Figure 6: The Vernier Acuity Test.

This acuity is defined as the reciprocal of the angle subtended at the eye by a lateral displacement that is barely discriminated by the participant. Therefore, participant will be asked to identify whether the line is a broken line or a continual line while the amount of displacement among them is continually decreased. 


\subsubsection{Visual Acuity and Driving Performance}

Though driving a vehicle is shown to be a highly visual dependent task, a number of studies have shown that there is no clear relationship between visual acuity and driving performance (Ball et al., 1993; Decina \& Staplin, 1993; Gresset \& Meyer, 1994; Hills, 1980; Ivers et al., 1999; Johansson et al., 1996; Marottoli et al., 1994; McCloskey et al., 1994; Owsley et al., 1998; Owsley et al., 1991; Shinar \& Schieber, 1991). However, some other tasks such as reading road signs and avoiding obstacles in the roadway, are found to be affected by visual acuity loss (Higgins et al., 1998). In addition, it is important to point out that low visual acuity individuals are normally less exposed to driving tasks due to both state laws and self-restriction.

\subsection{Contrast Sensitivity}

\subsubsection{Definition}

According to Lennie \& Van Hemel, (2002), "contrast sensitivity is a measure of the lowest contrast that an observer can detect." To understand the contrast sensitivity, we will first need to define the term "contrast ratio." As expressed in Weber's Contrast Ratio, the contrast ratio is the ratio of difference between the maximum and minimum luminance and the maximum luminance itself. In other words, the quantitative expression of this value can be stated as:

$$
\begin{aligned}
& \text { Contrast Ratio }=(\mathrm{L} \max -\mathrm{L} \min ) / \mathrm{L} \max \\
& \text { Where: } \quad L \max =\text { Maximum Luminance }\left(\text { in } \mathrm{cd} / \mathrm{m}^{2}\right) \\
& \mathrm{L} \min =\text { Minimum Luminance }\left(\text { in } \mathrm{cd} / \mathrm{m}^{2}\right)
\end{aligned}
$$

Note that $\mathrm{L}$ max and $\mathrm{L}$ min are often the luminance levels of the detected object and the background luminance. Then, after the detectable lowest contrast level is tested, the contrast sensitivity is simply calculated as the reciprocal of that value. Thus, if a person can detect a very low contrast object (contrast ratio moving toward zero or when $\mathrm{L}$ max is very close to $\mathrm{L} \mathrm{min}$ ), his/her contrast sensitivity is considered high. 


\subsubsection{Factors Influencing Contrast Sensitivity}

In general, factors that affect visual acuity are also known to affect the contrast sensitivity level. However, decreasing performance with aging adults is found only at high spatial frequencies (Derefeldt et al., 1979; Kline et al., 1983; Madden \& Greene, 1987; Schieber et al., 1992).

\subsubsection{Measurement Methods}

\section{Computer Generated Gratings}

In the laboratory, contrast sensitivity is usually measured psychophysically. The observer is asked to respond when he or she first detects a pattern or when the pattern is no longer seen. Normally, patches of grating (bars) patterns that vary over a wide range of spatial frequencies are used. The gratings are computer generated and displayed on a computer screen or cathode ray tube (e.g., Cadwell CTS-5000, B-Vat, NIC Optronics CS2000 from Nicolet). This measurement method allows the experimenter to construct a contrast sensitivity function. However, this type of laboratory test requires sophisticated and specialized equipment and also needs a well-trained experimenter to appropriately conduct the test.

Sine Wave Grating

The grating charts are discussed as follow:

1. Arden Plates: This plate was developed by the American Optical Contrast Sensitivity System. It consists of six printed plates, each with a sine wave grating of a different spatial frequency. The plate is raised gradually to uncover the pattern at increasingly higher contrast levels until the observer identifies the pattern. Because of the subjective nature of this test, this method is now considered by most to be less reliable than other methods.

2. Vision Contrast Test System (VCTS): This system was developed by "Vistech Consultants." It displays data in much the same way as CRT or video displays (computer generated gratings). It consists of a variety of grating patches arranged in 9 columns of progressively diminishing contrast levels (marked as 1-9), and 5 rows of different spatial frequency levels $(1.5,3,6,12$, and 18 cycles per degree), or cycles per degree, to 
measure sensitivity at a particular object size (marked as A-E, Figure 7). Each patch contains oriented sine wave (fuzzy grey bars) tilted in the left, right, or vertical directions. At the bottom, there are a series of patches to give the observer examples of possible slanted directions. This Vistech chart is printed by advanced photographic and printing techniques in order to present calibrated levels of contrast. In addition, this system is available in near and far formats. The VCTS is designed so that it can accurately measure contrast sensitivity under normal room luminance of 30-70 fL. (approximately $100-240 \mathrm{~cd} / \mathrm{m}^{2}$ ). When tested, the observer is asked to indicate the lowest contrast patch visible and to describe which way the bars are slanted. Responses for each row are then plotted on a special data form (the VISTECH Contrast Sensitivity Evaluation Form) to allow easy comparison with normal vision results. The normal range of results given by the manufacturer are: in row A from target 5 to target 7 , in B from 5 to 7 , in $\mathrm{C}$ from 4 to 7, in D from 3 to 7, and in E from 1 to 6 (Mantyjarvi et al., 1998). Commercially, the VCTS is available in two formats, near test (V6000) and far test (V6500). Compared with the plate method, this VCTS chart is considered to be more reliable. This particular chart is not suitable for individuals with astigmatism, because the gratings are oriented.

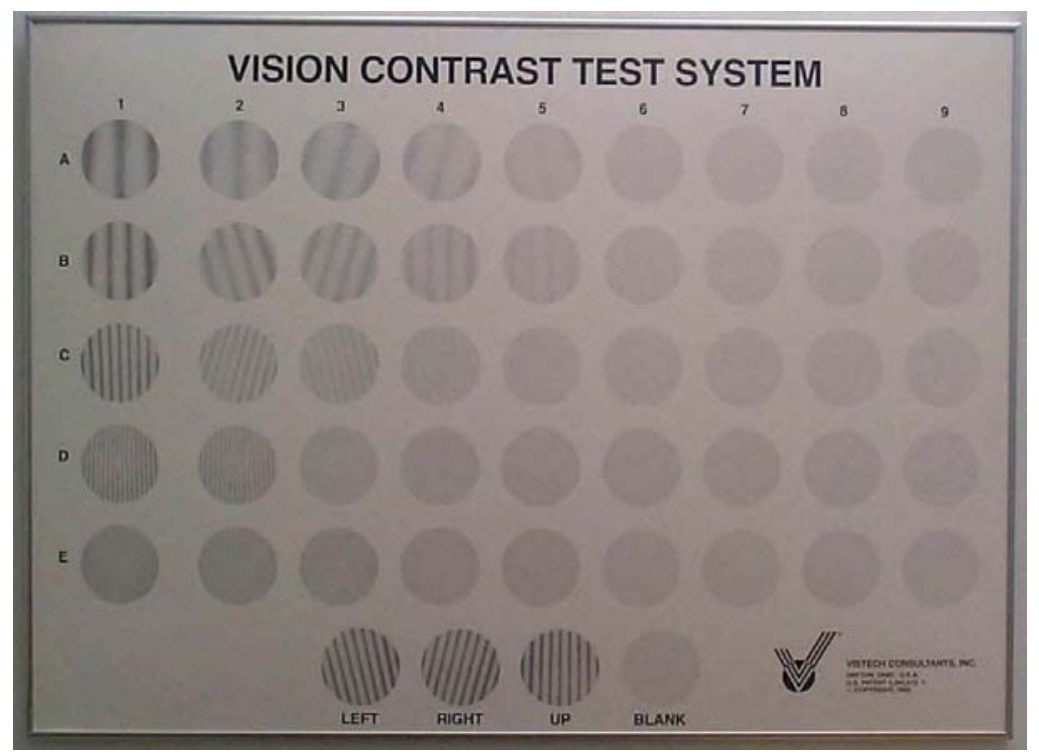

Figure 7: The VCTS Chart. 
3. Functional Acuity Contrast Test (FACT): This test was developed with a slight change from the VCTS by altering the grating patches so that the edges are smoothed into a gray background, which improves the sensitivity and quality of the test. Like VCTS, the chart is also composed of five special frequencies and nine levels of contrast. The observer determines the last grating seen for each row.

4. Vector Vision Testing Methods: The method was developed by "Vector Vision." In this method, a variety of slides are placed over a back lighted screen. The experimenter continually controls the illumination level of the screen and asks the observer to indicate when the gratings are visible.

\section{$\underline{\text { Low Contrast Letter Charts }}$}

Based on the Snellen Visual Acuity Chart, various contrast sensitivity tests are developed including:

1. Regan Low Contrast Letter Acuity Chart: The chart is simply a reduced contrast version of the Snellen chart. There are many charts in various contrast level (e.g., 100\%, $50 \%, 12.5 \%$, and $5.25 \%$ ). Tests are conducted in the same way as a typical Snellen test.

2. Pelli-Robson Chart (Pelli et al., 1988): This chart provides various Sloan letters, including $\mathrm{C}, \mathrm{D}, \mathrm{H}, \mathrm{K}, \mathrm{N}, \mathrm{O}, \mathrm{R}, \mathrm{S}, \mathrm{V}, \mathrm{Z}$, that are arranged in 6 letters per line. All letters are the same (large) size but varied in contrast level (higher contrast on the top fading to the bottom). Note that there are 3 letters presented in the same contrast level to allow more trials ( 3 trials in this case). The chart is very simple to use. It can be administered by any literate person. In testing, the observer is asked to read the letters one by one, starting from the highest contrast level.

The test is stopped when the observer misses two or three letters in a single contrast group and the score is based on the contrast level, defined in Weber's ratio (of the last group in which two or three letters were correctly read). The Pelli test score is replicated in logarithm in scales of participant's contrast sensitivity unit. For example, a score of 2 indicates a contrast sensitivity of 100 percent or the observer was able to read 
at least two of the three letters with a contrast of 1 percent $(1 / 100)$. This chart has been extensively validated. Furthermore, the method is found to be sensitive to visual loss and relatively insensitive to changes in focus, viewing distance, and illumination.

\subsubsection{Contrast Sensitivity vs. Visual Acuity}

Both of these measurements are somewhat different from each other. The purpose of acuity measurement is to estimate the finest detail that an individual can resolve, whereas contrast sensitivity provides information on the individual's ability to detect patterns of all sizes (need not be the finest one). By measuring visual ability at different aspects, it is possible to find a person who has little or no deficit in visual acuity, but demonstrates measurable contrast sensitivity problem.

Contrast sensitivity test can detect some visual impairment aspects that can not be found by acuity test. For example, persons with cataracts, glaucoma, as well as multiple sclerosis, may not be decreased in their visual acuity, but show disability in contrast sensitivity test (Regan, 1991). One distinction between these two visual abilities is that while visual acuity concerns only an ability to see high contrast objects, the contrast sensitivity is concentrated also on ability to see low contrast objects.

\subsubsection{Contrast Sensitivity and Driving Performance}

Many daily tasks are dependent on our ability to see objects against their backgrounds. This is particularly true with the visually guided tasks such as driving and walking. Among these tasks, some hazards occurred are ones with very low contrast situations such as curbs, step-ups and step-downs, stairways, and drop offs. Therefore, good contrast sensitivity is needed to detect and avoid these accidents especially in older adults (Schieber et al., 1992).

In experimental studies, there are fewer studies on contrast sensitivity and driving than on acuity. Nonetheless, the existence of relationship between contrast sensitivity and driving accident has been found (Owsley et al., 2001; Wood et al., 1993; Wood \& Troutbeck, 1992). 


\subsection{Psychophysics Method}

\subsubsection{Three Main Methods}

In order to measure absolute threshold levels for any stimulus, there are three main methods that can be used.

\section{Method of Constant Stimuli}

In this first method, the experimenter will predefine a set of stimulus levels (usually 5-9 different levels). These levels will need to cover the range of the lowest level that is expected to be slightly below the threshold to the highest level that is expected to be slightly above the threshold. These levels will then be presented to the observers randomly and they will report whether they perceive the stimulus or not for each individual presentation.

The collected data then is plotted using the stimulus intensity as $\mathrm{X}$ axis and percent of "yes" or "perceivable" responses on the $\mathrm{Y}$ axis. Normally, results will be found in the S shape curve. This curve is called the "Psychometric Function". This method gives highly accurate estimates of the absolute threshold level, however, is time consuming (Norton, Corliss, \& Bailey, 2002)

\section{Method of Limits}

In this second method, the test will start from some level below the threshold point and continually increase until the observer reports it as perceivable. This trial is called "Ascending Trial". After that, a "Descending Trial" will be performed by starting at the point higher than expected threshold and continually decrease until the observer reports it as unperceivable. Whether to start at the farther or nearer point from the expected threshold shall be randomly selected so that observer could not develop any expectancy of giving a response.

Typically, several trials of ascending and descending trials will be used and the intensity between the points at which responses get reversed will be averaged to get a single value of absolute threshold. Moreover, to ensure the accuracy of the observer's reversed responses, two successive reversed responses are usually required. An example of observer's responses using this method is shown in the Table 1. 
Table 1: Example of Observer's Responses in Method of Limit

\begin{tabular}{|c|c|c|c|c|c|c|}
\hline \multirow{2}{*}{$\begin{array}{c}\text { Stimulus } \\
\text { Level }\end{array}$} & \multicolumn{6}{|c|}{ Trial number } \\
\hline & $1(+)$ & $2(-)$ & $3(+)$ & $4(-)$ & $5(+)$ & Average \\
\hline 1 & No & & No & & No & \\
\hline 2 & No & No & No & & No & \\
\hline 3 & Yes & No & No & & Yes & \\
\hline 4 & No & Yes & Yes & No & Yes & \\
\hline 5 & No & Yes & Yes & No & & \\
\hline 6 & Yes & Yes & & Yes & & \\
\hline 7 & Yes & Yes & & No & & \\
\hline 8 & & Yes & & Yes & & \\
\hline 9 & & Yes & & & & \\
\hline 10 & & Yes & & & & \\
\hline Transition & 5.5 & 3.5 & 3.5 & 5.5 & 2.5 & 4.1 \\
\hline
\end{tabular}

Note: $(+)$ indicates ascending trial, and (-) indicates descending trail

\section{Method of Adjustment}

In this last method, observer is actually the one who adjusts the stimulus intensity level. The experimenter will only set the first level and ask the observer to adjust the intensity level until the stimulus become perceivable or unperceivable depending on whether the starting point is beyond or under expected threshold level. This particular method is used mostly when the stimulus intensity can be varied continuously.

A problem that could occur with this technique is when the intensity controller has some obvious position that may allow observer to anticipate location of the threshold level. To cope with this deficit, using a dial that has no numbers and a variable amount of slip (certain amount of adjusting dial will not yield the same amount of changed intensity). However, one advantage over other two methods is in observer's participation. In this last method, participant will less likely to get bored as could occur in the other methods. Similar to the method of constant stimuli, the level at which observer reports $50 \%$ of the time as detectable will be considered as the absolute threshold.

\subsubsection{Controlling Bias and Guessing Responses}

Bias or guessing responses can frequently occur and affect the measured value adversely. For example, when the observer has a strong bias toward saying either "yes" or "no" for some reasons, there can be a preponderance of guesses in one direction or the 
other. To control these potential problems, catch trials could be introduced as appropriate. Catch trials are trails that obviously above or below the level of threshold intensity to see if the observer presents the expected response.

\subsection{Glare}

\subsubsection{Definition}

Glare is the phenomenon of exposing to the bright light source(s), which considered sufficiently greater than eye's adaptability level, within the observer's visual field of view. Although it may not necessarily impair visual performance, glare could at least cause the sensation of annoyance or disturbance to that person. By recognized definition, when presented glare induces distracted feeling, it is called "Discomfort Glare." However, when the presented glare eventually leads to visual impairment and causes measurable decrease in our visual performance (e.g. loss ability to see for a few seconds, decrease in visual detection performance), it is called "Disability Glare." Normally, disability glare would be accompanied by discomfort glare. Though having the widely recognized definition as stated above, poor understanding of what people mean when they say they have problems with glare still exist (Lennie \& Van Hemel, 2002).

In real life, glare is one of the most problematic factors associated with the driving task. It can happen throughout the day at anytime. At night, a driver may experience various sources of glare, such as the street lighting, the oncoming car's headlights that are directed to the driver's eyes, named as "direct glare" and the following car's headlights, that reflected from rear-view mirrors to the driver's eyes, named as "reflected glare". On the other hand, bright sunlight is one of the glare sources during the daytime. These possible disruptions of vision can impede the detection and reading of navigational signs against a bright sky, and affect the detection of low-contrast curbs, seeing objects on the white pavement. All these situations could eventually lead to higher probability of car accidents. Additionally, glare is found to effect working performance when workers have to work under the condition of glare such as working with computer terminal in the veiling glare situation. 


\subsubsection{Physiology in Glare Exposure}

Physiologically, glare occurs when there are some extents of light scattering in our eyes. These scattered lights are perceived as a veil over the scenes or objects we are intentionally looking at. It is this veil that actually reduces objects visibility by reducing their contrast (Fry, 1955). This phenomenon becomes worse in people with increasing light scattering condition such as aging individuals.

As we age, the eye lens will gradually degenerate and develop inhomogeneities pattern. The cornea clarity as well as vitreous body will also change. These degenerate alterations cause variations in the index of lens refraction and result in more scattering of stray light over the retina (Weale, 1963; Wolf, 1960), and all these physiology changes lead to the enhanced negative glare effect. Some recognized age effects are listed below:

1. To see objects after being exposed to glare, elderly people could require stronger stimuli intensity.

2. Their glare resistance is also diminished (through Wolf Glare Tester by Pulling et al., 1980).

3. Their recovery time after glare exposure is found to be longer (the average at 9 seconds for older group compared to 2 seconds in younger group (Corso, 1981). Unfortunately, this inexorable change is irreversible and the only corrective treatment is by removing the eye lens through special surgery. Researchers have also attempted to investigate some other involuntary physiological changes. Berman (1994) investigated the EMG responses of a few facial muscles and found that the presence of a small bright, uncomfortable glare source produced demonstrable changes in EMG signals.

\subsubsection{Factors Influencing the Level of Glare}

According to some research findings (Bullough, 1999, 2002) several factors are known to affect our glare perception level. Some recognized factors and their influences are listed as follow:

1. Glare source luminance: Increasing the glare source's luminance will increase the effect of glare in both disability and discomfort.

2. Background luminance: Decreasing background luminance will increase the effect of disability glare. 
3. Angle between the observer's line of sight and the direction of the light source: Decreasing this angle will increase the effect of glare since it's directly related to the intensity of the scattered light (Lennie \& Van Hemel, 2002).

4. Glare source size: Glare source size is found to affect only discomfort glare but not disability glare (Alferdinck, 1991; Sivak, Simmons, \& Flannagan, 1990).

5. Color of light source: Comparing between yellow and white headlamps of equal intensity, it was found that yellow headlights are considered less discomfort feeling though less visibility (Bullough, 1999).

6. Difficulty of task: As the task difficulty levels increase, perceived glare feeling increase (worse or more annoyance) than when one performs some other easier task (Sivak et al., 1991).

7. Age: As mentioned in the previous section, older age group tends to be negatively affected when dealing with disability glare (e.g. measuring with contrast sensitivity). However, there is a contradiction regarding discomfort glare feeling. In 1950, Petherbridge \& Hopkinson have concluded that although sensation of discomfort glare varies from participant to participant, it does not appear to be related to age. Later on, Sivak et al. (1991) has concluded differently that the discomfort glare experienced does depend on age.

8. Previous exposure to glare source: People with different past experience would feel and perceive glare level differently (Sivak et al., 1991).

\subsubsection{Measuring method}

\section{Disability Glare}

To measure disability glare, experiments are usually tested based on various visual-related tasks, which require participants to perform the task with the presence of glare source(s). Example of these tasks includes visual acuity test, low-contrast visual acuity test, contrast sensitivity test, tracking task, and detecting task. In 2002, Lennie \& Van Hemel suggested the importance of participants screening to participate in the glare related experiments. One underlying reason is the fact that people who perform poorly under low-contrast conditions will usually perform even more poorly under glare. 
For these glare tests, the glare sources can be either directly pointed or/and reflected to participant's eyes (to simulate both direct and reflected glare conditions). They can be some small, bright glare sources fixed at one location relative to the test task or a more extensive glare source surrounding the task. Regardless of glare source arrangements, participants will be required to perform the tasks while just sitting and exposed to the glare. Examples of dependent variables in this type of experiment are number of targets missed, reaction time, time required to recover to a pre-established level of visual function (Ranney et al., 1996). Also, the participant's contrast sensitivity when exposed to glare source is used as a dependent measure (Bullough, 2002).

Though a number of glare tests have been developed presently, no standardized methods exists (Lennie \& Van Hemel, 2002). Some of the available tests include:

1. Miller-Nadler Glare Test: This test uses a slide projector viewer that provides the surround glare around the Landolt's circle targets of various contrasts. An aperture will allow observer to see the target (Miller \& Nadler, 1990).

2. The Brightness Acuity Tester (BAT) (Holladay et al., 1987): The tester is a hemispherical bowl with controlled glare source held close to the observer's eye.

3. The Berkeley Glare Test (Bailey et al., 1991): The test uses low-contrast acuity charts against a large, bright background. Visual acuity is tested both with and without presence of glare source. Disability glare index is defined by the difference between these two visual acuities.

4. Van den Berg and colleagues (De Waard et al., 1992): They have introduced a test utilizing a flickering light in ring-shape. This flickering light is used as the glare source, while a small spot inside the ring, which flickers in counter phase to the glare source, and is used as the target. The test is conducted adjusting the target until it appears as no flicker.

5. CSV-1000HGT (Halogen Glare Test): This test is introduced by Vector Vision. It is particularly designed for evaluating disability glare. The testing instrument is composed of two halogen glare sources attached to the side of a contrast sensitivity slide. The glare source can be adjusted to simulate many glare conditions (e.g. on-coming halogen car headlights as seen at night from 150 feet). 
Among these, the BAT technique is quite popular in cataract surgery evaluation. Moreover, other disability glare tests that have not been widely discussed or studied in the literature include the InnoMed true vision analyzer (TVA), the VisTech VCT 8000, the EyeCon 5 (Neumann et al., 1988), the Humphrey Automatic Refractor Model 570 (Beckman et al., 1992), and the Opthimus glare test (Martin, 1999).

\section{Disability Glare in Automotive Studies}

As being a major problem in driving situations, disability glare has been studied for long in the automotive field. Many researchers have applied the task performance measurement into the driving simulator (e.g. Ranney et al., 1999; 2000). In their studies, participants are asked to perform various type of tasks as would be executed in real driving tasks (e.g. steering wheel control, compliance speed with legal sign along the road way, detect for humans or objects and avoid sudden accidents, etc.) while they actually drive the simulated car in manipulated scenario and weather conditions. Then, performances of those tasks are measured to indicate disability glare level (e.g. reaction time, number of misses or required distance to detect pedestrian dummies having either high or low contrast against the street background, measure of steering wheel and lane position variability). Note that all of these dependent variables are normally designed to avoid interference with primary driving task.

However, one disadvantage that simulated studies confront is the big difference in driver's risk-taking behaviors as compared to the real-life circumstances. That's why some other researchers have decided to test with real driving on streets or field experiments instead (e.g. Sivak \& Olson, 1982; Theeuwes, 2002).

\section{$\underline{\text { Discomfort Glare }}$}

For discomfort glare, its functional impact is poorly understood and there are no standard and accepted tests existing currently. However, it is often measured through the use of subjective glare rating scales (e.g. visual analog rating scale, and nine-point De Boer Scale) even though some problems are repeatedly found with their use. For example, a recent study has shown that the scale is practically useless for predicting actual driving performance. This particular study demonstrates the fact that rating 
responses are only dependent on the glare illuminance at the observer's eyes (Theeuwes, 2002). Furthermore, high between-subject variability is often found in the use of rating methods (Berman et al., 1994; Theeuwes, 2002). Similarly, tolerance to headlight glare as measured by the subjective glare tolerance was found not to be related either to individual physiological thresholds or participants' ages (Pulling et al., 1980).

These problems have led to a distinct desire to objectively measure discomfort glare level. In 1994, Berman et al. has introduced the use of electromyographic techniques (EMG) to measure observer's facial muscle activities (Orbicularis Oculi and Supercilli) during the exposure to glare situation. His findings demonstrate the increasing in muscle activity as a response to conditions of increased glare level. Using this similar manner, Murray, (2001) from U.K. also applied the EMG technique and developed a new device to measure discomfort glare level.

In this proposed study, the rating method is used to measure discomfort level of observers. The discomfort glare rating scale decided to use is the revision of the De Boer scale, the most widely used scale in the field of automotive and public lighting (De Boer, 1967a). This revision scale is developed by Wierwille, Lee, \& Dehart et al. (2003) by including more description of the scale terms through the addition of sentences explaining those terms in tolerance time interval that observers could make their decision on (e.g. "The level of glare is a little bothersome. I might want to look away in less than a minute"). Also, instead of 9 point scale, the revised rating method is added up to 17 possible responses. This revised scale is shown in the appendix $\mathrm{F}$ at the end of this report. Lastly, the scale is reversed so that the higher the rating means the worse in the glare subjective experience.

\subsubsection{Glare and Driving Performance}

Since our visual contrast sensitivity is decreased due to the existence of glare sources, our detection performance, especially with low-contrast object is found to be low. Therefore, any driving tasks that require the ability to visually detect objects will gain harmful effects to some extent, such as reading of signs, and detection of critical objects (e.g. pedestrian, objects in rear mirror). This fact was demonstrated by required longer reaction time to correctly detect simulated pedestrians along the roadside with 
glare sources in Ranney experiment (2000). Not only the degraded detection task performance, but some other driving tasks were also shown to get effect, such as laneposition variability, reduced speed on curves, and most consistently, increased steering variability (Ranney, 1996). Theeuwes (1996) also found similar results in worsening driving performance.

However, all of the experimental settings referred to above were employed with the disability glare level since it was known to directly effect on our visual capability. With this glare level, estimating for the degree of deterioration can be quantitatively calculated through Vos method (Vos, 1984). Distinctively, Theeuwes (2002) was concentrating on only discomfort level. In his study, lower in detection performance was also found with the increasing of glare. He found that drivers require shorter detection distance in continuous low glare condition, perform with more steering wheel reversals, and gain more number of target missed. As discussed earlier, participant's adaptation strategy to reduce the unpleasant feeling is claimed to be primary reason. For example, as they move their head position away from the glare source, the probability to miss target would be increased. With this finding, it becomes more difficult to differentiate discomfort glare from disability glare by the degree of vision impairment since low glare level still could indirectly cause some impoverishments on visual tasks.

Nonetheless, it's crucial to point out that adaptation strategy is not always employed but rather depends on how difficult is the driving task also. In the study, he found that driving behavior of drivers who are not acquainted with the road environment or when driving on harsh road conditions will be worse. This finding further supports the effect of discomfort glare as dependent on task difficulty levels discussed earlier.

Furthermore, prolonged glare exposure is, too, one of the researcher's concerns dealing with driving performance. The first attempt to study this particular effect is done by Schiflett et al. (1969). Giving participants with a continuous direct glare source while driving on the 3 hour driving simulator, they found that participant's reaction time to an auditory stimulus is increased over the period of time. On the other hand, Ranney (1996), who in testing his experiment with intermittent glare source, found no evidence to support that there are some impairment of target-detection ability as well as driving performance. Note that increasing of subjective sleepiness over time is also shown to be 
another result of this latter experiment (Pulling et al., 1980; Ranney et al., 1999; Schiflett et al., 1969).

\subsection{Trait and Personality}

\subsubsection{What is Personality Trait}

Personality traits are known as the building block of the human personality. It views a person's personality as consisting of some number of different dimensions. Therefore, when people are referred as having a different personality from others, they are different in how strong or weak their personality is in each concerned dimension. As of today, various theories are introduced and though they are all not in agreement on defining these "blocks", all agree on existence of the blocks.

Once personality traits are defined, they mostly consistent in the way individuals behave, feel, and think. Thus, they may be used to summarize, to predict, and to explain a person's responses to the situations. However, it is important to point out that there are some situations that can also result in different behaviors as mentioned by Allport (1937): "traits are often aroused in one situation and not in another." In sum, trait theorists suggest that people have broad predispositions to respond in certain ways and that there is a hierarchical organization to personality.

\subsubsection{Growth and Development Effects on Personality}

Most trait researchers believe that trait stability is quite high throughout adulthood but for childhood, there still be some difference in views (Caspi \& Roberts, 1999; McCrae \& Costa, 1997; Roberts \& Del Vecchio, 2000). Initial studies in the United States suggested small, though significant, age effects. In particular, older adults score significantly lower in neuroticism, extraversion, and openness and higher in agreeableness and conscientiousness than adolescents and young adults in their early twenties (e.g. college age) (Costa \& McCrae, 1994). The decrease in A and C (agreeableness and conscientiousness) also fits with findings from the literature on juvenile delinquency (related to low A and low C and Eysenck's Psychoticism), which decreased markedly after adolescence. However, these findings are ambiguous because 
the observed differences may reflect not age changes but cohort differences, that is, differences due to generation effects associated with growing up during different factors (e.g. growing up during WWII) rather than age factors.

In contrast, some researches have supported the concept of stability in personality traits especially in extraversion scale. Using same group of participants, Schuerger et al., (1989) conducted personality tests at two different time periods. The finding has shown that even over 20 years time interval, the correlation between the first and second tests is still a resounding .64. Nonetheless, in another line of research (e.g. Helson \& Moane, 1987), there is a focus on personality consistency at the transition age from adolescence to early adulthood since it is during this period that issue of identity is crucial for an individual. As expected, the correlation between two different conducted personality tests is ranging only from .32 to .41 , which is substantially lower than the one tested with adult groups.

\subsubsection{Five-Factor Model of Personality}

Nowadays, many researchers agree that traits can be usefully organized in terms of five bipolar dimensions (e.g., Costa \& McCrae, 1992; Goldberg, 1981; John, 1990). These dimensions are widely known as the "Big Five" or the "Five-Factor Model of Personality (FFM)". All five dimensions are organized by using factor analysis method $^{1}$ to see which traits go together and form a single board characteristic. Moreover, all five factors have been shown to possess considerable reliability and validity and to remain relatively stable throughout adulthood (McCrae \& Costa, 1994). These five board dimensions are Neuroticism (N), Extraversion (E), Openness (O), Agreeableness (A), and Conscientiousness $(C)$. The first letter of these dimensions are spell out as OCEAN (John, 1990). As this particular research will pay attention to only two dimensions; Neuroticism and Extraversion, more details on these two scales are discussed here.

\section{$\underline{\text { Extraversion }}$}

While extraverts are normally described as sociable, impulsive, cheerful, carefree, assertive, and adventurous, introverts are known to be more formal, more reserved,

\footnotetext{
${ }^{1}$ Factor analysis is a statistical analysis method used to determine which variables are related but independent from others.
} 
remain distant from others, more sensitive to pain, become fatigued more easily, and tend to be more careful but less fast. Based on these defined characteristics, it is not surprised that Campbell \& Hawley (1982) have found that extraverts often chose to study in library locations (shows more outgoing personality) that provided external stimulation than did introverts. They also took more study breaks than did introverts.

Besides mentioned disagreeable characteristics between these two groups of personality, arousability is of interest for this particular research. It is suggested that introverts are more arousable than extraverts. The underlying reason of this difference is introduced by Eysenck \& Eysenck (1967). According to his theory, arousability difference emanates from deviation of the functioning of the ascending reticular activating system (ARAS) ${ }^{2}$ since it is this system that regulates the balance between excitatory mechanisms (causes an individual to be awaken, aroused, and alert) and inhibitory mechanisms (produces the reverse effect). The function of this system is to regulate the amount of information or stimulation that goes into the brain. A person whose ARAS causes him or her to be chronically over aroused is an introvert and s/he will have more probability to receive stimulations more than they need. Eventually, introverts would end up seeking to avoid these over aroused situations.

An example of research to support this concept is done through the "lemon drop test" (Eysenck \& Eysenck, 1967). They found that with the same amount of lemon drop on participants' tongues, introverts salivate more, equivalently to more arousable. After this lemon drop test, a few more researches have demonstrated similar results (e.g. Gilbert \& Hagen, 1985, on nicotine stimulus level; Wigglesworth \& Smith, 1976, on auditory stimulus level). However, this finding actually limits to only if the stimulus is still in low intensity range. By being easier to arouse, introverts would reach the upper threshold of arousal sooner. Then, any increasing stimulus intensity would result in decreasing in arousal level. Thus, introverts would then become less aroused than extraverts. Note that the point, which arousal turns toward opposite direction is called "transmarginal inhibition" and it becomes a major problem for this theory as to where exactly the transition point would occur.

\footnotetext{
${ }^{2}$ The arousal level of the ARAS determines cognitive reactivity to external stimuli
} 
At the same time, many researches have concentrated on finding the preferred stimulation level and compared the results provided by introverts and extraverts (e.g. Davis, Hockey, \& Taylor, 1969; Weisen, 1965). It has been repeatedly found that not only introverts get aroused faster, but also reach their optimal level sooner (required less amount of stimulus intensity to reach their preferred level). As a result, introverts are normally easier to get over aroused than extraverts, which turn into unpleasant feelings. On the other hand, as extraverts require more energy to reach their desired level, the stimulus that is lower than their optimal point will put them into a bored state.

\section{$\underline{\text { Neuroticism }}$}

This personality dimension is sometimes labeled by both its positive and negative poles. The positive pole, named as "Emotional Stability", includes traits such as calm, relaxed, and stable while at the opposite pole, named as "Neuroticism", includes anxious, depressed, emotional, and apprehensive. A person, who breaks down under stress, easily get bodily aches and produces more widely varying emotional extremes is the one who is on the neurotic end. By having a tendency to feel depress and lack of stability, neurotics can also reflect more complaints about things. Evidently, they do score lower on happiness, well-being health, and other related attributes (McCrae \& Costa, 1991; Watson \& Clark, 1984). The underlying reason for neuroticism differences is also hypothesized to be from biological difference in nervous system functioning.

Interestingly, these two personality dimensions were repeatedly found related to each other. In 1982, Eysenck concluded that the vast majority of neurotic patients who have high neuroticism would be more likely to have low extraversion scores. In other words, someone who seems to be more depressed and unstable would have a tendency to be careful, seek to avoid high stimulation, and so on. This existence of possible relationship between neuroticism and extraversion leads to the question of advertised independent ability among all five personality factors (Funder, 2001). Additionally, it was found that extraversion and neuroticism are strongly and differentially related to positive and negative affect, respectively (e.g. Costa \& McCrae, 1980; Larsen \& Ketelaar, 1991; Watson \& Clark 1992). 


\subsubsection{Personality Assessment via Questionnaire}

Besides the lemon drop test discussed earlier, some more recent methods are measured by the use of personality inventory, or personality questionnaire. A variety of questionnaires have been developed since 1980's to measure personality in the discussed of Five-Factor Model, such as the Goldberg's (1992) bipolar inventory measuring the Big Five with trait adjectives, the NEO-Personality Inventory Revised (NEO-PI-R) developed by Costa \& McCrae (1992), the Personal Characteristics Inventory, the Hogan Personality Inventory, and the Multidimensional Personality Questionnaire.

However, as researchers conducted via the Internet becomes of greater importance, numbers of personality trait questionnaires are available for public used online. One major difference of the online questionnaire versions from the traditional ones is the shorter length of questionnaire that is considered very critical for online testing to avoid boredom feeling of responders and eventually discard the test. Also, as being able to test from any connected Internet line, the amount of time used to conduct this type of questionnaire is considerably less than the paper and pencil method. Among these available online questionnaires, the International Personality Item Pool (IPIP) developed by Goldberg, (1999) is the one that currently comes in to public interest. Besides being available in original English format, the IPIP items have also been translated into nine other languages including Spanish, German, Dutch, Swedish, Indian, Norwegian, Romanian, Hungarian, and Serbian.

This IPIP is available in two versions; an original long version and a new shorter version. This test is developed in parallel to the scores produced by the Big-Five model. The original version contains up to 100 items while the newer one contains 50 items only. Although the short version meets professional standards of reliability (openness has cronbah's alpha $=0.74$, consciousness 0.84 , extraversion 0.88 , agreeableness 0.76 , neuroticism 0.83) (Buchanan, 2001), the original version is found to be more reliable.

Comparing with the NEO-PI-R, a widely used commercial inventory, Mcbride (2001) concluded that the IPIP consists of items less than half of the NEO-PI-R without providing less than half of the information. Her finding also shows that the IPIP scales actually provide a greater amount of information as well as precision per item than does 
the corresponding NEO-PI-R. In addition, the longer version of IPIP was earlier found to gain .63 to .93 correlations with the NEO-PI-R scales (Goldberg, 2000). Clearly with more items, this longer version would require approximately longer time to complete (approximately 40-60 minutes) whereas the shorter one require only about 15-25 minutes.

For the purpose in this research, the IPIP 100 item version is used because of its acceptable reliability and free public use. Nonetheless, since only neuroticism (or "Emotional Stability") and extraversion facets are focused, 40 related items (20 items for each facet) are extracted and put together in one questionnaire in random ordered. Each item, which describes personality descriptions, is designed to get responses in the 5 point Likert scale format, ranging from "Very Inaccurate" to "Very Accurate" as the scale showed substantial decrease in precision and information when response scales were dichotomized away from the original format (Mcbride, 2001). A complete list of the IPIP items used in this study is provided in Appendix C.

For scoring process, each item score will first be adjusted for direction. For example, if the item is "+ve" direction and a person report as "Very Accurate", s/he will gain 5 points. On the other hand, if the item is "-ve" direction and a person report as "Very Accurate", s/he will gain 1 point. Then, each relevant item score will be added to gain the total score of particular personality dimension. Therefore, having a higher score will suggest stronger personality in that dimension.

\subsubsection{Individual Differences and Driving Performance}

Many studies were focused on the individual differences as a factor of driving accidents and received ticket involvement. The list of individual dimensions is as follows. Note that there is controversy in some of the findings:

1. Age: Young people, particularly males, are found to be involved with more accidents (Jonah, 1986). The reason may be the tendency to overestimate their abilities in young group (Kogan \& Wallach, 1964) as well as underestimate dangers and perceive lower risks in potential threatening situations (e.g. Finn \& Bragg, 1986). 
2. Trait-based individual differences: In 1970, Shaw \& Sichel reported accidentprone drivers to be neurotic extraverts (high score in neuroticism and extraversion personality dimensions). However, controversial results still may be reported by finding no significant relation between these personality traits and driving accident (e.g. Cellar et al., 2000; Craske, 1968; Fine, 1963; Shaw \& Sichel, 1970; Singh, 1978; Wilson \& Greensmith, 1983).

3. Impulsivity: This personality facet also found to discriminate between accidentfree and accident-involved drivers (Trimpop \& Kirkcaldy, 1996; Beirness, 1993; Mayer \& Treat, 1977; Pelz \& Schuman, 1968; Schuman et al., 1967). The accident prone group would be the drivers with immediate sensations while the accident free group would be the drivers with anticipating goals.

4. Risk taking: Risk taking is also found to be a good factor to predict drivers' accident involvement (Trimpop \& Kirkcaldy, 1996). The more risk taking the person does, the more accidents s/he will tend to be involved in.

5. Conscientiousness: Arthur \& Graziano found inverse relationship between conscientiousness and driving accident involvement in 1996. That is, relative to those less conscientious, highly conscientious individuals are less likely to be involved in driving accidents.

Besides influencing on an overall driving performance, individual differences also found to relate with some other work performances (e.g. sale and service employees (Piedmont \& Weinstein, 1994), financial service manager (Salgado \& Rumbo, 1994). 


\subsection{METHOD}

\subsection{Participants}

A total of 36 individuals ( 9 low extraversion scorers, 9 high extraversion scorers, 9 low neuroticism scorers, and 9 high neuroticism scorers) were recruited from 78 volunteers (students at Virginia Polytechnic Institute and State University and the general population in Montgomery County, Virginia). The participants' ages ranged from 19 to 75 years at the time of experiment. Participants were recruited by e-mail and/or verbal conversation solicitation as a part of a research project designed to determine a discomfort glare feeling factor. They were informed about monetary compensations. The informed consent was given to participants to inform them about the objectives and procedure of the experiment as required (Appendix G). Several tests were used as the initial screening tools to ensure that all participants fulfilled the following criteria:

1. Participants must have had the extreme score in either the neuroticism or extraversion trait dimensions as measured by the 40 IPIP items. With this criterion, only those who fell within the range of either $1 / 3$ extreme highest or $1 / 3$ lowest personality score were included. Please refer to Appendix A for the screening results.

2. Participants must have had visual acuity at $20 / 50$ or better in the nighttime visual acuity test. With this criterion, only those who had average visual acuity 20/50 or better from 5 different testing distances were selected. 20/50 was set as a criterion since the test was conducted in a simulated nighttime environment where the visual acuity decrement is expected.

3. Participants must have had contrast sensitivity level in a normal range for all of the frequency ranges and for both eyes. The recommended norm for Vistech Chart V6500 was used as the criteria in this screening.

Only participants who fulfilled all three mentioned criteria were included in the contact list for the experiment and were contacted to come for the experiment session. 


\section{$\underline{\text { Sample Size Calculation }}$}

Estimation of required size for these experiments proceeds from estimates of inter-subject variability in glare discomfort rating responses obtained from previous work (Toyota, 2002). Power calculation was performed by focusing on sample sizes large enough to determine differences between high and low neuroticism score participants with high probability. The general test statistic for two populations would be the standard two-sided t test, for which the power of the test (Neter et al., 1996) is given by:

$$
\text { Power }=\mathrm{P}\left\{\left|\mathrm{t}^{*}\right|>\mathrm{t}(1-\alpha / 2 ; \mathrm{n}-2)|\delta|\right\}
$$

Where $\delta$ is the non-centrality parameter or a measure of the distance between the means of rating scores from high and low neuroticism scores:

$$
\delta=|\mathrm{A}-\mathrm{B}| / \sigma \sqrt{ }(2 / \mathrm{n})
$$

Where $\sigma$ is the standard deviation of the distribution of rating scores, and $\mathrm{n}$ is the number of participants in each group.

The difference between $\mathrm{A}$ and $\mathrm{B}$ (or the minimum difference which is important to detect with high probability) was assumed here to be 1.3 (Lockhart, 2002), and the standard deviation in glare rating score was approximately 1.02. Specifying that $\alpha=0.05$, 9 participants in each of the personality groups would be sufficient to detect the specified differences in glare rating responses with risks of Type I error of 0.05 and Type II error of $<0.3$ (Power $>0.7$ ). By requiring 9 participants in each personality group, a total of 36 participants was necessary (i.e., 9 high neuroticism scorers, 9 low neuroticism scorers, 9 high extraversion scorers, and 9 low extraversion scorers). 


\subsection{Apparatus}

The list of equipment used in the experiment is shown below:

1. Adapted IPIP questionnaires (Appendix C: Project Questionnaire)

2. Vistech Chart V6500 version B (for contrast sensitivity test)

3. Black plastic piece

4. 5 Sets of Landolt Circles Test for the distances of 300, 100, 70, 50, $30 \mathrm{~cm}$. Each set has 9 circles that demonstrate 9 different visual acuity levels including 20/20, 20/25, 20/30, 20/35, 20/40, 20/45, 20/70, 20/100, 20/200 (Appendix D: Landolt's C-Circle Chart)

5. Several diffusing filters to control laptop screen luminance

6. Laptop PC (with powerpoint slide on White color and with LABVIEW created 20/200 VA Landolt's C target)

7. Photometer luminance meter (Model: Minolta CS-100)

8. HID headlamp with shade box (large lunch box size)

9. Filters to control headlamp brightness

10. DC Power Supply (12 V. Fix)

11. Tripod (Headlamp Stand)

12. Goniometer

13. Laser Pointer

14. Head Chin-rest

15. Adjustable Height Chair

16. Fixed Height Table

17. Illuminometer

18. A clock with nap time function

19. Adapted De Boer Subjective Glare Rating (Appendix F: Discomfort Glare Rating Scale)

20. Black plastic at the size of 4 in. $x 4$ in. 


\subsection{Experimental Settings}

\section{$\underline{\text { Personality Test }}$}

The participants were either seated in the normal light condition where possible distractions (e.g., noisy sound) were kept at minimum or seated on their own comfort at their computer terminal. The first setting was used with the participants who came to university for another experiment and were interested in participating in this study, while the second setting was used with the new group of participants. The first group filled out the questionnaire using paper and pencil. Note that in the actual questionnaire, the questionnaire's title was written as "Project Questionnaire" rather than "Personality Questionnaire.”

\section{$\underline{\text { Visual Contrast Sensitivity Test }}$}

The visual contrast sensitivity test was conducted under normal room luminance, $100-240 \mathrm{~cd} / \mathrm{m}^{2}$ as measured by a photometer luminance meter at all four chart corners. The benefit of ensuring the light level was to effectively compare results with the available norm. Participants were positioned at $10 \pm 1$ feet away in front of the Vistech chart throughout this test. A piece of black plastic was hold by the participant to cover one eye as the test was conducted using only one eye at a time.

\section{$\underline{\text { Visual Acuity Test }}$}

The visual acuity test for screening purposes was performed in the same environment as the glare test discussed next (ambient light of 3-5 lux). This test was repeatedly performed with a total of 5 different distances as shown in Figure 8. Five sets of acuity tests were changed according to five participant positions (Appendix D: Landolt's C-Circle Chart). Several filters were applied in front of the laptop screen to control target luminance at $4 \mathrm{~cd} / \mathrm{m}^{2}$ for all five testing distances. 


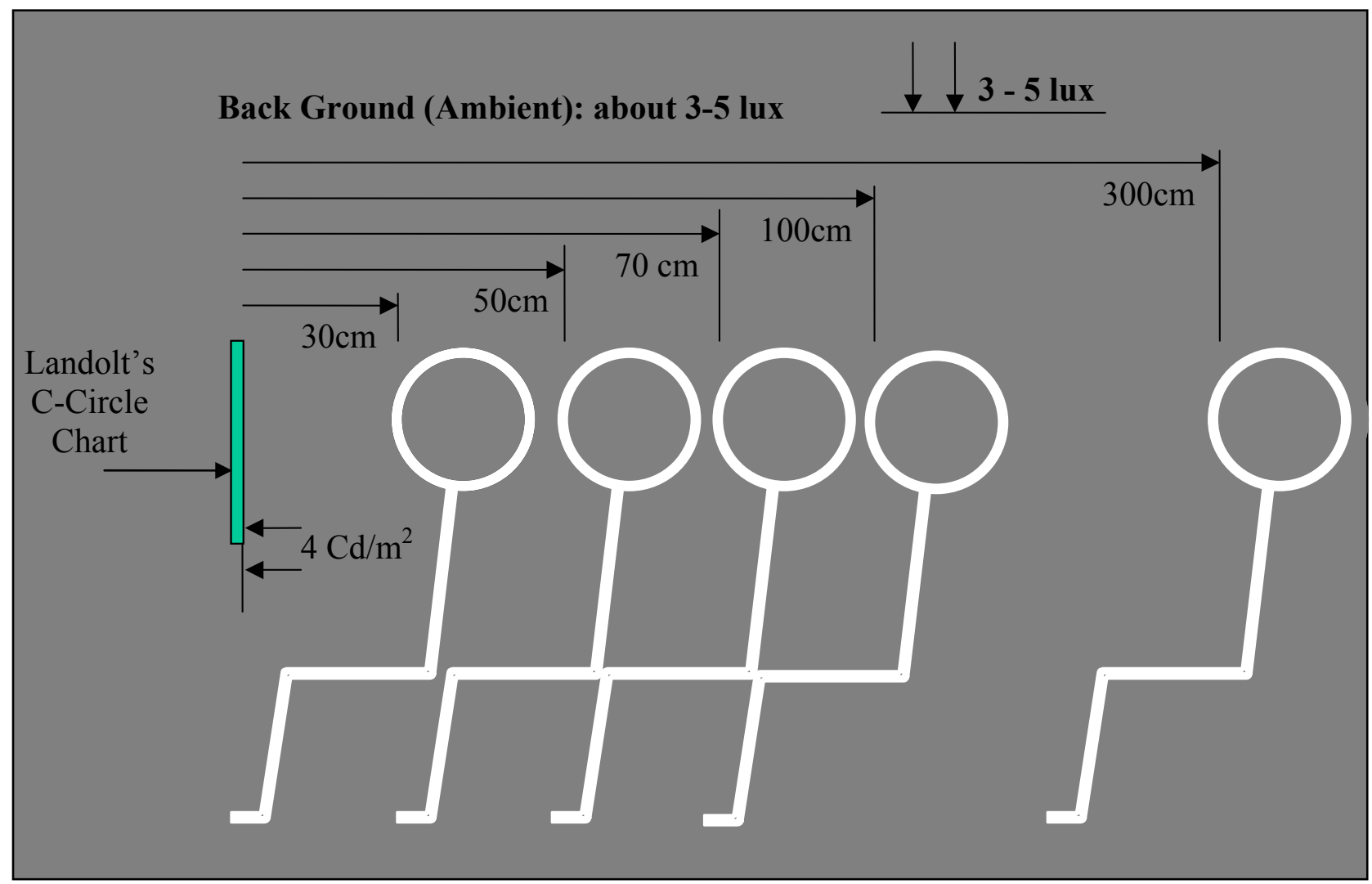

Figure 8: Experimental Settings for Visual Acuity Test.

\section{$\underline{\text { Glare Test }}$}

The simulated nighttime room was used in this experiment. The light sources for ambient illumination were evenly spaced to provide uniform illumination to the work area and ensure that it fell within the range of $3-5$ lux at all times (an illuminometer was used to measure the lighting from the plane tangent to the workbench at the participant's eye height). The room had flat black painted walls to minimize reflected glare. The floors were also blackened with black felt carpet.

The height of headlamp aperture and the middle of Landolt's $C$ target were set to the same level at all times. Note that the aperture of the headlamp was controlled at 0.75 $\mathrm{cm}$ and located 10 degrees away from the participant's line of sight in the left direction. This setting was designed to simulate the actual situation when an oncoming car is located at $50 \mathrm{~m}$ away. 
A laptop presented with a Landolt's C target was placed in line with the headlamp enclosure for all conditions. The participant's eye level was adjusted to be in line with the line of HID headlamp ray. The illumination level on the target located at this position was measured to ensure the least veiling glare from the headlamp.

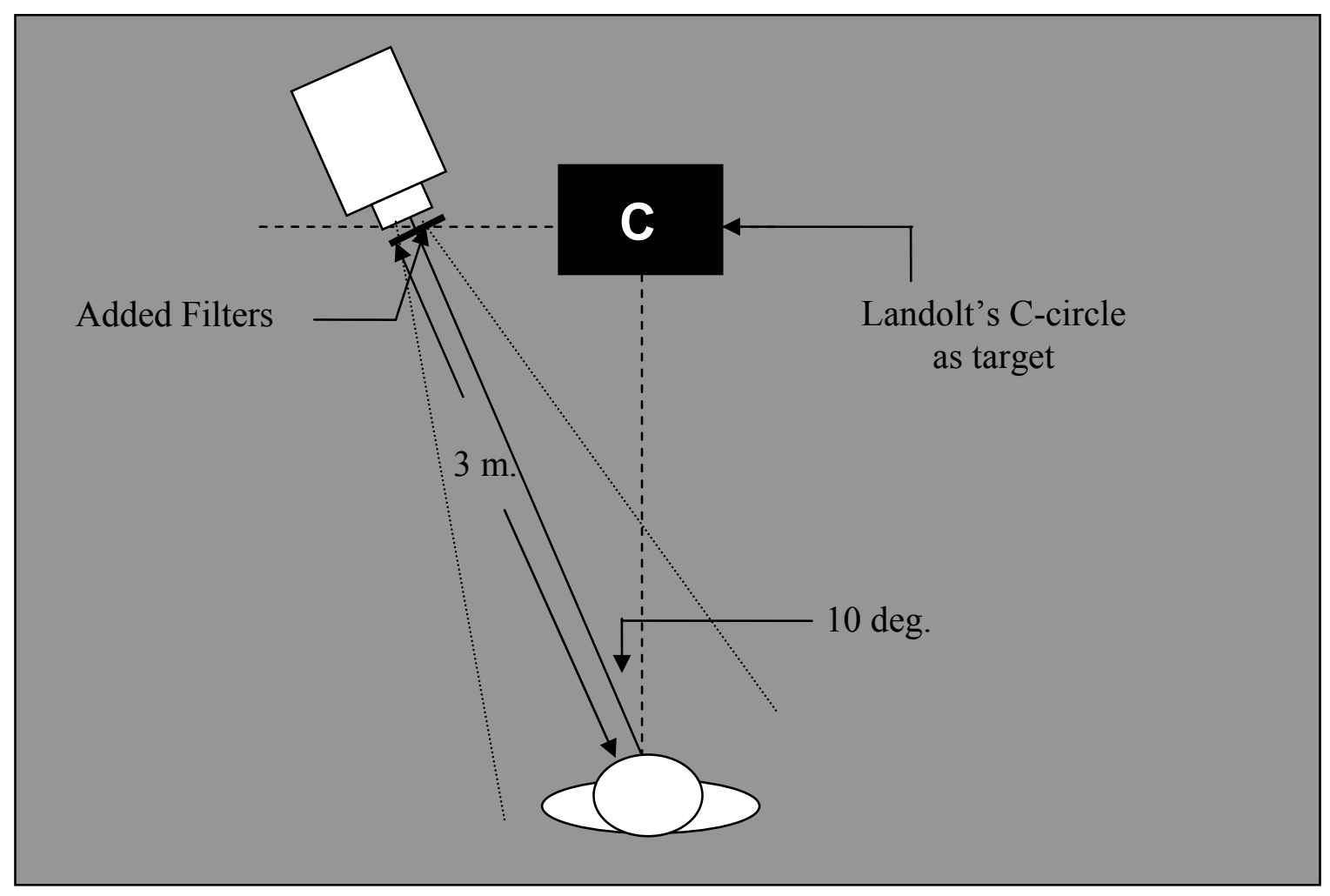

Figure 9: Experimental Settings for Glare Test (top view).

The size of the target was designed using LABVIEW program based on the size of visual acuity test for $20 / 200$ or 0.1 , which is the cut off point for legal blindness. The participant's position was set using the chinrest to ensure consistency among all participants.

\subsection{Procedure}

\subsubsection{Screening Session}

As mentioned, all participants were firstly screened to ensure at an extreme personality (in either neuroticism or extraversion) and adequate visual function in terms 
of visual acuity and contrast sensitivity. The screening session was conducted using the following procedure:

\section{Personality Test}

Volunteers were asked to fill up the personality questionnaire. Two major methods were used.

1. For the participants from the other study: In normal light condition, participants were given with the adapted IPIP questionnaire (Appendix C). Instruction was given in the top of given questionnaire. Any further questions and explanations were told according to the questions participants may have. At all times, there was no intervention from experimenter except for answering to participant's questions.

2. For the new group of participants: The questionnaire was sent via e-mail as the attachment through various mailing lists (e.g., university dormitory, Blacksburg senior). Volunteers who interested in participation the study were asked to fill up the questionnaire and sent back directly to the experimenter.

Then, the scoring of volunteers' answers were done by the excel program utilizing the same method suggested by Goldberg (2000). All scores were sorted and the 1/3 cut off technique was used to identify the extreme score groups (both high and low). Then, the extreme score participants were ranked according to their individual scores. The ranking were given two times according to individual neuroticism and extraversion scores. For example, if one has the lowest score on neuroticism, s/he will receive the rank of -1 on extraversion trait. On the other hand, if one has the highest score on neuroticism, $\mathrm{s} /$ he will receive the rank of +1 on neuroticism trait. The similar ranking method was performed for successive ordering scores. However, when participants had the same score, they all were given with the same ranking.

Next, the ranking order between extraversion and neuroticism trait were compared within one individual to determine his/her trait group. The higher ranking of that individual was the determination for his/her trait group. For example, if a participant 
has the rank of -2 in neuroticism trait and 7 in extraversion trait, this participant will be grouped as low neuroticism scorer. However, if one participant has the equal rank such as -5 in neuroticism trait and 5 in extraversion trait, this participant will be remarked as either low neuroticism or high extraversion scorer. The possible participants who have extreme trait score were contacted to come for the visual screening tests discussed next.

\section{Visual Acuity Test}

Firstly, each participant was allowed with fifteen-minute dark adaptation starting from entering the simulated nighttime room. During this time, the experimenter conversed with the participant, asked questions and tried to make the participant feel comfortable. The experimenter also loaded the white slide (PowerPoint) and put diffusing filters on laptop in front of the screen (Note: Filters are used in order to ensure the luminance level of C-circle at $4 \mathrm{~cd} / \mathrm{m}^{2}$ ).

Then, participant was seated at the $300 \mathrm{~cm}$. away from and directly in front of the PC screen. Participant's chair was adjusted to level participant's sitting eye height with the middle of the laptop screen. Participant was asked to report the smallest Landolt's circle that $\mathrm{s} /$ he can still detect direction of the broken gap (Appendix D). The experimenter wrote the visual acuity corresponded to participant's answer. In case the answer was incorrect, the experimenter asked participant to report the next larger Ccircle). Nine possible results are $0.1,0.2,0.3,0.4,0.5,0.6,0.7,0.8$, and 1.0. After finishing the first distance, procedures were repeated in the same manner for the distance of $100 \mathrm{~cm}, 70 \mathrm{~cm}, 50 \mathrm{~cm}$, and $30 \mathrm{~cm}$ away from PC screen. The set of Landolt's C-circles were changed accordingly to the viewing distance as well (Appendix D).

\section{Contrast Sensitivity Test}

The Vistech contrast sensitivity chart was hung on the wall vertically to the floor. Using luminance meter, the chart was ensured to have a luminance level in the range of $100-240 \mathrm{~cd} / \mathrm{m}^{2}$ for all of its four corners. Once the experimenter finish setting the light, the participant was located at a 10 feet distance $( \pm 1$ foot $)$ away from the chart. Four example patches were introduced to participants to indicate four possible answers in the test (up, left, right, and blank). Then, a black plastic sheet was given to participant to 
cover one of their eyes. Note that the test started from using with the left eye and then the right eye.

For each eye used, participant was asked to determine the slanted direction of bars for all patches starting from A-1 to E-9. For each row, participant was encouraged to guess beyond the last visible patch. The instruction was extracted from Vistech Chart Manual (Appendix E). Record was made on the data form by checking the numbered circle in each column which corresponds to the highest numbered patch the observer see correctly in each row of the chart.

\subsubsection{Actual Experimental Session}

At least one week after the completion of the personality questionnaire, participants were scheduled to come back for the glare session. The one week period was introduced to control any bias feedback that the participant could have on providing subjective glare rating. This session was conducted in the same room with visual acuity test, the ambient light level of 3-5 lux for entire room. For this session, the procedure was divided into two parts as follows:

\section{Familiarize Session}

Each participant experienced an adaptation period upon entering the darkened laboratory for 15 minutes before participating in the experiment. During this period, experimenter gave the explanation about the subjective rating scales by providing descriptive form for all scale anchors. Participant was asked to ensure the understanding of rating scale. A note of rating description was given to participants so that they could refer to it anytime they want during the experiment process (Appendix F). Having this scale, participant was told to report the rating response in associated number ranging from 1 to 9 and the report of half score, such as 7.5, was accepted.

Then, participant was asked to sit on an adjustable height chair. Participants' chin was set on a chin-rest. The height of chin-rest was fixed so that participant's eyes will always be in line with the line of HID headlamp ray. The other settings were as shown in Figure 9. Next, the testing of contrast sensitivity under no glare condition was conducted. For this condition, the headlamp was either turned off or covered with a 
black piece of plastic. The Landolt's C targets were randomly loaded on the laptop screen. The experimenter asked participant to fixate at the middle of target screen throughout the experiment. The experimenter measured the baseline illuminance level at participant's eyes.

After than, participant was asked to perform the contrast threshold test using method of limit as follows. Examples of the instruction were shown in Appendix E:

1. Ascending Trial: Started from the lowest contrast and continually increased the target's contrast level. For every increasing level, participant was asked to report whether they can detect the target or not. If yes, different $\mathrm{C}$ targets, which were different in opening direction, but were the same in contrast level were presented. Participant was asked to report the direction of the opening. Two successive reports of correct answer were the termination of the ascending trial.

2. Descending Trial: Started from the above threshold found in prior step, the experimenter continually decreased the contrast level. For every decreasing level, participant was asked to report whether s/he can detect the target or not. If no, the experimenter increased contrast level up for a step. Then, different $\mathrm{C}$ targets, which were different in opening direction but were the same in contrast level, were presented. Participant was asked to report the direction of the opening. Two successive reports of correct answer were the termination of the descending trial. In this case, the reported reading dial was one step decreasing level.

Note: Total of three trials for each ascending and descending methods were conducted. Contrast sensitivity data received during familiarization session were reported as contrast sensitivity at baseline or no glare condition.

\section{Glare Test Session}

Once finished the familiarization session and answered all related questions that participants might have, the actual glare test began. Participant was seated in the same set up as familiarization session. Then, the experimenter turned on the headlamp or removed the black plastic. The first glare level was manipulated according to predefined counterbalancing plan using a set of filters. Having exposed to the manipulated glare, participant was asked to verbally give the subjective rating utilizing the adapted 
discomfort glare scale provided earlier. Instruction for rating response was shown in Appendix E. After received participant's response, the black plastic was used to cover the headlamp and at least 1 minute resting period was provided. During these times, participant was allowed to close their eyes as well as moving around (e.g., stay out of the chinrest). A nap time clock was used to ensure adequacy of resting duration.

At the end of one-minute period, participant was asked to resume fixating on the monitor and set his or her chin on the chin-rest. The experimenter removed the black plastic and put a set of appropriated filters. Experimenter then ensured the glare setting by measuring the illuminant level at the middle of participant's eyes. Adjusting chin-rest height was performed to ensure that the measured illuminant level was fallen closely to the approximate level. Then, contrast threshold tests were conducted for in the same manner as discussed in familiarization session. Both ascending and descending tests were performed three times. However, a one-minute break was provided between each set of trials and the illuminometer was used to ensure illuminant level at participant's eyes prior to all ascending trials.

The average value of all three trials was reported. Note that all data were reported in dial's number, which were later translated into the contrast sensitivity utilizing method defined in the 3.5.2. Next, the second and third manipulated glare levels were tested following the same steps.

\subsubsection{Pilot Testing}

Before the actual experiment, pilot testing was conducted with two volunteers. No screening test was conducted for these two volunteers. This test was conducted one week before planned trials to have adequate time for any redesign or corrections. The main purposes of the pilot test were to investigate the degree of understanding experimental instructions as well as procedures and to estimate the time duration for each participant. Pilot testing data was also analyzed to ensure the effectiveness of data collection protocol. Note that same procedures followed in the pilot tests as in the experimental session. 


\subsection{Experimental Variables}

\subsubsection{Independent Variables}

$\underline{\text { Glare level }}$

Three levels of glare were used. Simulated glare levels were performed by adding different filters in front of the HID headlamp enclosure as follows.

1 Dim Level: Manipulated by using both $50 \%$ and $30 \%$ filters. The approximate illuminance was 10 lux at the participant's eyes.

2 Moderate Level: Manipulated by using only the $30 \%$ filter. The approximate illuminance was 40 lux at the participant's eyes.

3 Bright Level: Manipulated by using no filters. The approximate illuminance was 110 lux at the participant's eyes.

Personality group

The personality group was defined according to personality scores each individual receive from the IPIP self-assessment questionnaire responses. The criteria used in categorize trait group is the $1 / 3$ method. Each participant was categorized into only one of the four possible trait groups; high extraversion, low extraversion, high neuroticism, and low neuroticism.

\subsubsection{Dependent Variables}

\section{$\underline{\text { Subjective glare rating }}$}

The rating was given by participants utilizing the modified 9-point De Boer scale. The possible responses range from 1 to 9 in 0.5 increments (Appendix F by Wierwille, Lee, \& Dehart, 2003). A lower rating response means lower discomfort perception $(1=$ Not Noticeable, 2 = Just Noticeable, $3=$ Satisfactory, $4=$ Not Quite Satisfactory, $5=$ Just Acceptable, $6=$ Bordering on Disturbing, $7=$ Disturbing, $8=$ Nearly Unbearable, and $9=$ Unbearable).

\section{Contrast sensitivity index (CSI)}

Using the photometer, the luminance of target and its background were measured (in $\mathrm{cd} / \mathrm{m}^{2}$ ) separately for each dial value from 1-100 (Figure 10). 


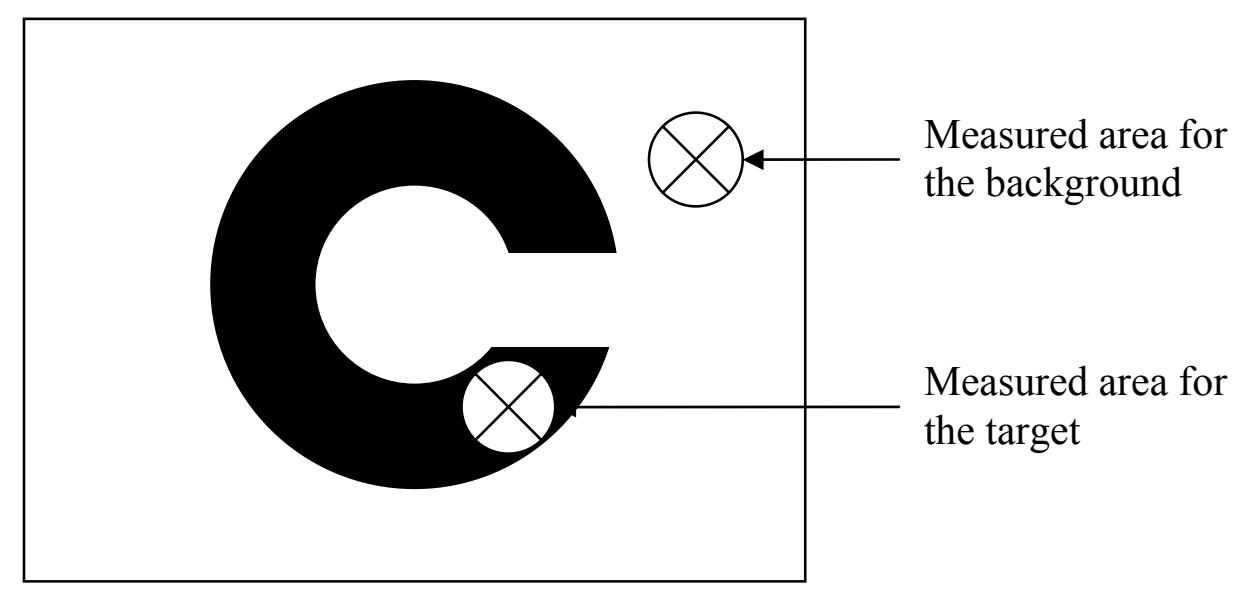

\section{Figure 10: Measured Area for Calculated Contrast Ratio}

Then, the measured values were used to calculate the contrast ratio at the particular dial value using Weber's contrast expression as follow:

$$
\begin{aligned}
\text { Contrast Ratio } & =(\mathrm{L} \max -\mathrm{L} \min ) / \mathrm{L} \max \\
\text { Where: } \quad \mathrm{L} \max & =\text { Luminance of the } \mathrm{C} \text { target } \\
\mathrm{L} \min & =\text { Luminance of the background }
\end{aligned}
$$

Having paired data of dial value and contrast ratio, the polynomial third degree trend line is calculated for the third degree equation relationship. The equation for this relationship is defined as: Contrast Ratio $=-(3 \mathrm{E}-07 \times$ Dial Value 3$)-(7 \mathrm{E}-05 \times$ Dial Value $\left.^{\wedge} 2\right)+(0.0188 \times$ Dial Value $)+0.004$. Note that $R^{2}$ of this equation is 0.9945 (Figure 11).

Then, this equation was used to translate the average dial value reported during the experiment into the related average contrast ratio. The contrast sensitivity was further calculated by the reciprocal of this contrast ratio. For example, in the bright glare level, if an individual reported detecting the target at the average dial value of 24 , his/her contrast ratio would be approximately 0.410733 (using the third degree equation) and his/her contrast sensitivity would be 2.434673 (reciprocal of 0.410733 ). 


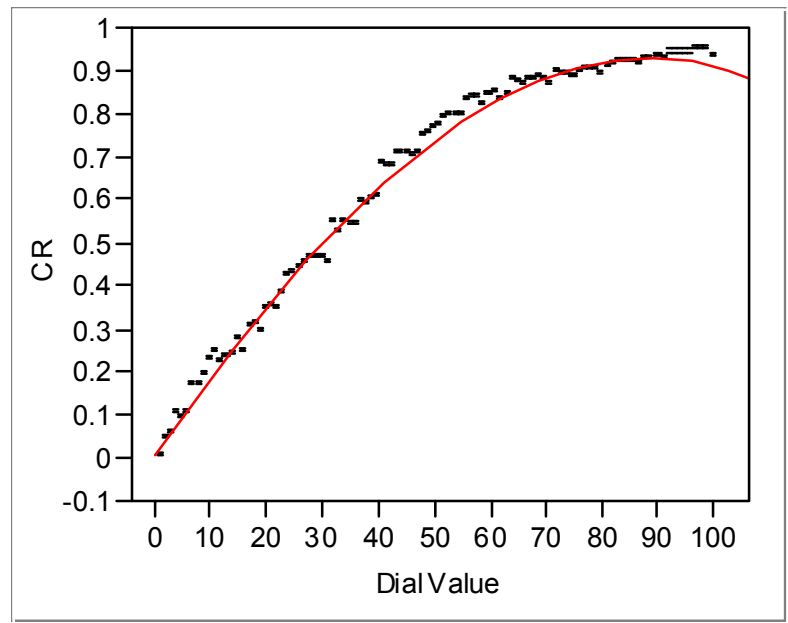

Figure 11: Polynomial Third Degree Relationship between Dial Value and Calculated Contrast Ratio

Finally, the contrast sensitivity index (CSI) was calculated by subtracting the individual's contrast sensitivity of the no glare condition from the one in the simulated glare condition. For this particular example, if an individual in the no glare condition reported detecting the target at an average dial value of 7.5 , then contrast sensitivity for this no glare condition would be 7.095419. Therefore, his/her CSI for the bright glare condition would be 4.6675 (calculated from 7.095419-2.434673). Similar calculations were performed for all glare levels for all participants' collected data.

\section{$\underline{\text { Illumination Level }}$}

The illumination levels were measured at the middle of the participant's eyes using the illuminometer.

\subsection{Design of Experiment}

This study was a 3 x 2 mixed factor design. The within-subject factor was glare intensity level. Total of 3 glare levels were manipulated. The between-subjects factors were personality groups. A total of 4 groups were taken into account as two separate studies: one on the neuroticism trait and the other on the extraversion trait. Therefore, the study could be viewed as two separate $3 \times 2$ mixed factor designs as shown in Table 2 . 
Table 2: Experimental Design.

\begin{tabular}{|l|l|l|}
\hline \multicolumn{1}{|c|}{ Factor } & \multicolumn{1}{c|}{ Factor Type } & \multicolumn{1}{c|}{ Levels } \\
\hline 3 Glare intensity levels & Within-Subject & Dim, Moderate, Bright \\
\hline 2 Neuroticism groups & Between-Subject & High, Low \\
\hline
\end{tabular}

\begin{tabular}{|l|l|l|}
\hline \multicolumn{1}{|c|}{ Factor } & \multicolumn{1}{|c|}{ Factor Type } & \multicolumn{1}{c|}{ Levels } \\
\hline 3 Glare intensity levels & Within-Subject & Dim, Moderate, Bright \\
\hline 2 Extraversion groups & Between-Subject & High, Low \\
\hline
\end{tabular}

In addition, all participants were exposed to the same three glare simulated levels. A set of fixed balanced methods for these three glare levels were used to control any carry-over effect that might occur due to adaptation to the glare situation. The counterbalanced order is shown in the Table 3. The thirty-six participants' numbers are used to refer to all 36 participants. The numbers 1 to 9 indicate the low extraversion group. The numbers 10 to 18 indicate the high extraversion group. The numbers 19 to 27 indicate the low neuroticism group. The numbers 28 to 36 indicate the high neuroticism group.

Table 3: Counterbalancing among Three Glare Simulated Levels

\begin{tabular}{|c|c|c|c|}
\hline \multirow{2}{*}{$\begin{array}{c}\text { Participant's } \\
\text { Number }\end{array}$} & \multicolumn{3}{|c|}{ Glare level ordered } \\
\hline & First & Second & Third \\
\hline $1,7,10,16$ & Dim & Moderate & Bright \\
\hline $2, \quad 11$ & Dim & Bright & Moderate \\
\hline $3,8,12,17$ & Moderate & Dim & Bright \\
\hline $4, \quad 13$ & Moderate & Bright & Dim \\
\hline $5,9,14,18$ & Bright & Dim & Moderate \\
\hline $6, \quad 15$ & Bright & Moderate & Dim \\
\hline
\end{tabular}

\begin{tabular}{|l|l|l|l|}
\hline \multicolumn{2}{|c|}{$\begin{array}{c}\text { Participant's } \\
\text { Number }\end{array}$} & \multicolumn{3}{c|}{ First } & \multicolumn{1}{c|}{ Second } & \multicolumn{1}{c|}{ Third } \\
\cline { 2 - 4 } & \multicolumn{2}{|c|}{ Sumel ordered } \\
\hline $19, \quad 28$ & Dim & Moderate & Bright \\
\hline $20,25,29,34$ & Dim & Bright & Moderate \\
\hline $21, \quad 30$ & Moderate & Dim & Bright \\
\hline $22,26,31,35$ & Moderate & Bright & Dim \\
\hline $23, \quad 32$ & Bright & Dim & Moderate \\
\hline $24,27,33,36$ & Bright & Moderate & Dim \\
\hline
\end{tabular}

In conclusion, two data matrix were used in this study as shown in Table 4: 
Table 4: Data Matrix

\begin{tabular}{|c|c|c|c|}
\cline { 3 - 4 } \multicolumn{2}{c|}{} & \multicolumn{2}{c|}{ Extraversion Score } \\
\cline { 3 - 4 } \multicolumn{1}{c|}{} & High & Low \\
\hline \multirow{3}{*}{ Glare Level } & Rating $_{1-9}$ & Rating $_{10-18}$ \\
& & CS $_{1-9}$ & CS $_{10-18}$ \\
\cline { 2 - 4 } & Moderate & Rating $_{1-9}$ & Rating $_{10-18}$ \\
& & CS $_{1-9}$ & CS $_{10-18}$ \\
\cline { 2 - 4 } & \multirow{2}{*}{ Bright } & Rating $_{1-9}$ & Rating $_{10-18}$ \\
& & CS $_{1-9}$ & CS $_{10-18}$ \\
\hline
\end{tabular}

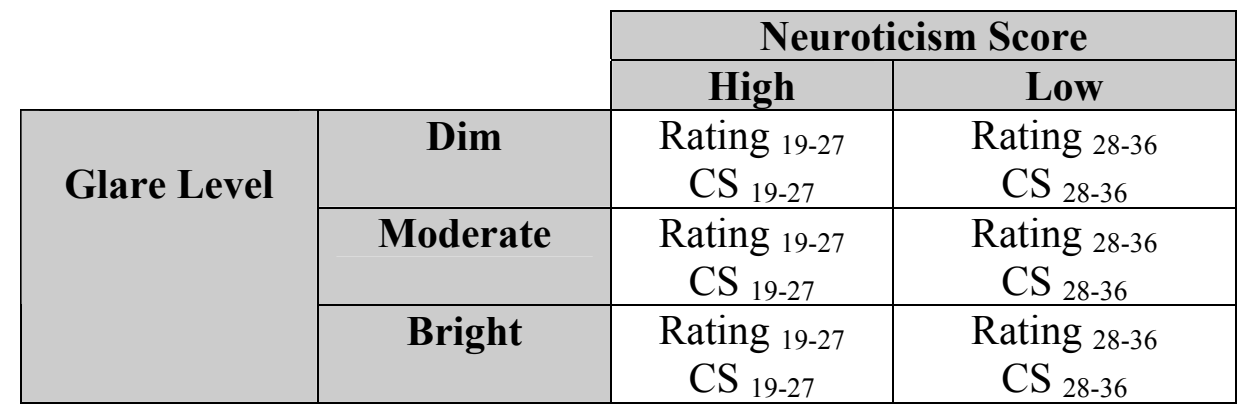

\subsection{Data Analyses}

Data analyses were composed of both multivariate and univariate statistics methods. The multivariate method used was the MANOVA test, while the univariate methods used both parametric and non-parametric statistics, depending on the characteristics of the dependent data and the type of experimental design (i.e., within- or between-subjects). Non-parametric methods were used specifically for ordinal data (rating responses), whereas the parametric methods, mainly ANOVA, were used to analyze both ordinal (rating) and nominal data (contrast sensitivity index and illumination level). Lastly, bivariate analysis was also used to investigate the relationship between two dependent measures.

\subsubsection{Multivariate Test:}

The multivariate analysis of variance test statistics (MANOVA) was used to compare the four personality groups (high neuroticism, low neuroticism, high extraversion, and low extraversion) and three glare levels (dim, moderate, and bright) on dependent measures, the CSI and the subjective rating, simultaneously. 


\subsubsection{Univariate Test:}

The following univariate tests were used to analyze data from the neuroticism and extraversion groups separately, however, in the same manner.

\section{$\underline{\text { Parametric Statistics }}$}

A 3 x 2 (Glare level x Personality group) two-way mixed factor analysis of variance (ANOVA) was performed on each dependent variable with $\alpha \leq 0.5$. These variables include contrast sensitivity index, subjective rating, and measured illuminance. Note that though the illumination level was not used to test any hypotheses, they were analyzed to confirm the appropriate control of the experiment, particularly between the personality groups (e.g., high and low neuroticism groups should expose to the same amount of glare intensity). Additionally, the analysis of the ordinal data (the subjective rating) using parametric methods was for comparison purposes only (whether or not the parametric and non-parametric methods yield different results). Moreover, the post-hoc means separation test (Tukey-Kramer) was performed on all significant effects found with the ANOVA tests. This test was used to investigate Hypotheses 1, 2, and 3.

\section{Non-Parametric Statistics}

Two major non-parametric approaches were used, including the Friedman and the Kolmogorov-Smirnov tests. The Friedman test was used to analyze Hypothesis 4 (As the intensity of glare source increases, the subjective glare experience will also increase) since this hypothesis is a one-way within-subject experimental design (with three levels) and has an ordinal dependent measure. On the other hand, the Kolmogorov-Smirnov test was used two times on the neuroticism and extraversion data to analyze Hypothesis 5 (Participants with high neuroticism scores will report higher subjective glare experience compared to people with low neuroticism scores) and Hypothesis 6 (Participants with high extraversion scores will report lower subjective glare experiences compared to people with low extraversion scores), since both hypotheses are one-way betweensubjects experimental design (with two levels) and have ordinal dependent measures. 


\subsubsection{Bivariate Test}

The bivariate regression analysis was used to investigate the relationship between several pairs of dependent measures as follows:

1. Between the subjective glare rating and the contrast sensitivity index: This first pair was used to investigate Hypothesis 7 (Subjective glare experience will have no significant relationship with the measured contrast sensitivity index).

2. Between the individuals' neuroticism scores and the subjective rating: This second pair was used to investigate Hypothesis 5 (Participants with high neuroticism scores will report higher subjective glare experiences compared to people with low neuroticism scores).

3. Between the individuals' extraversion scores and the subjective rating: This second pair was used to investigate Hypothesis 6 (Participants with high extraversion scores will report lower subjective glare experiences compared to people with low extraversion scores). 


\subsection{RESULTS AND DISCUSSION}

This section starts with the experimental settings evaluation followed by the MANOVA testing results. Then, the univariate and bivariate testing results are arranged according to the seven hypotheses. For each hypothesis, the related results are showed first and are followed by discussion of that particular hypothesis. The seven hypotheses are shown below:

1 As the intensity of glare source increases, the contrast sensitivity index (CSI) will also increase (the visual contrast sensitivity will decrease).

2 Participants with different neuroticism scores will have no significant difference in contrast sensitivity index (CSI).

3 Participants with different extraversion scores will have no significant difference in contrast sensitivity index (CSI).

4 As the intensity of glare source increases, the subjective glare experience (as measured by modified De Boer scale) will also increase.

5 Participants with high neuroticism scores will report higher subjective glare experience (as measured by modified De Boer scale) compared to people with low neuroticism scores.

6 Participants with high extraversion scores will report lower subjective glare experiences (as measured by modified De Boer scale) compared to people with low extraversion scores.

7 Subjective glare experience (as measured by modified De Boer scale) will have low relationship with the measured contrast sensitivity index.

Note that the other results which are not directly related to defined hypotheses are discussed in the Appendices including Appendix A: Screening test results and Appendix B: Secondary results and discussion. 


\subsection{Experimental Setting}

ANOVA was used to analyze the measured illumination level at participant's eyes. One of the major objectives of these analyses is to ensure that the compared high and low trait score groups were exposed to the same (not significantly different) light levels. Results indicated that the manipulated settings were appropriate.

Table 5: Descriptive ANOVA Summary Table for Experimental Setting

\begin{tabular}{llll}
\hline Variable & High Scorer & Low Scorer & P-value \\
\hline Illuminance (lux) - N Data & 60.0163 & 57.4094 & 0.3969 \\
& $(51.7529)$ & $(49.6264)$ & \\
Illuminance (lux) - E Data & 65.2802 & 64.6081 & 0.7075 \\
& $(57.5808)$ & $(55.5432)$ & \\
\hline
\end{tabular}

\begin{tabular}{lllll}
\hline Variable & Dim & Mod & Bright & P-value \\
\hline * Illuminance (lux) - N Data & 9.864 & 41.246 & 125.028 & $<.0001$ \\
& $(1.3475)$ & $(7.8797)$ & $(17.2775)$ & \\
* Illuminance (lux) - E Data & 11.123 & 43.956 & 140.204 & $<.0001$ \\
& $(1.2316)$ & $(6.5119)$ & $(14.675)$ & \\
\hline
\end{tabular}

Illumination between High and Los Scorers

The result indicated no statistically significant differences between the two neuroticism score groups $\left(F_{1,48}=0.7308, p=0.3969\right)$. Similarly, there are no significant differences in measured illumination between two extraversion score groups $\left(F_{1,48}=\right.$ $0.1425, p=0.7075)$.

\section{Glare Level Main Effect:}

The result indicated statistically significant differences in measured illumination among three glare intensities. This conclusion was supported by both neuroticism data $\left(F_{2,48}=35.7164, p=<.0001\right)$ and extraversion data $\left(F_{2,48}=904.8547, p=<.0001\right)$. 


\subsection{MANOVA Test}

\section{Personality Type Main Effect:}

The result indicated statistically significant differences $(p=0.0016)$ between the four personality groups on some of the dependent variables (Table 6, Figure 12).

Table 6: Personality Type Main Effect Test on CSI and Rating

\begin{tabular}{lllrrl}
\hline Test & Value & Exact F & Num DF & Den DF & Prob $>$ F \\
\hline F Test & 0.1717209 & 5.4951 & 3 & 96 & 0.0016 \\
\hline
\end{tabular}

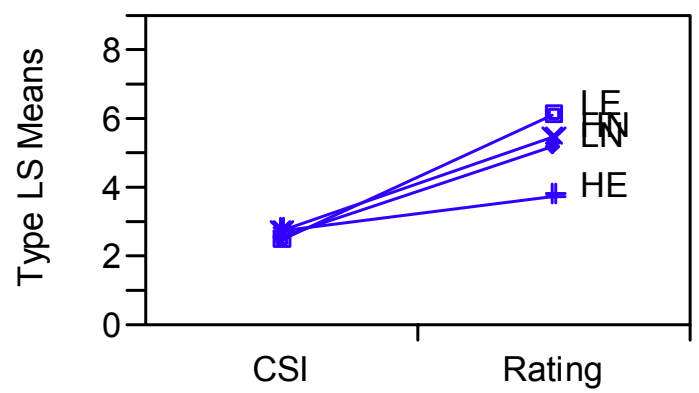

Responses

Figure 12: Personality Type Main Effect on CSI and Rating

According to the means plot above, the difference was expected to be mainly on the rating dependent measure only. Therefore, the hypotheses 2 and 3 were expected to get support. A large higher rating response given by the low extraversion (LE) than the high extraversion (HE) further supported the hypothesis 6. However, the similar rating response between high neuroticism (HN) and low neuroticism (LN) seemed not to support the hypothesis 5 although the $\mathrm{HN}$ did give higher rating responses.

\section{Glare Level Main Effect:}

The result indicated statistically significant differences $(p<.0001)$ between three glare levels on some of the dependent variables (Table 7, Figure 13). The differences were expected to be on both dependent measures. 
Table 7: Glare Level Main Effect Test on CSI and Rating

\begin{tabular}{llllll}
\hline Test & Value & Exact F & Num DF & Den DF & Prob $>$ F \\
\hline F Test & 1.3388966 & 64.2670 & 2 & 96 & $<.0001$ \\
\hline
\end{tabular}

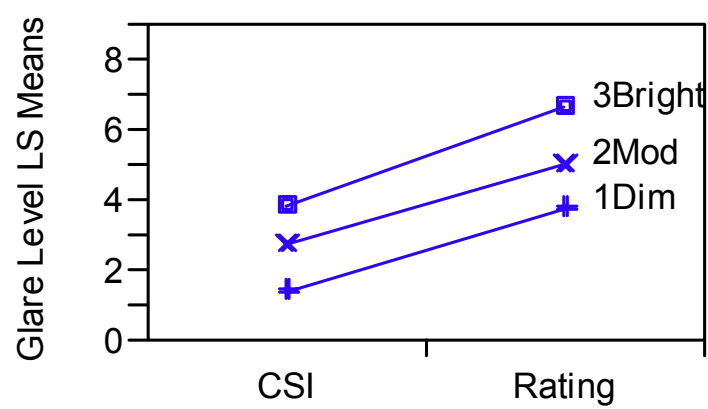

Responses

Figure 13: Personality Type Main Effect on CSI and Rating

Having large difference of CSI and rating responses among all glare levels as shown in the Figure 13, the hypothesis 1 and 4 were expected get support. Note that the higher glare intensities seemed to relate to higher CSI and rating response as hypothesized.

Interaction (Personality Type x Glare Level):

The result indicated no statistically significant personality type by glare level interaction effects $(p=0.9105)$. 


\subsection{Univariate and Bivariate Tests}

The following sections demonstrated the ANOVA and bivariate regression results that related to the seven hypotheses defined in this study. Furthermore, as there are no hypotheses on interaction effect, the interaction analyses are not included here. Note that, for all ANOVA analyses, no interaction effects were found with the current results.

\subsubsection{Hypothesis 1}

As the intensity of glare source increases, the contrast sensitivity index (CSI) will also increase (the visual contrast sensitivity will decrease).

Table 8: Descriptive ANOVA Summary Table for Hypothesis 1

\begin{tabular}{lllll}
\hline Variable & Dim & Mod & Bright & P-value \\
\hline * CSI - Neuroticism Data & 1.35559 & 2.80385 & 3.90213 & $<.0001$ \\
& $(0.816782)$ & $(0.895567)$ & $(0.974473)$ & \\
$*$ CSI - Extraversion Data & 1.37406 & 2.75551 & 3.83849 & $<.0001$ \\
& $(0.99531)$ & $(1.02841)$ & $(1.11262)$ & \\
$*$ CS - Neuroticism Data & 5.03245 & 3.58419 & 2.48590 & $<.0001$ \\
& $(1.12360)$ & $(0.97644)$ & $(0.56271)$ & \\
$*$ CS - Extraversion Data & 4.92036 & 3.54799 & 2.46500 & $<.0001$ \\
& $(1.15042)$ & $(0.90338)$ & $(0.63207)$ & \\
\hline
\end{tabular}

Table 9: Bivariate Regression Analysis Summary Table for Hypothesis 1

\begin{tabular}{lll}
\hline Variable & $\mathrm{R}^{2}$ & P-value \\
\hline * Illumination and CSI - Neuroticism Data & 0.50196 & $<.0001$ \\
$*$ Illumination and CSI - Extraversion Data & 0.46131 & $<.0001$ \\
$*$ Illumination and CS - Neuroticism Data & 0.48358 & $<.0001$ \\
$*$ Illumination and CS - Extraversion Data & 0.47768 & $<.0001$ \\
\hline
\end{tabular}




\subsubsection{Glare Level Main Effect on Contrast Sensitivity Index (CSI) - N Data}

The ANOVA test on neuroticism data indicated statistically significant differences $\left(F_{2,48}=35.7164, \mathrm{p}<.0001\right)$ among glare levels. In general the higher glare levels resulted in the higher CSI (Table 10, Figure 14). Note that the Tukey HSD test indicated statistically differences among all glare levels.

Table 10: Descriptive Summary of CSI on Glare Level Main Effect - N Data

\begin{tabular}{lll}
\hline Glare Level & CSI & Std. Dev. \\
\hline Dim & 1.35559 & 0.816782 \\
Mod & 2.80385 & 0.895567 \\
Bright & 3.90213 & 0.974473 \\
\hline
\end{tabular}

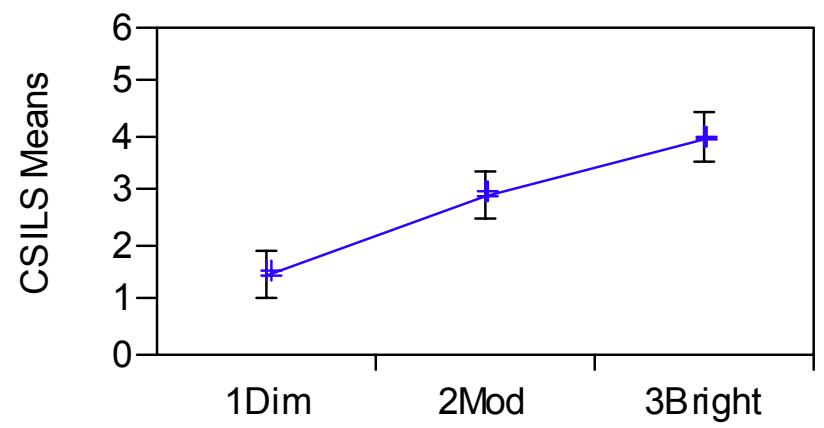

Glare Level

\section{Figure 14: Glare Level Main Effect on CSI - N Data}

Furthermore, the bivariate regression analysis between measured illumination level and individual's CSI was tested. The regression model of this fit, $C S I=1.5506668$ $+(0.0193573 x$ Illumination $)$, indicated statistically different from the simple means model $\left(F_{1,52}=52.4096, \mathrm{p}<.0001\right)($ Table 11$)$. Note that $\mathrm{R}^{2}=0.50196$ indicated that the fit model can explain 50.2 percent of variance. 
Table 11: Analysis of Variance for Significant Linear Relationship between Measured Illumination and CSI - N Data

\begin{tabular}{|c|c|c|c|c|c|}
\hline Source & $\mathrm{DF}$ & Sum of Squares & Mean Square & F Ratio & Prob $>F$ \\
\hline Model & 1 & 50.121148 & 50.1211 & 52.4096 & $<.0001$ \\
\hline Error & 52 & 49.729462 & 0.9563 & & \\
\hline C. Total & 53 & 99.850610 & & & \\
\hline
\end{tabular}

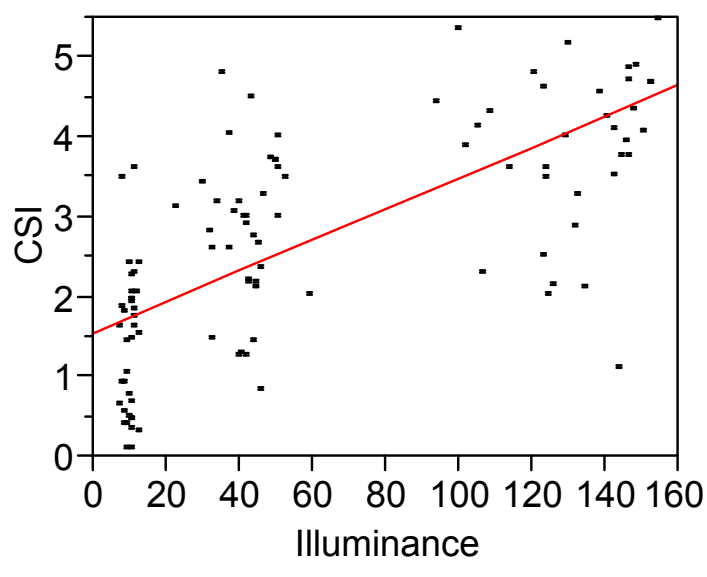

Linear Fit

Figure 15: Scatter Plot between Measured Illuminance and CSI - N Data

\subsubsection{Glare Level Main Effect on Contrast Sensitivity Index (CSI) - E Data}

Similarly, ANOVA test on extraversion data indicated statistically significant differences $\left(F_{2,48}=24.1493, p=<.0001\right)$ among glare levels. In general, the higher glare levels required higher contrast to detect the target (Table 12, Figure 16).

Furthermore, the Tukey HSD test indicated statistically differences among all glare levels.

Table 12: Descriptive Summary of CSI on Glare Level Main Effect - E Data

\begin{tabular}{lrr}
\hline Glare Level & CSI & Std. Dev. \\
\hline Dim & 1.37406 & 0.99531 \\
Mod & 2.75551 & 1.02841 \\
Bright & 3.83849 & 1.11262 \\
\hline
\end{tabular}




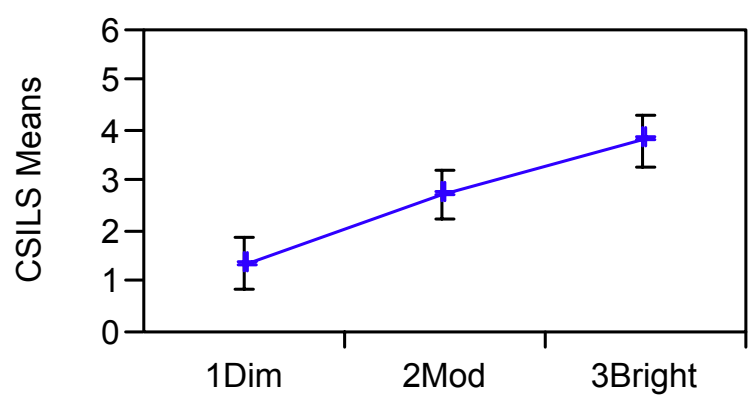

Glare Level

\section{Figure 16: Glare Level Main Effect on CSI - E Data}

The bivariate regression analysis between measured illumination level and CSI given by individual's performance was analyzed. The regression model of this fit, $C S I=$ $1.5216483+(0.017473 x$ Illumination $)$, indicated statistically differences from the simple means model $\left(F_{1,52}=44.1304, \mathrm{p}<.0001\right)($ Table 13$)$. Note that $\mathrm{R}^{2}=0.46131$ indicated that the fit model can explain 46.13 percent of variance.

Table 13: Analysis of Variance for Significant Linear Relationship between Measured Illumination and CSI - E Data

\begin{tabular}{|c|c|c|c|c|c|}
\hline Source & DF & Sum of Squares & Mean Square & F Ratio & Prob $>$ F \\
\hline Model & 1 & 50.81202 & 50.8120 & 44.1304 & $<.0001$ \\
\hline Error & 52 & 59.87311 & 1.1514 & & \\
\hline C. Total & 53 & 110.68513 & & & \\
\hline
\end{tabular}

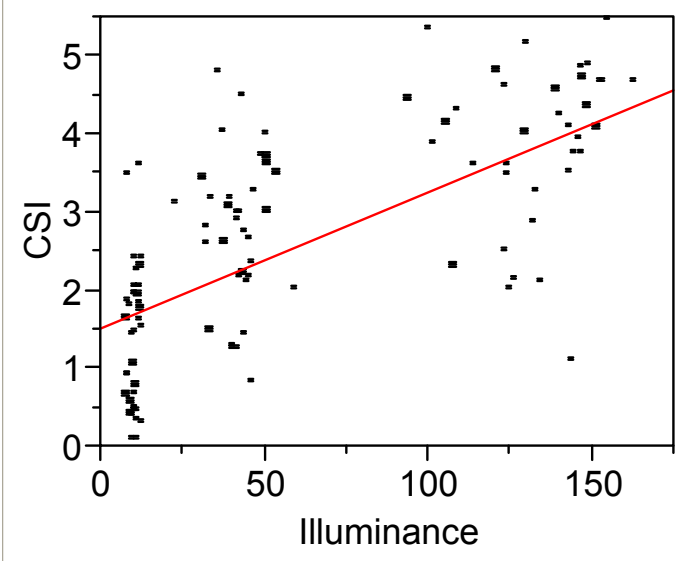
Linear Fit

Figure 17: Scatter Plot between Neuroticism Score and CSI - E Data 


\subsubsection{Glare Level Main Effect on Contrast Sensitivity (CS) - N Data}

The result indicated statistically significant differences $\left(F_{2,48}=35.7432, \mathrm{p}<\right.$ .0001) among glare levels. In general the higher glare levels resulted in the lower CS (Table 14, Figure 18). Furthermore, the Tukey HSD test indicated statistically differences among all glare levels.

Table 14: Descriptive Summary of CS on Glare Level Main Effect - N Data

\begin{tabular}{lrr}
\hline Level & CS & Std. Dev. \\
\hline Dim & 5.03245 & 1.12360 \\
Mod & 3.58419 & 0.97644 \\
Bright & 2.48590 & 0.56271 \\
\hline
\end{tabular}

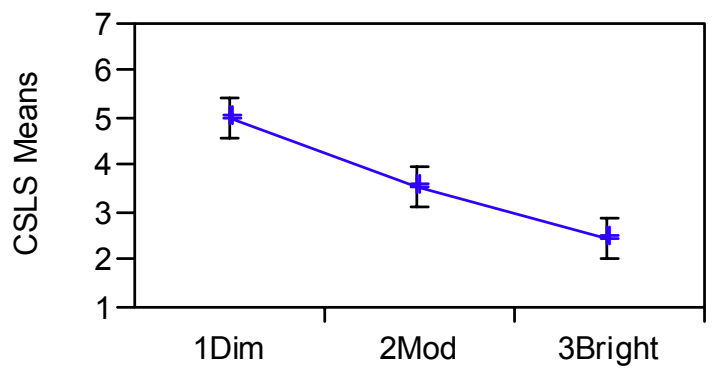

Glare Level

\section{Figure 18: Glare Level Main Effect on CS}

The bivariate regression analysis between two dependent measures for the neuroticism data was performed. The regression model of this fit, $C S=4.8271141$ (0.0191826x Illumination), indicated statistically significant linear relationship $\left(F_{1,52}=\right.$ 48.6924, $\mathrm{p}<0.0001$ ) (Table 15). Note that $\mathrm{R}^{2}=0.48358$ indicated that the fit model can explain 48.36 percent of variance.

Table 15: Analysis of Variance for Significant Linear Relationship between Rating and CS - N Data

\begin{tabular}{lrrrrr}
\hline Source & DF & Sum of Squares & Mean Square & F Ratio & Prob > F \\
\hline Model & 1 & 49.22076 & 49.2208 & 48.6924 & $<.0001$ \\
Error & 52 & 52.56423 & 1.0109 & & \\
C. Total & 53 & 101.78498 & & & \\
\hline
\end{tabular}




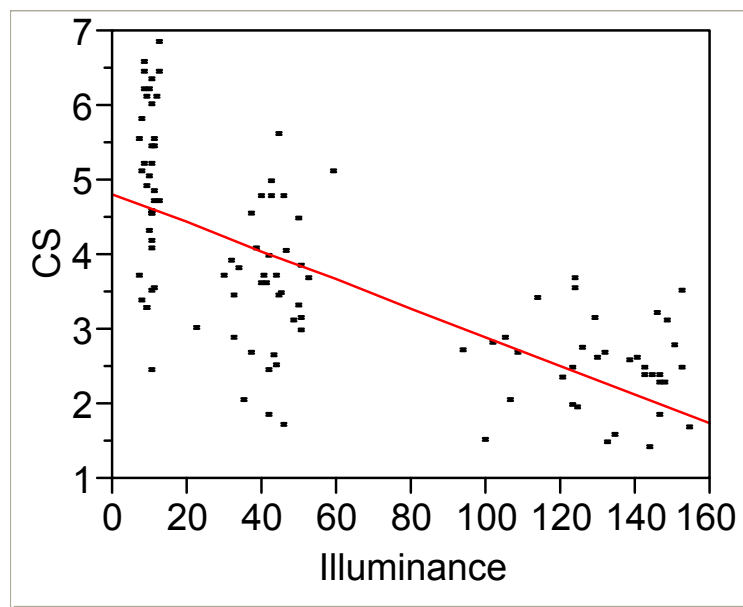

Linear Fit

Figure 19: Scatter Plot between Illumination and CS - N Data

\subsubsection{Glare Level Main Effect on Contrast Sensitivity (CS) - E Data}

The result indicated statistically significant differences $\left(F_{2,48}=30.9583, \mathrm{p}<.0001\right)$ among glare levels. In general, the higher glare levels required higher contrast to detect the target (Table 16, Figure 20). Note that the Tukey HSD test indicated statistically differences among all glare levels.

Table 16: Descriptive Summary of CS on Glare Level Main Effect - E Data

\begin{tabular}{lrr}
\hline Glare Level & Mean & Std. Dev. \\
\hline Dim & 4.92036 & 1.15042 \\
Mod & 3.54799 & 0.90338 \\
Bright & 2.46500 & 0.63207 \\
\hline
\end{tabular}

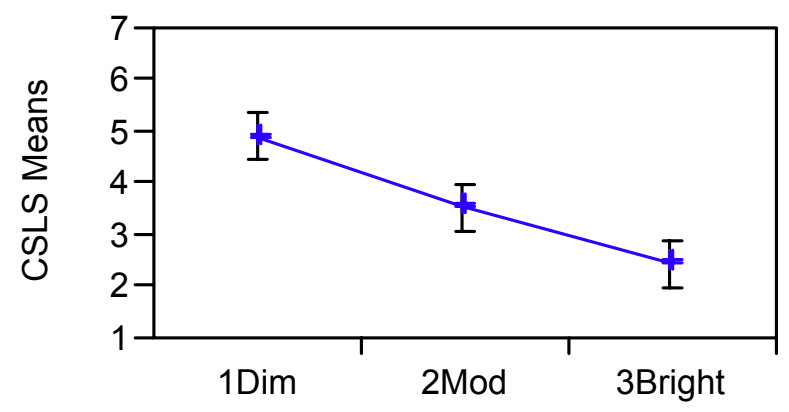

Glare Level

Figure 20: Glare Level Main Effect on CS - E Data 
The bivariate regression analysis between two dependent measures for the extraversion data was performed. The regression model of this fit, $C S=4.7343565$ (0.0167435 x Illumination), indicated statistically significant linear relationship $\left(F_{1,52}=\right.$ 47.5562, $\mathrm{p}<.0001)$ (Table 17). Note that $\mathrm{R}^{2}=0.47768$ indicated that the fit model can explain 47.77 percent of variance.

Table 17: Analysis of Variance for Significant Linear Relationship between

\section{Illumination and CS - E Data}

\begin{tabular}{lrrrrr}
\hline Source & DF & Sum of Squares & Mean Square & F Ratio & Prob $>$ F \\
\hline Model & 1 & 46.657422 & 46.6574 & 47.5562 & $<.0001$ \\
Error & 52 & 51.017228 & 0.9811 & & \\
C. Total & 53 & 97.674650 & & & \\
\hline
\end{tabular}

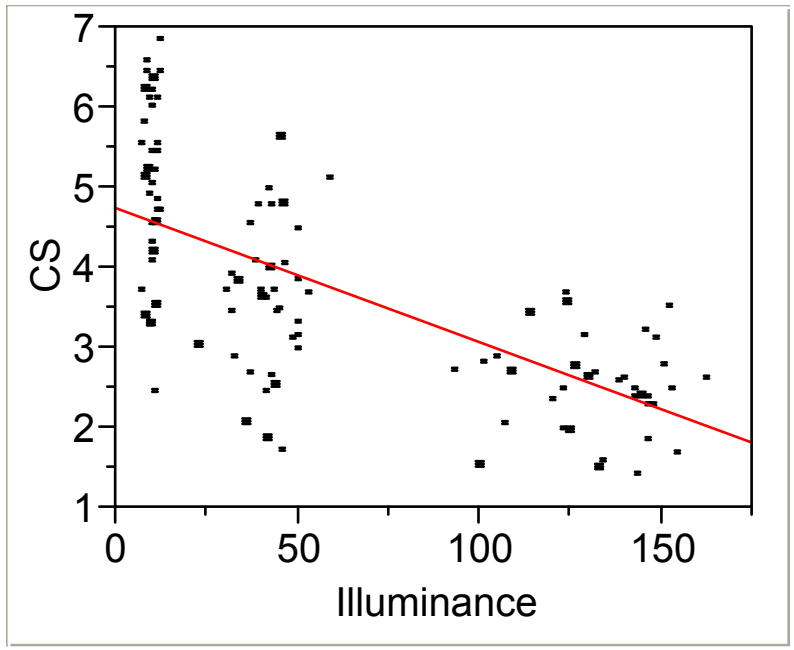

Linear Fit

Figure 21: Scatter Plot between Illumination and CS - E Data

\subsubsection{Hypothesis 1 - Discussions}

Bullough (1999) indicated that the relationship between the observers' CS was related to glare source intensity levels. He found that higher glare levels resulted in lower CS as compared to lower glare levels. Similarly, results from the current research study 
indicated that both CS and CSI were related to the intensity of the glare source: as the intensity of the glare source increased, the CSI increased and the CS decreased.

The above relationships can be explained in terms of physiological changes.

Previous studies (e.g., Miller and Benedek, 1973) indicated that when excessive light is directly pointed to the observers' eyes, the light will pass through various refractive optical components before reaching the fovea region of the retina. As a result, the light become scattered at the fovea. This scattered light will cause blurred images and further lead to inability of the retina-brain combination to discriminate the image in the visual field from its background. Therefore, the requirement for higher contrast between the object and its background is imperative to maintain visual clarity (observer's CS is lower and observer's CSI is higher).

Note that the scattered light at the fovea is found to be dependent upon several factors such as intensity of the glare source, area of the glare source, and its angular proximity to the line of sight (Lennie \& Van Hemel, 2002). In this study, the last two factors were controlled while the first factor was varied to manipulate the glare level independent factor. Thus, the change in CSI is expected to directly relate to the intensity of the glare source only.

\subsubsection{Hypothesis 2}

Participants with different neuroticism scores will have no significant difference in contrast sensitivity index (CSI).

Table 18: Descriptive ANOVA Summary Table for Hypothesis 2

\begin{tabular}{llll}
\hline Variable & High N & Low N & P-value \\
\hline CSI - Neuroticism Data & 2.75557 & 2.61880 & 0.5820 \\
& $(1.40791)$ & $(1.35959)$ & \\
CS - Neuroticism Data & 3.90345 & 3.49825 & 0.1070 \\
& $(1.39589)$ & $(1.37151)$ & \\
\hline
\end{tabular}




\subsubsection{Neuroticism Main Effect on Contrast Sensitivity Index (CSI)}

The result indicated no statistically significant differences $\left(F_{1,48}=0.3072, p=\right.$ 0.5820 ) between the two neuroticism score groups.

\subsubsection{Neuroticism Main Effect on Contrast Sensitivity (CS)}

The result indicated no statistically significant differences $\left(F_{1,48}=2.6979, p=\right.$ 0.1070 ) between the two neuroticism score groups.

In conclusion, Hypothesis 2 was supported by both CS and (i.e., insignificant differences of CSI and CS between high and low neuroticism scorers).

\subsubsection{Hypothesis 2 - Discussions}

Lennie \& Van Hemel (2002) suggested that the amount of adverse effect from glare exposure is dependent on visual performance under the no-glare condition. In this study, vision screening tests under no-glare settings were performed to ensure that participants from both high and low score groups were not different in their screening performances (i.e., visual acuity of 0.4 or higher under nighttime condition, and the normal range of Vistech Contrast Sensitivity Test under the suggested settings). Thus, both the high and low neuroticism score groups' performances under similar glare situations were expected not to be significantly different.

Furthermore, the current study found similar relationship between performance of the screening tests and one under the glare conditions (i.e., poorer performances in the screening tests were related to poorer performances in the glare conditions). The relationships were particularly strong between the CS of the screening test and the glare condition. Please refer to the limitation section for further discussion on the relationship between glare intensity and an individual's neuroticism score. 


\subsubsection{Hypothesis 3}

Participants with different extraversion scores will have no significant difference in contrast sensitivity index (CSI).

Table 19: Descriptive ANOVA Summary Table for Hypothesis 3

\begin{tabular}{llll}
\hline Variable & High N & Low N & P-value \\
\hline CSI - Extraversion Data & 2.79458 & 2.51746 & 0.3455 \\
& $(1.40147)$ & $(1.50234)$ & \\
CS - Extraversion Data & 3.75664 & 3.53226 & 0.3840 \\
& $(1.23034)$ & $(1.48891)$ & \\
\hline
\end{tabular}

\subsubsection{Extraversion Main Effect on Contrast Sensitivity Index (CSI)}

The result indicated no statistically significant differences $\left(F_{1,48}=0.9116, p=\right.$ $0.3455)$ between two extraversion score groups.

\subsubsection{Extraversion Main Effect on Contrast Sensitivity (CS)}

The result indicated no statistically significant differences $\left(F_{1,48}=0.7720, p=\right.$ 0.3840 ) between two extraversion score groups.

In conclusion, Hypothesis 3 was supported by both CS and CSI (i.e., insignificant differences of CSI and CS between high and low extraversion scorers).

\subsubsection{Hypothesis 3 - Discussions}

Similar to the previous hypothesis (Hypothesis 2), the recruitment of participants based on visual performance in standard vision tests is expected to be the reason for these insignificant results (i.e., no significant difference under no-glare condition leads to no significant differences under glare conditions). Please refer to the limitation section for further discussion on the relationship between glare intensity and an individual's extraversion score 


\subsubsection{Hypothesis 4}

As the intensity of glare source increases, the subjective glare experience (as measured by modified De Boer scale) will also increase.

Table 20: Descriptive ANOVA Summary Table for Hypothesis 4

\begin{tabular}{lllll}
\hline Variable & Dim & Mod & Bright & P-value \\
\hline * Rating - Neuroticism Data & 4.19444 & 5.16667 & 6.69444 & 0.0016 \\
& $(1.88757)$ & $(1.97073)$ & $(1.87976)$ & \\
$*$ Rating - Extraversion Data & 3.33333 & 4.97222 & 6.58333 & $<.0001$ \\
& $(1.50489)$ & $(2.11070)$ & $(1.84112)$ & \\
\hline
\end{tabular}

Table 21: Bivariate Regression Analysis Summary Table for Hypothesis 4

\begin{tabular}{lll}
\hline Variable & $\mathrm{R}^{2}$ & P-value \\
\hline * Illumination and Rating - Neuroticism Data & 0.2437 & 0.0001 \\
* Illumination and Rating - Extraversion Data & 0.2952 & $<.0001$ \\
\hline
\end{tabular}

\subsubsection{Glare Level Main Effect on Rating Response - N Data}

The neuroticism result indicated statistically significant differences rating responses $\left(F_{2,48}=7.4019, p=0.0016\right)$ among glare levels. In general, higher glare level resulted in higher rating or more discomfort (Table 22, Figure 22). The Tukey HSD test indicated that this difference comes from rating response between dim and bright glare level only. Similarly, the Friedman Test was used to test the glare level main effect. Using this non-parametric approach, the results also indicated statistical significant.

Table 22: Descriptive Summary of Rating on Glare Level Main Effect - N Data

\begin{tabular}{lll}
\hline Glare Level & Rating & Std. Dev. \\
\hline Dim & 4.19444 & 1.88757 \\
Mod & 5.16667 & 1.97073 \\
Bright & 6.69444 & 1.87976 \\
\hline
\end{tabular}




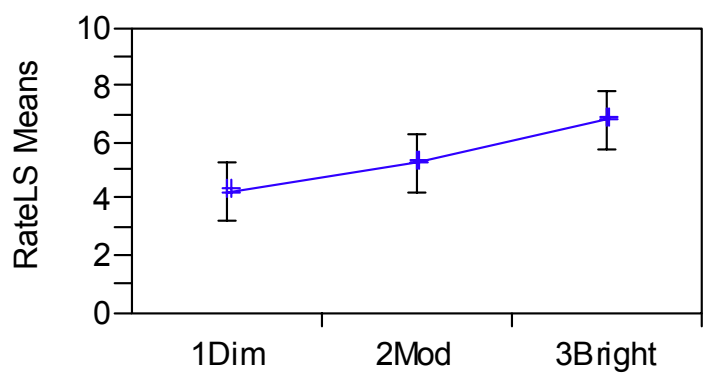

Glare Level

Figure 22: Glare Level Main Effect on Rating - N Data

Moreover, the bivariate regression analysis between measured illumination level and rating responses was analyzed. The regression model of this fit, Rating $=4.1145178$ $+(0.0210743 x$ Illumination $)$, indicated statistically different from the simple mean model $\left(F_{1,52}=16.7519, p=.00001\right)($ Table 23$)$. Note that $\mathrm{R}^{2}=0.243657$ indicated that the fit model can explain 24.37 percent of variance.

Table 23: Analysis of Variance for Significant Linear Relationship between Measured Illumination and Rating - N Data

\begin{tabular}{|c|c|c|c|c|c|}
\hline Source & DF & Sum of Squares & Mean Square & F Ratio & Prob $>F$ \\
\hline Model & 1 & 59.40720 & 59.4072 & 16.7519 & 0.0001 \\
\hline Error & 52 & 184.40761 & 3.5463 & & \\
\hline C. Total & 53 & 243.81481 & & & \\
\hline
\end{tabular}

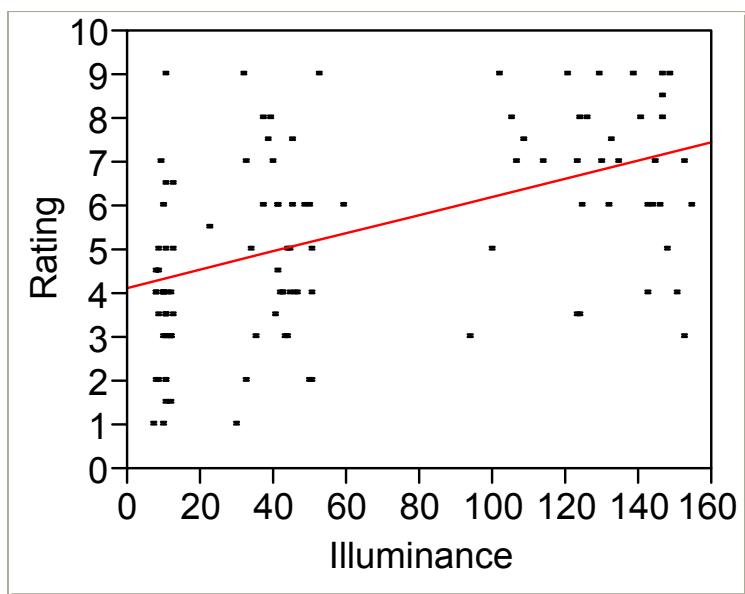

Linear Fit

Figure 23: Scatter Plot between Measured Illuminance and CSI - N Data 


\subsubsection{Glare Level Main Effect on Rating Response - E Data}

The extraversion result indicated statistically significant differences in rating responses $\left(F_{2,48}=24.6211, p=<.0001\right)$ among glare levels. In general, higher glare level resulted in higher rating or more discomfort (Table 24, Figure 24). The Tukey HSD test indicated that the difference comes from the moderate and bright glare levels only. Similarly, the Friedman test was used to test the glare level main effect. The results also indicated statistical significant.

Table 24: Descriptive Summary of Rating on Glare Level Main Effect - E Data

\begin{tabular}{lrr}
\hline Glare Level & Rating & Std. Dev. \\
\hline Dim & 3.33333 & 1.50489 \\
Mod & 4.97222 & 2.11070 \\
Bright & 6.58333 & 1.84112 \\
\hline
\end{tabular}

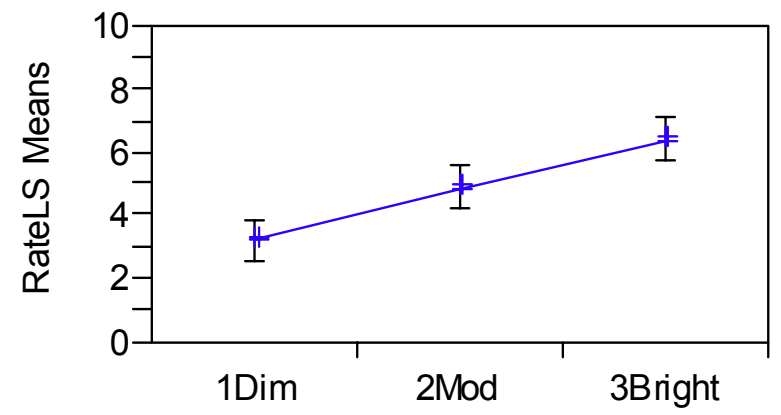

Glare Level

\section{Figure 24: Glare Level Main Effect on Rating - E Data}

The bivariate regression analysis between measured illumination level and rating was analyzed. The regression model of this fit, Rating $=3.5465522+(0.0217594 x$ Illumination), indicated statistically different from the simple means model $\left(F_{1,52}=\right.$ $21.7808, \mathrm{p}<.0001)($ Table 25$)$. Note that $\mathrm{R}^{2}=0.29521$ indicated that the fit model can explain 29.52 percent of variance. 
Table 25: Analysis of Variance for Significant Linear Relationship between Measured Illumination and Rating - E Data

\begin{tabular}{|c|c|c|c|c|c|}
\hline Source & $\mathrm{DF}$ & Sum of Squares & Mean Square & F Ratio & Prob $>F$ \\
\hline Model & 1 & 78.79903 & 78.7990 & 21.7808 & $<.0001$ \\
\hline Error & 52 & 188.12690 & 3.6178 & & \\
\hline C. Total & 53 & 266.92593 & & & \\
\hline
\end{tabular}

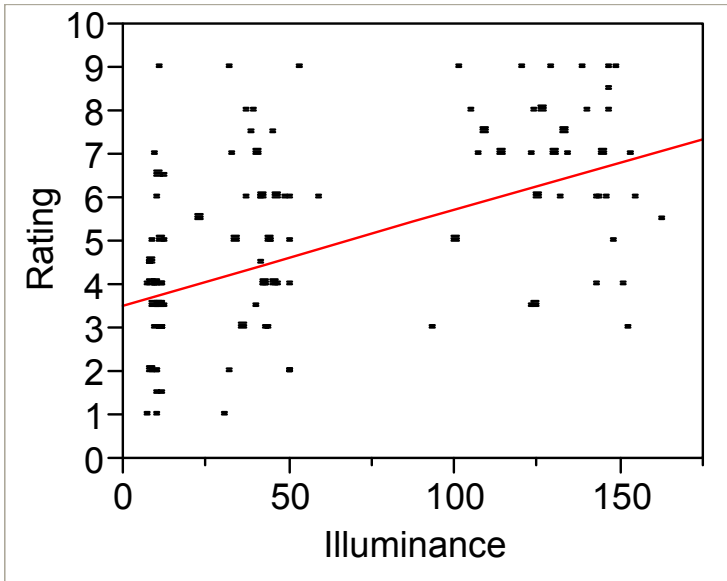

Linear Fit

Figure 25: Scatter Plot between Neuroticism Score and Average Rating - E Data

In conclusion, Hypothesis 4 was supported by both neuroticism and extraversion data and also by using both ANOVA and bivariate regression tests (i.e., significant differences of rating responses between high and low score trait groups, strong positive relationship between measured illuminance and rating responses).

\subsubsection{Hypothesis 4 - Discussions}

Theeuwes (2002) indicated that individual's rating responses are dependent upon the glare illuminance at the observer's eyes. He found that the higher illumination resulted in higher glare rating responses. Similarly, results from the current study indicated that individuals' given ratings were significantly related to the glare intensity levels, and the illumination level at participant's eyes. This finding was supported by the data from both neuroticism and extraversion groups, and also from both parametric and non-parametric statistics (i.e., significant differences among all glare levels; the greater the intensity of the glare source, the greater the increase in the subjective rating). 
Note that the glare discomfort rating was found to be dependent upon other factors as well. Such factors include glare source size (Alferick, 1996, Sivak, Simmons, \& Flannagan, 1990), glare intensity (Bullough, 1999, 2002), color of light source (Bullough, 1999), difficulty of the task to be performed under glare exposure (Sivak et al., 1991), and previous exposure to glare situations (Sivak et al., 1991). In this study, most of above factors were controlled by experimental settings (i.e., using the HID headlamp as glare source with fixed enclosure, having participants perform the same task). Additionally, the factor on past experience with glare exposure was expected to be controlled by including only participants who are students at the Virginia Tech, or residents in nearby areas as they would be expected to have quite similar exposure experienced from driving and living in the same geographical area. On the other hand, the glare source intensity was varied to manipulate three glare levels. Therefore, the change in subjective response is expected to directly relate to the intensity of the glare source only.

\subsubsection{Hypothesis 5}

Participants with high neuroticism scores will report higher subjective glare experience (as measured by modified De Boer scale) compared to people with low neuroticism scores.

Table 26: Descriptive ANOVA Summary Table for Hypothesis 5

\begin{tabular}{llll}
\hline Variable & High N & Low N & P-value \\
\hline Rating - Neuroticism Data & 5.50000 & 5.20370 & 0.5822 \\
& $(2.59066)$ & $(1.61876)$ & \\
\hline
\end{tabular}

Table 27: Bivariate Regression Analysis Summary Table for Hypothesis 5

\begin{tabular}{lll}
\hline Variable & $\mathrm{R}^{2}$ & $\mathrm{P}$-value \\
\hline Neuroticism Score and Rating & 0.0365 & 0.4473 \\
\hline
\end{tabular}




\subsubsection{Neuroticism Main Effect on Rating Response}

The result indicated no statistically significant differences $\left(F_{1,48}=0.3069, p=\right.$ 0.5822 ) between the two neuroticism score groups. Similarly, the Kolmogorov-Smirnov method was used to test the neuroticism type main effect. Using this parametric approach, the results indicated no statistical significant. In general, the high neuroticism scorer gave slightly higher rating responses than the low scorers. However, the high scorer also reported more variation (Figure 26).

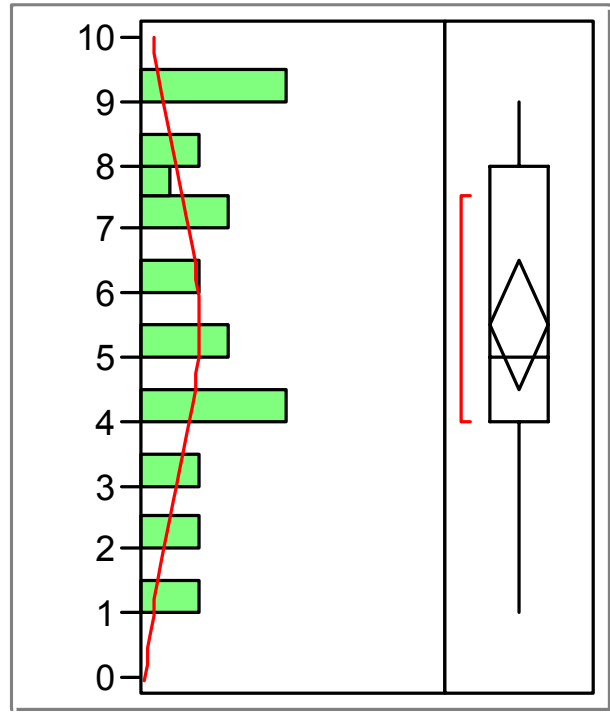

High N: Normal $(5.5,2.59066)$

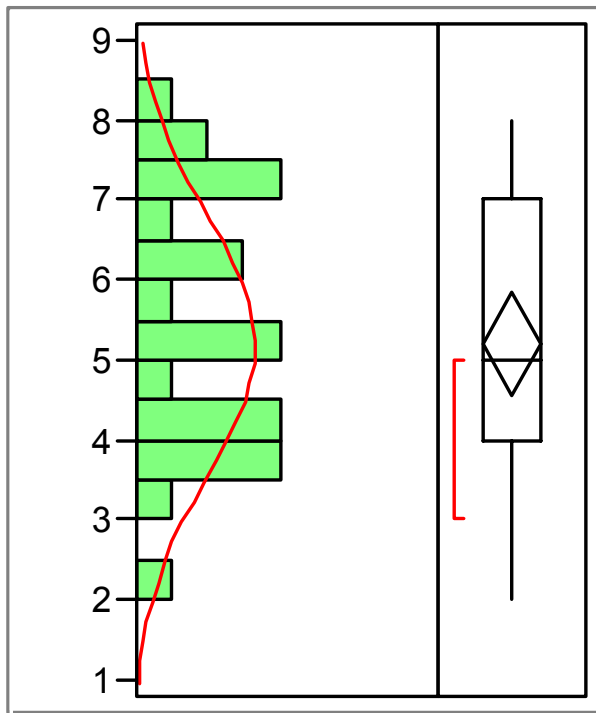

Low N: Normal $(5.2037,1.61876)$

Figure 26: Frequency Distribution of Average Rating Responses from High and

\section{Low Neuroticism Scorer Groups}

It is expected that the high variability of rating response in the high neuroticism scorer group is the major reason for the insignificant differences between the two groups. As shown in Figure 26, the high neuroticism scorer group reported rating ranging from 1.0 to 9.0 while the low scorer group gave rating in the range from 2.0 to 8.0. Additionally, the rating of high scorer group indicated with less likely to be a normal distribution as well.

The bivariate regression analysis between neuroticism scores and average rating responses given by that individual was performed. The regression model of this fit, 
Rating $=4.2064896+(0.0255155 \times \mathrm{N}$-Score $)$, indicated no statistically different from the simple mean model $\left(F_{1,16}=0.6069, p=0.4473\right)($ Table 28$)$. Note that $\mathrm{R}^{2}=0.0365$ indicated that the fit model can explain 3.65 percent of variance.

Table 28: Analysis of Variance for Significant Linear Relationship between Neuroticism Score and Average Rating

\begin{tabular}{llllll}
\hline Source & DF & Sum of Squares & Mean Square & F Ratio & Prob $>$ F \\
\hline Model & 1 & 1.906102 & 1.90610 & 0.6069 & 0.4473 \\
Error & 16 & 50.254392 & 3.14090 & & \\
C. Total & 17 & 52.160494 & & & \\
\hline
\end{tabular}
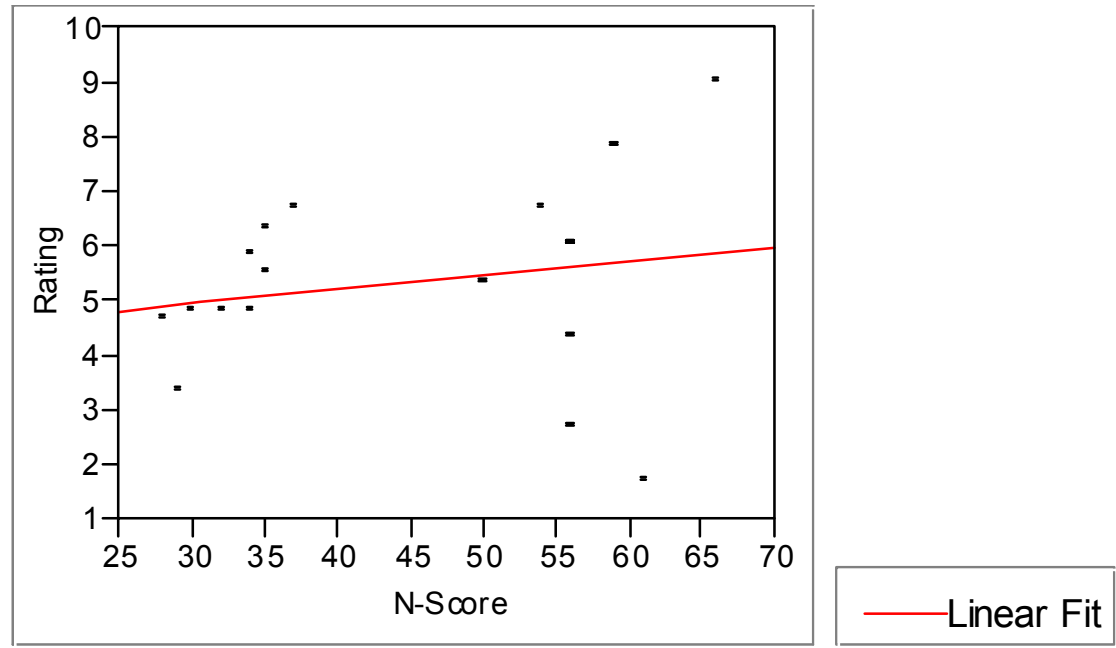

Figure 27: Scatter Plot between Neuroticism Score and Average Rating

In conclusion, Hypothesis 5 was not supported using both ANOVA and bivariate regression tests (i.e., no significant differences of rating responses between high and low neuroticism scorers, low positive relationship between neuroticism score and rating response).

\subsubsection{Hypothesis 5 - Discussions}

A primary objective of this study was to examine the influence of trait based individual differences on the subjective response. This hypothesis anticipated that there 
would be significant differences in subjective responses due to different personality types (i.e., high and low neuroticism scorers) under the same glare conditions.

As previously shown in the results section, no significant difference in rating responses between the high and low $\mathrm{N}$ score groups was found. The high variability of the high score group seems to be the reason for this insignificant finding. Having rapid mood swings and usually emotional, the rating response of the high scorers may be influenced by their mood state at the time of the experiment. This suggestion is supported by findings suggesting possible roles of personality traits and mood states in the processing of emotional information (review by Rusting, 1998). For example, Isen \& Shalker (1982) found that people in a positive mood state gave ratings for presented slides as more pleasant, while those in a negative mood rated them as more unpleasant. For this study, a high $\mathrm{N}$ participant who had just successfully finished an examination prior to the experiment time may be in a very good mood. Therefore, this participant's mood may result in the lower rating reported in this study. At the same time, testing with the broad dimension such as neuroticism has somewhat neglected the more specific negative trait effects (e.g., depression and anxiety), which may give some explanations for these scattered responses. For example, more specific traits of neuroticism (e.g., depression and anxiety) maybe the major reason for subjective rating rather than the neuroticism itself.

Nonetheless, though not significant, the results shown in linear fit (Figure 27) suggested that the high score groups reported relatively higher rating or more discomfort than the low scorers across all glare levels. This tendency is closely related to a recent study (Jang et al., 2002) that reported significant roles for neuroticism and actual performance on self-reported measures of mobility (i.e., lower rating or less mobility in the high $\mathrm{N}$ individual).

The above tendency can be explained by several underlying reasons. Neuroticism is associated with the propensity to experience emotional distress and frequent complaints (Costa \& McCrae, 1992; Eysenck \& Eysenck, 1985). Therefore, a high neuroticism scorer might feel more depressed and report a higher rating than one with a lower score. These propensities are physiologically explained by Larsen \& Ketellar (1991), who proposed that the central nervous system structures, which constitute an 
"anxiety system" are more active in high neuroticism than in low neuroticism individuals. Secondly, neuroticism is found to be significantly related to the trait measures of selffocused attention (Watson \& Pennebaker, 1989), and thus may have greater detection of their physical changes from glare exposure which eventually lead to poorer rating of discomfort similar to the earlier finding of relationship between self focused attention and report of bothersome (Clark \& Watson, 1988). In conclusion, Hypothesis 5 was not supported by current study mainly due to the high variation of rating response given by high neuroticism participants.

\subsubsection{Hypothesis 6}

Participants with high extraversion scores will report lower subjective glare experiences (as measured by modified De Boer scale) compared to people with low extraversion scores.

Table 29: Descriptive ANOVA Summary Table for Hypothesis 6

\begin{tabular}{llll}
\hline Variable & High E & Low E & P-value \\
\hline * Rating - Extraversion Data & 3.77778 & 6.14815 & $<.0001$ \\
& $(1.65444)$ & $(2.14752)$ & \\
\hline
\end{tabular}

Table 30: Bivariate Regression Analysis Summary Table for Hypothesis 6

\begin{tabular}{lll}
\hline Variable & $\mathrm{R}^{2}$ & $\mathrm{P}$-value \\
\hline * Extraversion Score and Rating & 0.5511 & 0.0004 \\
\hline
\end{tabular}

\subsubsection{Extraversion Main Effect on Rating Response}

The extraversion result indicated statistically significant differences $\left(F_{1,48}=\right.$ 39.2902, $p=<.0001$ ) between high and low extraversion score groups. In general, high extraversion scorer gives lower rating or perceived more comfort (Table 31, Figure 28). In addition, the Kolmogorov-Smirnov method was used to test the extraversion type main effect. This non-parametric approach indicated statistical significant as well. 
Table 31: Descriptive Summary of Rating on Extraversion Type Main Effect

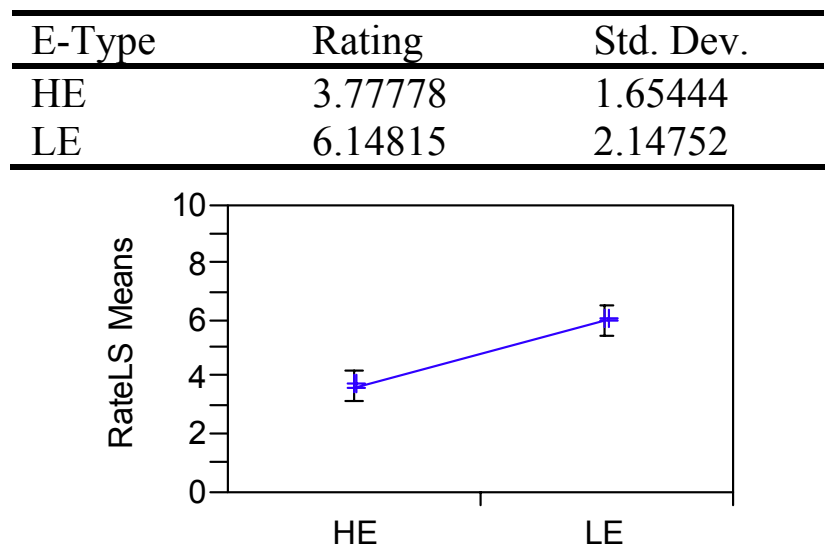

Type

Figure 28: Extraversion Type Main Effect on Rating

On contrary to the neuroticism data, the variability within high extraversion score group was quite small while the one of low score group is larger (Figure 29). As shown in Figure 29, the high extraversion scorer group reported rating ranging from 1.0 to 7.0 while the low scorer group gave rating in the range from 2.0 to 9.0. Note that the rating of high scorer group indicated with less likely to be a normal distribution as well.

Figure 29: Frequency Distribution of Average Rating from High and Low Extraversion Score Groups

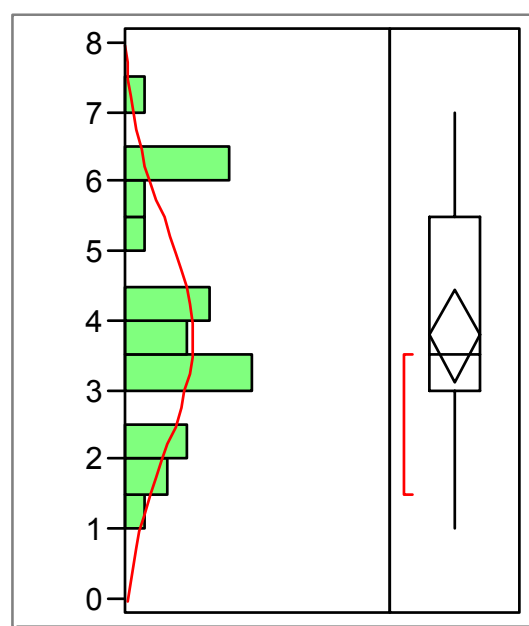

High E: Normal (3.77778,1.6544)

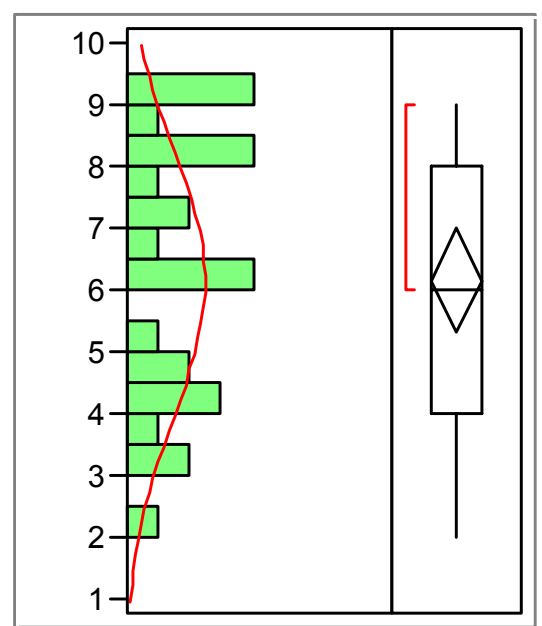

Low E: Normal (6.14815,2.14752) 
Moreover, the bivariate regression analysis between individual's extraversion scores and the average rating given by that individual was performed. The regression model of this fit, Rating $=9.5657348-(0.0670849 \times E$-Score $)$, indicated statistically significant linear relationship $\left(F_{1,16}=19.6453, p=0.0004\right)($ Table 32$)$. Note that $\mathrm{R}^{2}=$ 0.5511 indicated that the model can explain up to 55.11 percent of variance.

Table 32: Analysis of Variance for significant linear relationship

\begin{tabular}{lrrrrr}
\hline Source & DF & Sum of Squares & Mean Square & F Ratio & Prob > F \\
\hline Model & 1 & 25.185423 & 25.1854 & 19.6453 & 0.0004 \\
Error & 16 & 20.512107 & 1.2820 & & \\
C. Total & 17 & 45.697531 & & & \\
\hline
\end{tabular}
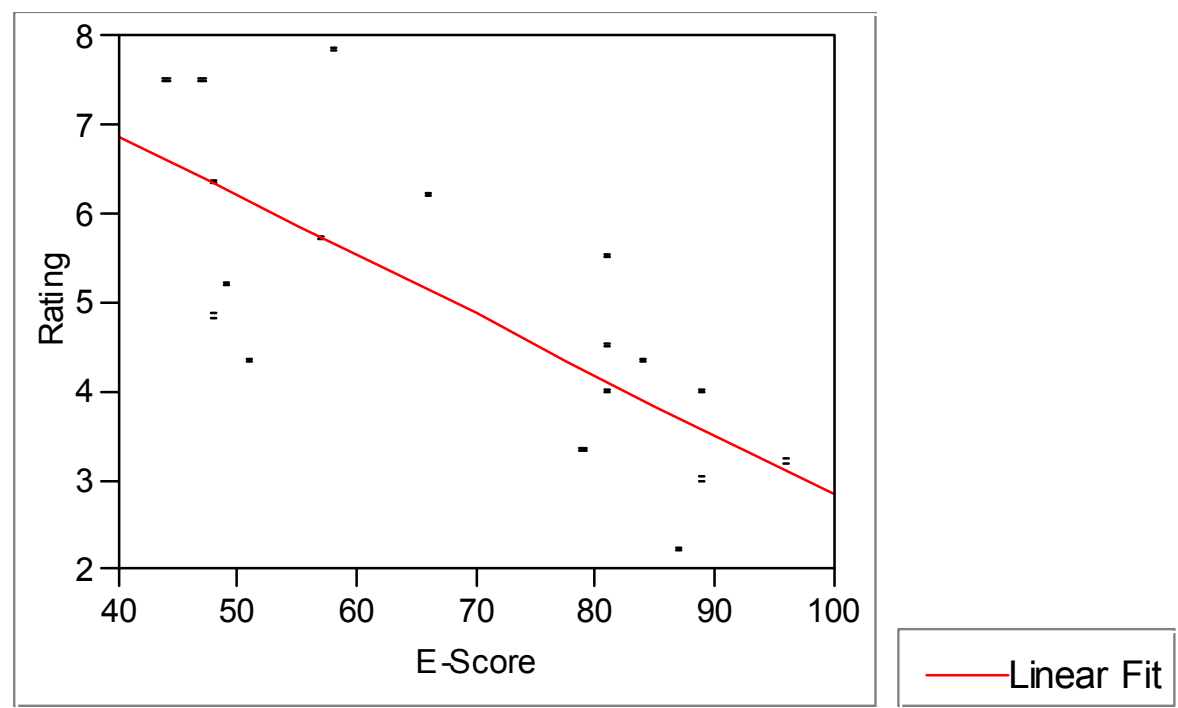

Figure 30: Scatter Plot between Extraversion Score and Average Rating

In conclusion, Hypothesis 6 was supported using both ANOVA and bivariate regression tests (i.e., significant differences of rating responses between extraversion scorers, high negative relationship between extraversion score and rating response).

\subsubsection{Hypothesis 6 - Discussions}

Results from this study supported this hypothesis by showing significant differences in rating responses between the two groups using both parametric and non- 
parametric statistics. The results also showed that the high extraversion score group rated the glare situations as more comfortable than the low scorer. Note that this similar tendency is present across all three glare levels.

The above finding can be explained using the Eysenck's Arousal Theory (1967). According to this theory, introverts are characterized by lower thresholds of arousal in the reticular activating system (ARAS) as well as by greater physiological reactivity to sensory stimulation than extraverts (e.g., more salivate responses to the lemon drop test by Eysenck \& Eysenck, 1967; on nicotine stimulus by Gilbert \& Hagen, 1985; enhanced auditory evoked potential responses to moderate intensity tones by Stelmack et al., 1977; 1990; and on auditory by Wigglesworth \& Smith, 1976). This higher response to stimulation reflects the activity of protective, inhibitory process of introverts in order to avoid the high intensity stimulus situation and suggests that introverts prefer situations with lower stimulus intensity. The introvert's preference is found repeatedly in prior studies. For example, Campbell \& Hawley (1982) found that introverts often chose to study alone where the external stimulations are minimized. Green (1984) found that male introverts chose less intense noise levels to be heard during a paired-associates (PA) learning task than male extraverts. In addition to this ASRS system, other findings suggest higher pain threshold in extraverts (e.g., Dunstone et al., 1964; Haslam, 1966). These findings further suggested that manipulated glare directly pointed toward the observer's eyes could eventually lead to perceived pain.

Furthermore, the differences in coping behavior used by low and high scorers can also be used to explain the finding of extraversion effects on glare rating responses. According to Byrne (1964), a variety of scales for determining extraversion-introversion dimension are correlated with the repression-sensitization defense mechanisms. Typically, introverts tend to be sensitizers, meaning that they are very much aware of their negative emotions or problems and even exaggerate them. On the other hand, extraverts tend to have opposite mechanisms as being repressors. They normally deny or prefer not to verbalize their emotional experiences, which may either due to choice or inability to be aware of their internal negative emotions (Morris, 1967). Having these characteristics, the high E group may avoid (or be unaware) of the glare effects and thus report less discomfort to the situation, while the low E used the reverse strategy. 


\subsubsection{Hypothesis 7}

Subjective glare experience (as measured by modified De Boer scale) will have low relationship with the measured contrast sensitivity index.

Table 33: Bivariate Regression Analysis Summary Table for Hypothesis 7

\begin{tabular}{lll}
\hline Variable & $\mathrm{R}^{2}$ & $\mathrm{P}$-value \\
\hline * CSI and Rating - Neuroticism Data & 0.08589 & 0.0315 \\
* CSI and Rating - Extraversion Data & 0.2198 & 0.0002 \\
* CS and Rating - Neuroticism Data & 0.13149 & 0.0070 \\
* CS and Rating - Extraversion Data & 0.16079 & 0.0027 \\
\hline
\end{tabular}

\subsubsection{Relationship between CSI and Rating Response - N Data}

The bivariate regression analysis between two dependent measures for the neuroticism data was performed. The regression model of this fit, $C S I=1.6834381+$ (0.1875521 $x$ Rate), indicated statistically significant linear relationship $\left(F_{1,52}=4.8861, p\right.$ $=0.0315)$ (Table 34). However, $\mathrm{R}^{2}=0.08589$ indicated that the fit model can explain 8.59 percent of variance.

Table 34: Analysis of Variance for Significant Linear Relationship between Rating and CSI - N Data

\begin{tabular}{llllll}
\hline Source & DF & Sum of Squares & Mean Square & F Ratio & Prob $>$ F \\
\hline Model & 1 & 8.576376 & 8.57638 & 4.8861 & 0.0315 \\
Error & 52 & 91.274234 & 1.75527 & & \\
C. Total & 53 & 99.850610 & & & \\
\hline
\end{tabular}




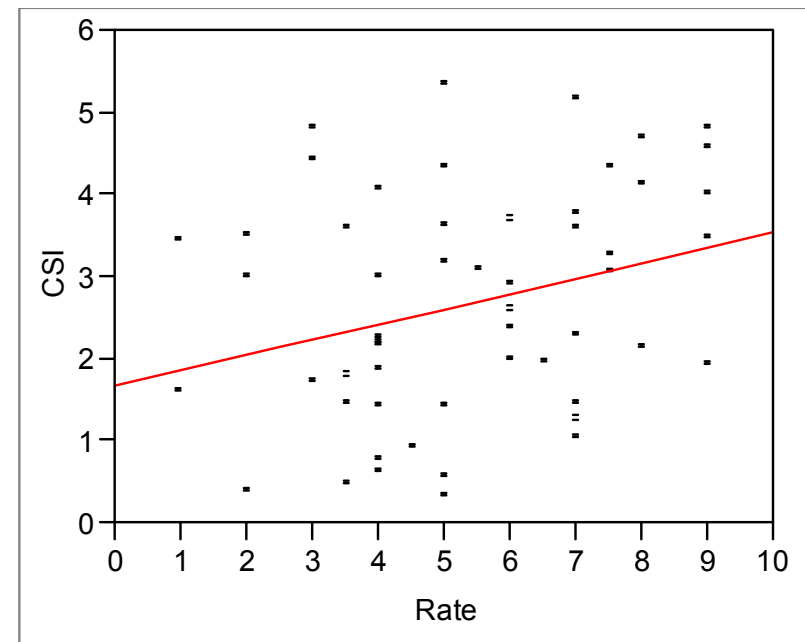

Linear Fit

Figure 31: Scatter Plot between Rating and CSI - N Data

\subsubsection{Relationship between CSI and Rating Response - E Data}

The bivariate analysis between two dependent measures for the extraversion data was performed. The regression model of this fit, $C S I=1.1112599+(0.3118672 x$ Rating), indicated statistically significant linear relationship $\left(F_{1,52}=15.9342, p=\right.$ 0.0002) (Table 35). However, $\mathrm{R}^{2}=0.2198$ indicated that the model can explain 21.98 percent of variance.

Table 35: Analysis of Variance for Significant Linear Relationship between Rating and CSI - E Data

\begin{tabular}{lrrrrr}
\hline Source & DF & Sum of Squares & Mean Square & F Ratio & Prob $>$ F \\
\hline Model & 1 & 25.96152 & 25.9615 & 15.9342 & 0.0002 \\
Error & 52 & 84.72361 & 1.6293 & & \\
C. Total & 53 & 110.68513 & & & \\
\hline
\end{tabular}




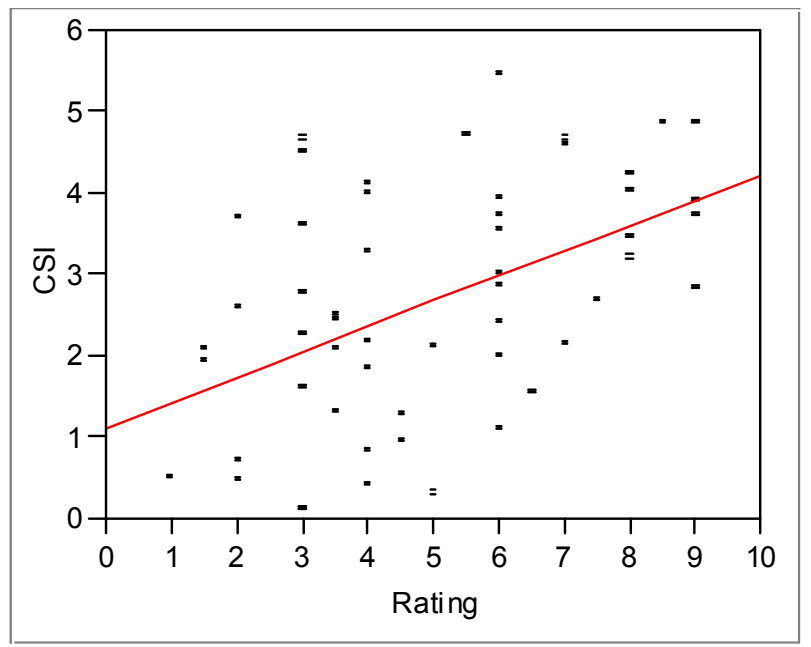

Linear Fit

Figure 32: Scatter Plot between Rating and CSI - E Data

\subsubsection{Relationship between CS and Rating Response - N Data}

The bivariate regression analysis between two dependent measures for the neuroticism data was performed. The regression model of this fit, $C S=4.9547249$ (0.2342887 $x$ Rate), indicated statistically significant linear relationship $\left(F_{1,52}=7.8724, p\right.$ $=0.007)$ (Table 36). However, $\mathrm{R}^{2}=0.13149$ indicated that the fit model can explain 13.15 percent of variance.

Table 36: Analysis of Variance for Significant Linear Relationship between Rating and CS - N Data

\begin{tabular}{lrrrrr}
\hline Source & DF & Sum of Squares & Mean Square & F Ratio & Prob $>$ F \\
\hline Model & 1 & 13.38328 & 13.3833 & 7.8724 & 0.0070 \\
Error & 52 & 88.40170 & 1.7000 & & \\
C. Total & 53 & 101.78498 & & & \\
\hline
\end{tabular}




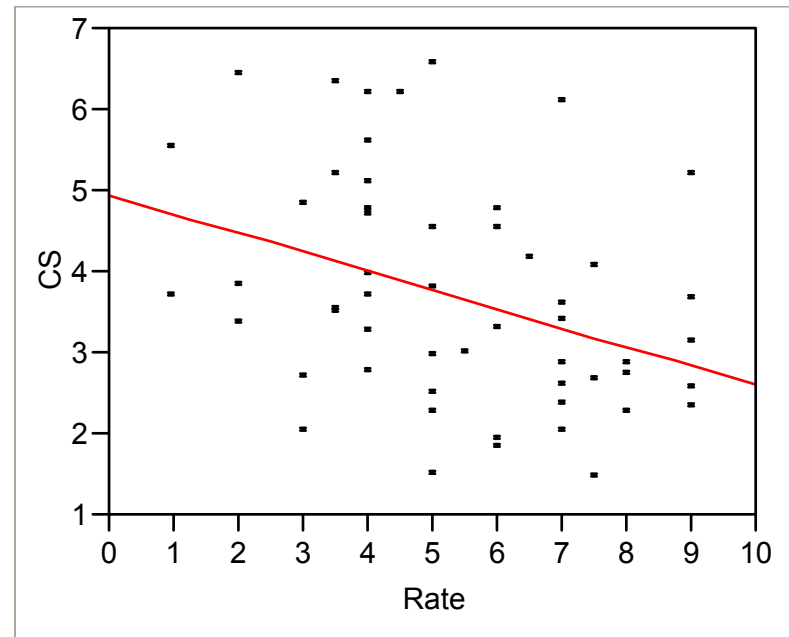

Linear Fit

Figure 33: Scatter Plot between Rating and CS - N Data

\subsubsection{Relationship between CS and Rating Response - E Data}

The bivariate regression analysis between two dependent measures for the extraversion data was performed. The regression model of this fit, $C S=4.8482833$ (0.2425634 $x$ Rating) indicated statistically significant linear relationship $\left(F_{1,52}=9.9630\right.$, $p=0.0027$ ) (Table 37). However, $\mathrm{R}^{2}=0.16079$ indicated that the model can explain 16.08 percent of variance.

Table 37: Analysis of Variance for Significant Linear Relationship between Rating and CS - E Data

\begin{tabular}{lrrrrr}
\hline Source & DF & Sum of Squares & Mean Square & F Ratio & Prob $>$ F \\
\hline Model & 1 & 15.705120 & 15.7051 & 9.9630 & 0.0027 \\
Error & 52 & 81.969529 & 1.5763 & & \\
C. Total & 53 & 97.674650 & & & \\
\hline
\end{tabular}




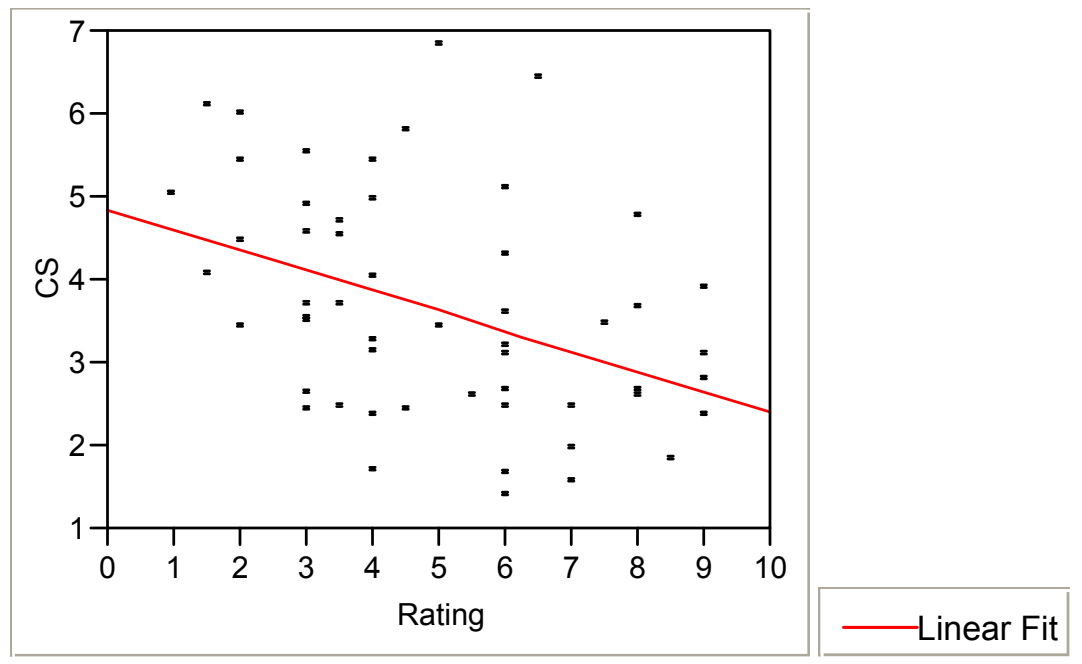

Figure 34: Scatter Plot between Rating and CS - E Data

In conclusion, Hypothesis 7 was supported particularly the CS data (i.e., low relationship between CS and rating response for both neuroticism and extraversion data). On the other hand, the low relationship between CSI and rating response was found only on neuroticism data while the extraversion data showed to have slight relationship.

\subsubsection{Hypothesis 7 - Discussions}

It was hypothesized that there will be low correlation between glare subjective responses and the measured CSI. This hypothesis was supported by the low variance explained by these relationships, particularly in the neuroticism data $(8.59 \%$ for CSI and $13.15 \%$ for CS). However, although the relationship was not strong, results from both neuroticism and extraversion data showed the expected direction of the correlations between the subjective responses and actual performances (i.e., positive relationship between CSI and rating and negative relationship between CS and rating).

Two major factors were expected to be the underlying reasons for the above relationships: personality type and age. Personality type was found to increase variance in the rating response (refer to Hypotheses 5 and 6), while the age factor showed increased variances in visual performance (refer to the secondary analysis). This means that, personality and age factors can indirectly influence the relationship between actual 
performance and rating responses. Thus, parsing the age and personality factors (partial model) would result in higher explained variance (i.e., $\mathrm{R}^{2}$ ). These parsing results were shown to be as expected (refer to the secondary analysis result section). Nonetheless, the parsing results may be confounded by lower number of data points as well (i.e., with fewer data, the lower variance leads to higher explained variance).

\subsection{Summary of Results}

Results of the present study are summarized in the following section. First, findings regarding glare intensity, and differences involving individuals' visual performances (CSI, CS) and subjective responses are addressed. Similarly, the effects of trait-based individual differences (i.e., neuroticism and extraversion traits) on above dependent measures are reviewed further. Following this, the relationships among dependent measure from both screening and glare test sessions are examined. Finally, the analyses including the factor of gender and age related differences are concluded.

Glare intensity levels as measured in illumination was a very important for influencing the ultimate outcomes of both visual performances (CSI, CS) and subjective responses. Results indicated significant differences of CSI and CS among glare intensity levels for both neuroticism and extraversion data. The bivariate analysis indicated that as glare intensity increased, CSI increased and CS decreased. Thus, Hypotheses 1 was supported. Furthermore, significant difference on rating response was also found with the glare intensity variable on both neuroticism and extraversion data. The bivariate analysis indicated that as glare intensity increased, rating response also increased. Therefore, Hypothesis 4 was also supported

On the other hand, considering the trait factor, no significant difference on visual performance (both CSI and CS) under glare conditions was found. This conclusion was found on both neuroticism and extraversion data. Thus, Hypothesis 2 and 3 were supported by the current findings. However, though the actual performances were not significantly different among trait score groups, the trait factor did affect individuals' rating responses particularly the extraversion score factor. This finding thus supported Hypothesis 6 . The bivariate regression test also indicated that as extraversion score 
increased, rating response decreased. Adversely, Hypothesis 5 was not supported due to insignificant rating responses between high and low neuroticism scorers. In addition, the bivariate analysis between individuals' neuroticism score and rating response indicated low relationship. Generally, the high neuroticism scorer reported slightly higher rating response than the low scorer.

As hypothesized, the bivariate regression analysis indicated low relationship between subjective rating and actual performances particularly on CS for both neuroticism and extraversion data. Nonetheless, the relationship between rating response and CSI of extraversion data showed to be somewhat strong, while the one of neuroticism data was low. In general, except extraversion on CS, higher rating was related to the lower performances (i.e., higher CSI and lower CS). 


\subsection{CONCLUSIONS}

\subsection{Concluded Model}

The suggested model can be drawn from all significant relationships as shown in the following diagram (Figure 35). Glare intensity is found to affect all dependent measures including the subjective rating, CSI, and CS. Personality type (mainly extraversion) was the moderator between glare intensity and rating response, while the visual performance (CSI and CS) gained no influence from this trait factor. Age was a factor affecting CS but not both CSI and rating response. Finally, because rating response and visual performance are affected by different factors, there were low relationships between the rating response and both measured CSI and CS.

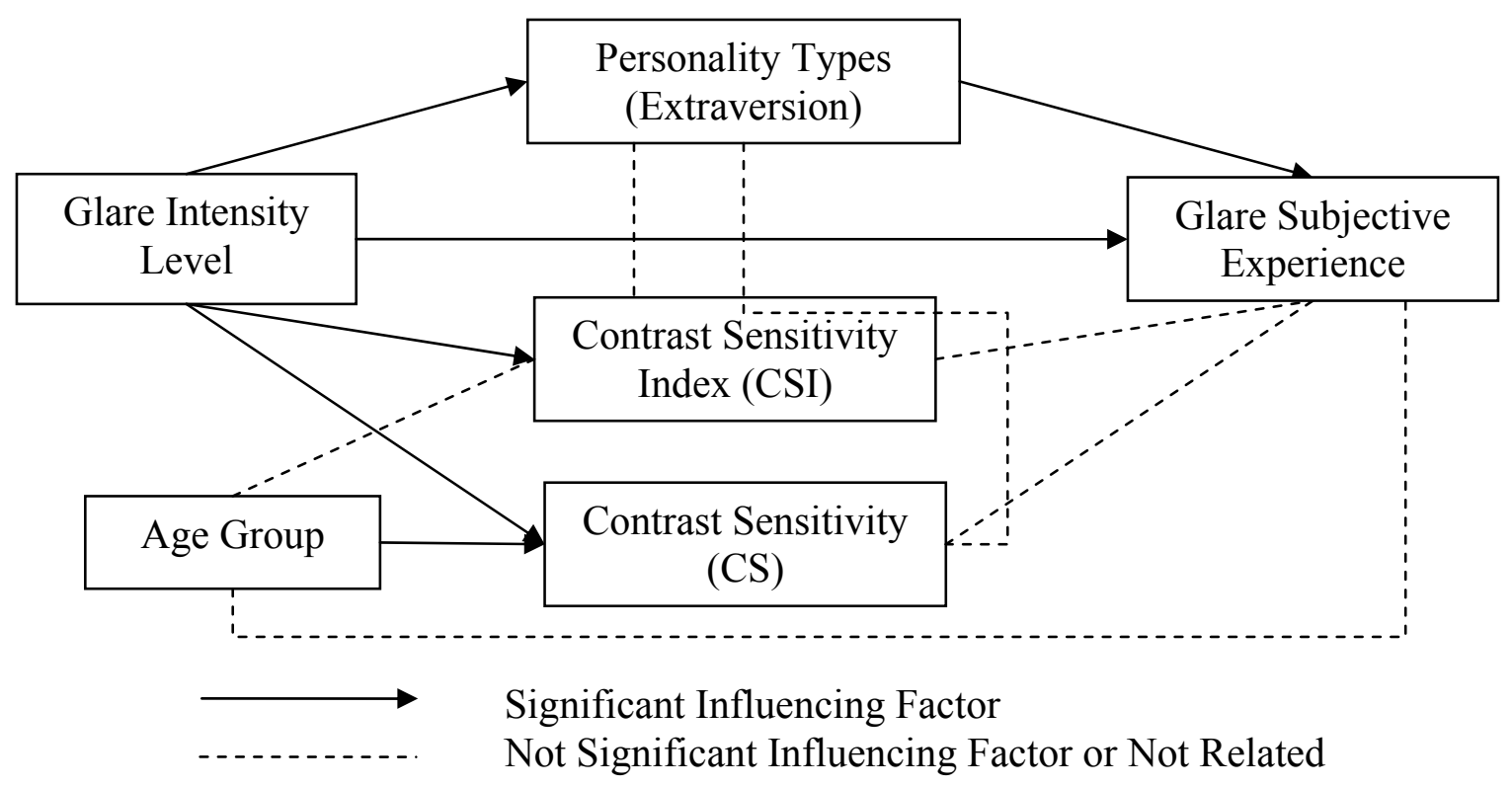

Figure 35: Concluded Model of Discomfort Glare Subjective Experience and Visual Performance in Glare Situation 


\subsection{Application of results}

\section{$\underline{\text { Measurement of glare discomfort and its interpretation }}$}

One of the most significant applications of this study is toward possible interpretations of earlier findings. According to earlier studies, high variation among subjective responses for glare discomfort has been found repeatedly (Berman et al., 1994; Theeuwes, 2002). Participants' differences in terms of extraversion trait may be an underlying reason for these variations. Also, the low correlation between rating response and actual performance may be similarly explained by the findings of this study.

Moreover, the other application is toward conducting future studies to generalize these results. For instance, if the results of a study will be applied to a wide group of people such as drivers, the participants to be included in the study should be a wide range of extraversion scorers as well. To achieve this purpose the screening test on personality questionnaire should be conducted. This screening becomes crucial as the prior findings indicated that volunteers for the general experiments are more likely to be the high extraversion scorers due to their out going style (McLaughlin \& Harrison, 1974). Thus, with no screening, the high scorer will be generalized to the low scorer, which is not appropriate. Finally, as researchers have attempted to find some other objective measures to evaluate the discomfort glare, the sensitivity to trait individual differences may serve as another criterion to evaluate any future techniques.

\section{$\underline{\text { Selection and Training of Drivers/Workers }}$}

According to the differences in perceived discomfort among individuals in different traits and ages, several recommendations are made for the selection of personnel to work under glare exposure. The high extraversion and the low neuroticism individuals are more appropriate to work under high glare intensity, because these two groups are associated with the tendency to perceive less discomfort. Moreover, as the actual performances (CS and CSI) are not significantly different between trait groups, both low extraversion and high neuroticism may be selected as necessary. However, additional 
training could be conducted to increase familiarization and reduce perceived discomfort in the glare events (Sivak et al., 1991).

\subsection{Limitation of the Study}

The expected limitations in this study are listed and discussed in the following subsection.

\subsubsection{Sample Size}

One of the very critical limitations in this study is the small sample size. The total of thirty-six individuals is very small as compared to other personality studies. For example, to investigate only the neuroticism trait, Jang et al., (2002) used a sample size of 459 elderly individuals to find significant differences in their self-report on mobility. The small sample size may prevent the neuroticism trait effect from reaching statistical significance in this study. For example, the very low rating response in high neuroticism group may be considered as the outliers and may not affect the statistical analysis, if more participants were used. Furthermore, the pool of potential participants from which high and low trait individuals were selected might be too small to produce meaningfully different group discriminations. Selection of thirty-six participants from a pool of seventy-eight individuals may not different enough to produce significant differences in the study among trait score groups. Additionally, a greater number of participants could further affect the investigation of the relationship between personality traits (both neuroticism and extraversion) and actual performance.

Various findings related to high and low neuroticism score individuals suggest several reasons for poorer performance in the former group (e.g., overreaction to the stressful stimuli, Eysenck \& Eysenck, 1975; more distractible by the threatening stimuli, Wallace \& Newman, 1998; less motivation to perform task, Judge \& Ilies, 2002; Slater, 1944 cited in Eysenck, 1998). On the other hand, a range of studies suggested several reasons for lower sensory threshold of the low extraversion scorer as compared to the high extraversion scorer (e.g., Smith, 1968, in auditory stimuli). However, when the glare source intensity increases the opposite result is expected. The low extraversion 
scorer could have lower performance due to higher annoyance from glare exposure (refer to ASRS theory by Eysneck, 1967). Note that considering only young participant data, the relationships of visual performances (CSI and CS) and trait groups also similarly suggested the above propensities.

\subsubsection{Reliability of Questionnaire Response}

Additionally, some limitations may be the consequence of reliability of the personality questionnaire answers of the highly neurotic individual. According to the review by Eysenck (1998), neurotics are show to have less test-retest reliability than normal group. The neuroticism personality trait was found to have scattered characteristics and which may lead to lower validity compared to the extraversion trait.

\subsubsection{Experimental Design}

Considering the design of experiment, the unrealistic event used in this study is another limitation for generalization of the results. In actual circumstances, the glare exposure is more likely to have an intermittent pattern rather than to be constant. Furthermore, the visual task performed in this test (contrast sensitivity test using the Landolt's $C$ target) is considered much easier than many of the actual tasks expected to found in the glare situations (e.g., driving at night). Having such difference, the effects of inappropriate responses such as false alarm of the detectable responses are much less critical as compared to the realistic case (i.e., able to see the Landolt's target is much less critical than able to see the pedestrian). Therefore, while the low E participant (the careful and scrupulous person) may tend to wait for the definite clear target, the high $\mathrm{E}$ may take guess on the answer. This expectation is supported by the tendency to be risk takers in extraverts (Cameron \& Myers, 1965) and the more frequent for extraverts to guess and get more false alarm response (Berbner \& Flavell, 1978). Lastly, the fact that this study did not measure participant's mood states has neglected the possible important of a confounding effect, the individual's mood, on rating response. 


\subsection{Future Research}

Future studies should be conducted using more number of participants in order to reach statistically significant results. With more participants, the tendency found in this present study may become statistically meaningful. The expected tendencies include the neuroticism trait main effect on rating response and, with less possibility, the differences in actual performance due to both individual traits. For future study, the present results can be used as an appropriate pilot test to calculate number of expected participants.

Secondly, more realistic experimental conditions should be simulated. For example, to generalize the results to the driving task, the simulation of driving tasks and intermittent headlight glare exposure should be used. With more realistic set up, the increase of external validity is expected. Also, the expected problems with motivation and risk taking can be reduced. However, to check this possible confounding effect, the false alarm responses should be collected. With more realistic tasks, the rating response was turn out to be even less correlated to the actual performance as found in the earlier study using actual driving tests (Theeuwes, 2002)

Not only the measures of the performance on actual tasks but, the discomfort levels should also be measured using more objective methods if possible (e.g., evaluating both EMG as well as the subjective rating). Additional measurement of dependent variables could be used to compare the possible relationship among them, and to validate the effect of trait-based individual differences on perceived discomfort measured by various approaches. Furthermore, because mood states can be another confounding variable, measure of individuals' moods should be made and later used as covariate variable in result analysis. Likewise, the neutral mood may be induced using some available methods (e.g., reading a list of statements).

Finally, other personality dimensions as well as the lower level trait, especially for neuroticism (e.g., depression and anxiety), should be tested to investigate other possible relationships. Note that age and probably gender factors should be removed from the study to extensively investigate the trait factor. The removal of age variable will be particularly crucial to investigate the main trait effects on actual performances, as age seems to give more influence to the performance rather than the trait itself. 


\section{REFERENCE}

Alferdinck, J.W.A.M. (1991). Discomfort Glare from D1 Headlamps of Different Size. TNO Human Factors Research Institute, Soesterberg, Netherlands.

Allport, G.W. (1937). Personality: A Psychological Interpretation. New York.

Arthur, W. Jr. \& Graziano, W. G. (1996). The Five-Factor Model, Conscientiousness, and Driving Acccident Involvement. Journal of Personality, 64, 593 - 618.

Bailey, I.L, Bullimore, M.A., Raasch, T.W., \& Taylor, H.R. (1991). Clinical Grading and the Effects of Scaling. Investigative Ophthalmology and Visual Science, 32(2), 422-432.

Ball, K.K., Owsley, C., Sloane, M.E., Roenker, D.L., \& Bruni, J.R. (1993). Visual Attention Problems as a Predictor of Vehicle Crashes in Older Drivers. Investigative Ophthalmology and Visual Science, 34(11), 3110-3123.

Beckman, C., Scott, R., \& Garner, L.F. (1992). Comparison of Three Methods of Evaluating Glare. Acta Ophthalmologica, 70(1), 53-59.

Beirness, D. J. (1993). Do We Really Drive as We Live? The Role of Personality Factors in Road Crashes. Alcohol, Drugs and Driving, 9, $129-143$.

Bennett, C. (1977). "Discomfort Glare: Concentrated Sources Parametric Study of Angularly Small Sources. Journal of the Illuminating Engineering Society, 7 (1), 2-14.

Berbner, J. \& Flavell, R. (1978). The Effect of Catch Trials on Speed and Accuracy among Introverts and Extroverts in a Simple RT Task. British Journal of Psychology, 69, 9-15.

Berman, S. M., Bullimore, M. A., Jacobs, R. J., Bailey, I. L., \& Gandhi, N. (1994). An Objective Measure of Discomfort Glare. Journal of the Illuminating Engineering Society, Summer 1994, 40-49.

Buchanan, T. (2001). Online Implementation of an IPIP Five Factor Personality Inventory. University of Westminster. Retreived October, 2002, from http://users.wmin.ac.uk/ buchant/wwwffi/introduction.html.

Bullough J. D. (1999). Effects of Headlamp Color on Visual Perception through Perturbed Atmospheres (M.S. Thesis). Rensselaer Polytechnic Institute, New York.

Bullough J. D et al. (2002). Discomfort and Disability Glare from Halogen and HID Headlamp Systems. Transportation Lighting Group, Lighting Research Center, Rensselaer Polytechnic Institute, New York.

Byrne, D. (1964). Repression-Sensitization as a Dimension of Personality. In B. Maher (Ed.). Progress in Experimental Personality Research, New York: Academic Press, 169-220.

Cameron, B. \& Myers, J.L. (1965). Some Personality Correlates of Risk Taking. Journal of General Psychology, 4, 1-7.

Campbell, J.B., \& Hawley, C.W. (1982). Study Habits and Eysenck's Theory of Extroversion-Introversion. Journal of Research in Personality, 16, 139-146.

Caspi, A. \& Roberts, B. (1999). Personality Continuity and Change across the Life Course. In L.A.Pervin and O.P John (Eds), Handbook of Personality: Theory and Research, New York, 300-326. 
Cellar, D.F., Nelson, Z.C., \& Yorke, C.M. (2000). The Five-Factor Model and Driving Behavior: Personality and Involvement in Vehicular Accidents. Psychological Reports, 86, 454-456.

Clark, L.A., \& Watson, D. (1988). Mood and the Mundane: Relations between Daily Life Events and Self-Reported Mood. Journal of Personality and Social Psychology, 56, 958-964.

Cogan, D.G. \& E.V. Kinsey (1942). The Cornea V. Physiological Aspects. Arch. Ophtalmol., 28, 661-669.

Corso, J.F. (1981). Aging Sensory and Perception. Praeger, New York.

Costa, P.T., Jr. \& McCrae, R.R. (1980). Influence of Extraversion and Neuroticism on Subjective Well-Being: Happy and Unhappy People. Journal of Personality and Social Psychology, 38, 668-678.

Costa Jr., P.T. \& McCrae, R.R., (1992). NEO Personality Inventory (NEO PI-R) and NEO Five Factor Inventory (NEO-FFI): Professional Manual. Psychological Assessment Resources, Odessa, FL.

Costa, P.T., Jr., \& McCrae, R.R. (1994). Stability and Change in Personality from Adolescence through Adulthood. The Developing Structure of Temperament and Personality from Infancy to Adulthood, Hillsdale, NJ: Erlbaum, 139-155.

Craske, D. (1968). A Study of the Relationship between Personality and Accident History. British Journal of Psychology, 41, 399-404.

Davis, D.R., Hockey, G.R., and Taylor, A. (1969). Varied Auditory Stimulation, Temperament Differences, and Vigilance Performance. British Journal of Psychology, 60, 453-457.

Davson, H. (1949). The Physiology of the Eye. Philadelphia: Blakiston Co.

De Boer, J.B. ed. Eindhoven (1967a). Visual Perception in Road Traffic and the Field of Vision of the Motorist. Public Lighting, Netherlands: Philips Technical Library, $1-96$.

De Boer, J. B. (1967). Transaction of the Illuminating Engineering Society. 32, 117.

De Waard, P.W., IJspeert, J.K., Van Den Berg, T.J., \& De Jong, P.T.V.M. (1992). Intraocular Light Scattering in Age-related Cataracts. Investigative Ophthalmology and Visual Science, 33(3), 618-625.

Decina, L.E. \& Staplin, L. (1993). Retrospective Evaluation of Alternative Vision Screening Criteria for Older and Younger Drivers. Accident Analysis and Prevention, 25(3), 267-275.

Derefeldt, G., Lennerstrand, G., \& Lundh, B. (1979). Age Variation in Normal Human Contrast Sensitivity. Acta Ophthalmologica, 57, 679-690.

Domey, R.G., McFarland, R.A., \& Chadwick, E. (1960). Dark Adaptation of a Function of Time II: A Derivation. Journal of Gerontology, 15, 267-279.

Duane, A. (1922). Studies in Monocular and Binocular Accommodation with their Clinical Applications. American Journal of Ophthamol., 5, 865-877.

Duke-Elder, S. (1963). System of Opthalmology. Kimpton, London.

Dunstone, J.J., Dzendolet, G., \& Henckeruth, O., (1964). Effect of some Personality Variables on Electrical Vestibular Stimulation. Perception and Motor Skills, 18, 689-695. 
Eisner, A., Fleming, S.A., Klein, M.L., \& Mauldin, M. (1987). Sensitivity in Older Eyes with Good Acuity: Cross Sectional Norms. Investigative Ophthalmology and Visual Science, 28, 1824-1831.

Eysenck, H.J. (1982). Personality Genetics and Behavior. Praeger, New York.

Eysenck, H. (1998). Dimensions of Personality. Transaction Publishers, New Brunswick and London.

Eysenck, S.B.J. \& Eysenck, J.J. (1967). Salivary Response to Lemon-Juice as a Measure of Introversion. Perceptual and Motor Skills, 24, 1047-1051.

Eyesenck, H.J. \& Eysenck, S.B.G. (1975). Manual of the Eysenck Personality Quetsionnaire. Educational and Industrial Testing Service, San Diego, CA.

Eysenck, H.J. \& Eysenck, M. (1985). Personality and Individual Differences: A Natural Science Approach. Plenum Press, New York.

Fine, B.J. (1963). Introversion-Extraversion and Motor Vehicle Driver Behavior. Perceptual and Motor Skills, 12, 95-100.

Finn, P. \& Bragg, B. W. E. (1986). Perception of the risk of an accident by young and older drivers. Accident Analysis and Prevention, 18, 289-298.

Fry, G. A. (1955). Physiological Basis of Disability Glare. $13^{\text {th }}$ Session of the Commission International de l'Eclairage, Zurich.

Funder, D.C. (2001). The Personality Puzzle. W.W.Norton and Company, Inc., New York.

Gellatly, A.W. \& Weintraub, D.J. (1990). User Reconfigurations of the DeBoer Rating Scale for Discomfort Glare. Ann Arbor, Michigan.

Gilbert, D.G. \& Hagen, R.L. (1985). Electrodermal Response to Noise Stressors; Nicotine Extraversion Interaction. Personality and Individual Differences, 6, 573578.

Goldberg, L.R., (1981). Language and Individual Differences: The Search for Universals in Personality Lexicons". In L. Wheeler (Ed.), Review of Personality and Social Psychology, Beverly Hills, CA.

Goldberg, L. (1992). The Development of Markers for the Big-Five Factor Structure. Psychological Assessment, 4, 26-42.

Goldberg, L. (1999). The International Personality Item Pool Representation of the NEO PI-R [On-line]. Retreived September, 2002 from http://cac.psu.edu/ j5j/test/ipipneo1.htm

Goldberg, L.R. (2000). The International Personality Item Pool: A Scientific Collaboratory for the Development of Advanced Measures of Personality and Other Individual Differences [online]. Retrieved September, 2002 from http://ipip.ori.org/ipip/

Green, R.G. (1984). Preferred Stimulation Levels in Introverts and Extroverts: Effects on Arousal and Performance. Journal of Personality and Social Psychology, 46 (6), June 1984, 1303-1312, American Psychological Association.

Gresset, J.A. \& Meyer, F.M. (1994). Risk of Accidents among Elderly Car Drivers with Visual Acuity Equal to 6/12 or 6/15 and Lack of Binocular Vision. Ophthalmic and Physiological Optics, 14(1), 33-37.

Haegerstrom-Portnoy, G., Brabyn, J.A., Schneck, M.E., \& Jampolsky, A. (1997). The SKILL Card: An acuity test of reduced luminance and contrast. Smith-Kettlewell 
Institute Low Luminance, Investigative Ophthalmology and Visual Science, 38(1), 207-218.

Haslam, D.R., (1966). Individual Differences in Pain Threshold and the Concept of Arousal (Ph.D. Thesis).

Helson, R. \& Moane, G. (1987). Personality Change in Women from College to MidLife. Journal of Personality and Social Psychology, 53, 176-186.

Higgins, K.E., Wood, J.M., \& Tait, A. (1998). Vision and Driving: Selective Effect of Optical Blur on Different Driving Tasks. Human Factors, 40(2), 224-232.

Hills, B.L. (1980). Vision, Visibility and Perception in Driving. Perception, 9, 183-216. Holladay, L.L (1926). The fundamentals of glare and visibility. J Opt Soc Am, 12, 492.

Holladay, J.T., Prager, T.C., Trujillo, J., \& Ruiz, R.S. (1987). Brightness Acuity Test and Outdoor Visual Acuity in Cataract Patients. Journal of Cataract and Refractive Surgery, 13(1), 67-69.

Isen, A.M.M. \& Shalker, T.E. (1982). The Effect of Feeling State on Evaluation of Positive, Neutral, and Negative Stimuli: When you "Accentuate the Positive" do you "Eliminate the Negative"? Social Psychology Quarterly, 45, 58-63.

Ivers, R.Q., Mitchell, P., \& Cumming, R.G. (1999). Sensory Impairment and Driving: The Blue Mountains Eye Study. American Journal of Public Health, 89(1), 85-87.

Jang, Y., Mortimer, J.A., Haley, W.E., \& Borenstein Graves, A. (2002). The Role of Neuroticism in the Association Between Performance-Based and Self-Reported Measures of Mobility. Journal of Aging and Health, 14 (4), November 2002, 495508.

Jayle, G.E., Ourgaud, A.G., Baisinger, L.F., Holmes, W.J., \& Sir Duke-Elder, Stewart, (1959). Night Vision. Illinois

Johansson, K., Bronge, L., Lundberg, C., Persson, A., Seideman, M., \& Vitanen, M. (1996). Can a Physician Recognize and Older Driver with Increased Crash Risk Potential? Journal of the American Geriatrics Society, 44(10), 1198-1204.

John, O.P. (1990). The Big Five Factor Taxonomy: Dimensions of Personality in the Natural Language and in Questionnaires. In L.A. Pervin (Ed.), Handbook of Personality: Theory and Research, Guilford Press, 66-100, New York.

Jonah, B. A. (1986). Accident risk and risk-taking behavior among young drivers. Accident Analysis and Perception, 18, 255-271.

Judge, T.A. \& Ilies, R. (2002). Relationship of Personality to Performance Motivation: a Meta-Analytic Review. Apply Psychology, 87(4), 797-807.

Kline, D., Schieber, F., Abusamra, L.C., \& Coyne, A.C. (1983). Age, the Eye, and the Visual Channels: Contrast Sensitivity and Response Speed. Journal of Gerontology, 38, 211-216.

Kogan, N. \& Wallach, M. A. (1964). Risk Taking; A Study in Cognition and Personality. New York.

Larsen, R.J. \& Ketelaar, T. (1991). Personality and Susceptibility to Positive and Negative Emotional States. Journal of Personality and Social Psychology, 61, 132-140.

Lawrence A. Pervin \& Oliver P. John (2001). Personality: Theory and Research $8^{\text {th }}$ edition. University of California, Berkeley, John Wiley and Sons, Inc. 
Lennie, P. \& Van Hemel, S. B. (2002). Visual Impairments: Determining Eligibility for Social Security Benefits. National Research Council, National Academy Press, Washington, D.C.

Lockhart, T.E. (2002). Assessment of vision characteristics between younger and older adults. Virginia Polytechnic Institute and State University Interim Report 10-1502, Virginia.

Madden, D.J. \& Greene, H.A. (1987). From Retina to Response: Contrast Sensitivity and Memory Retrieval during Visual Word Recognition. Experimental Aging Research, 13, 15-21.

Mantyjarvi, M., Juntunen, V., \& Tuppurainen, K. (1998). Visual Functions of Drivers Involved in Traffic Accidents. Department of Opthalmology, University Hospital of Kuopio, POB 1777, 70211, Kuopio, Finland

Marottoli, R.A., Cooney, M., Wagner, D.R., Doucette, J., \& Tinetti, M.E. (1994). Predictors of Automobile Crashes and Moving Violations among Elderly Drivers. Analysis of Internal Medicine, 121(11), 842-846.

Martin, L., (1999). Computerized Method of Measure Glare and Contrast Sensitivity in Cataract Patients. Journal of Cataract and Refractive Surgery, 25(3), 411-415.

Mayer, R. E. \& Treat, J. R. (1977). Psychological, social and Cognitive Characteristics of High Risk Drivers. Accident Analysis and Prevention, 9, 1 - 8.

McBride, N.L. (2001). An Item Response Theory Analysis of the Scales from the International Personality Item Pool and the NEO Personality Inventory-Revised, Master Thesis. Virginia Polytechnic Institute and State University, Virginia.

McCloskey, L.W., Koepsell, T.D., Wolf, M.E., \& Buchner, D.M. (1994). Motor Vehicle Collision Injuries and Sensory Impairments of Older Drivers. Age and Ageing, 23(4), 267-273.

McCrae, R.R. \& Costa, P.T., Jr. (1991). Adding Liebe und Arbeit: The full five-factor model and well-being. Personality and Social Psychology Bulletin, 17, 227-232.

McCrae, R.R. \& Costa, P.T., Jr. (1994). The Stability of Personality: Observations and Evaluations. Current Directions in Psychological Science, 3, 173-175.

McCrae, R.R. \& Costa, P.T.Jr. (1997). Personality Trait Structure as a Human Universal. American Psychologist, 52, 509-516.

McEwen, W.K. (1959). The Yellow Pigment of Human Lenses. Amer. J. Ophthal., 47, 144-146.

McFarland, R.A. (1968). The Sensory and Perceptual Processes in Aging. In K.W. Schaie (Ed.). Theory and Methods of Research in Aging, Morgantown, WV.

McFarland, R.A., Domey, R.G., Warren, A.B., \& Ward, D.C. (1960). Dark Adaptation of a Function of Age: A Statistical Analysis. Journal of Gerontology, 15, 149-154.

McLaughlin, R.J. \& Harrison, N.W., (1974). Extraversion, Neuroticism and the Volunteer Ssubject. Psychological Reports, 32(3, Pt. 2), Jun 1973: 1131-1134.

Miller \& Benedek (1973). Intraocular Light Scattering. Illinois.

Miller, D., \& Nadler, D.J. (1990). Glare and Contrast Sensitivity for Clinicians. SpringerVerlag, New York.

Morris, L.W., (1967). Extraversion and Introversion: An Interactional Perspective. Hemisphere Publishing Corporation, New York. 
Murray, I. \& Plainis, S. (2001). Objective Assessment of Discomfort Glare. University of Manchester Institute of Science and Technology, U.K., retrieved October 2002 from http://www2.umist.ac.uk/optometry/dept/plainis/Discomfort_Glare.html.

National Research Council, (1994). Measurement of Visual Field and Visual Acuity for Disability Determination. National Academy Press, Washington, D.C.

Neter, J., Kutner, M.H., Nachtsheim, C.J., \& Wasserman, W. (1996). Applied Linear Statistical Models $3^{\text {nd }}$ Edition. Chicago, IL.

Neumann, A.C., McCarty, G.R., Locke, J., \& Cobb, B. (1988).Glare Disability Devices for Cataractous Eyes: A Consumer's Guide. Journal of Cataract and Refractive Surgery, 14(2), 212-216.

Norton, T.T., Corliss, D.A., \& Bailey, J.E. (2002). The Psychophysical Measurement of Visual Function. Butterworth Heinemann.

Olson, P.L \& Sivak, M (1984). Discomfort Glare from Automobile Headlights. Journal of the Illuminating Engineering Society, 13, 296-303.

Owsley, C., Ball, K.K., McGwin, G., Sloane, M.E., Roenker, D.L., Whiter, M.F., \& Overley, E.T. (1998). Visual Processing Impairments and Risk of Motor Vehicle Crash among Older Adults. The Journal of the American Medical Association, 279(14), 1083-1088.

Owsley, C., Ball, K.K., Slone, M.E., Roenker, D.L., \& Bruni, J.R. (1991). Visual/cognitive Correlates of Vehicle Accidents in Older Drivers. Psychology and Aging, 6(3), 403-415.

Owsley, C., McGwin, G., Sloane, M.E., Stalvey, B.T., \& Wells, J. (2001). Timed Instrumental Activities of Daily Living (TIADL) Tasks: Relationship t Visual Function in Older Adults. Optometry and Vision Science, 78(5), 350-359.

Pelli, D.B., Robson, J.G., \& Wilkins, A.J. (1988). The Design of a New Letter Chart for Measuring Contrast Sensitivity. Clinical Vision Sciences, 2(3), 187-199.

Pelz, D. C. \& Schuman, S. H. (1968). Dangerous Young Drivers. Society of Automotive Engineers Journal, 76, $61-68$.

Petherbridge, B. \& Hopkinson, R.G. (1950). Discomfort Glare and the Lighting on Building. Trans Illum Eng Soc (Lond), 15, 39.

Piedmont, R.L. \& Weinstein, H.P. (1994). Predicting Supervisor Ratings of Job Performance using the NEO Personality Inventory. Journal of Psychology, 128, 255-265.

Pitts, D. (1982). The Effects of Aging on Selected Visual Functions: Dark Adaptation, Visual Acuity, Stereopsis and Brightness Contrast. Aging in Human Visual Functions, New York.

Polyak, S.L. (1957). The Vertebrate Visual System. Chicago.

Pulling, N. H., Wolf, S. P., Sturgis, D. R., Vaillancourt, D. R., \& Dolliver, J. J., (1980). Headlight Glare Resistance and Driver Age. Human Factors, 22, 103-112.

Ranney, T.A., Masalonis, A.J., \& Simmons, L.A., (1996). Immediate and long-term effects of glare from following vehicles on target detection in a driving simulator. Transporation Research Record 1550. National Academy Press, Washington, DC.

Ranney, T. A., Simmons L.A., \& Masalonis, A. J. (1999). Prolonged exposure to glare and driving time: effects on performance in a driving simulator. Accident Analysis and Prevention, 31, 601-610. 
Ranney, T. A., Simmons L. A., \& Massalonis A. J. (2000). The immediate effects of glare and electrochromic glare-reducing mirrors in simulated truck driving. Human Factors, 42, 337-347.

Regan, D. (1991). Spatiotemporal Abnormalities of Vision in Patients with Multiple Sclerosis. Vision and Visual Dysfunction, Spatial Vision, MacMillan Press, London, 10, 239-247.

Roberts, B.W. \& Del Vecchio, W.F. (2000). The Rank-Order Consistency of Personality Traits from Childhood to Old Age: A Quantitative Review of Longitudinal Studies. Psychological Bulletin, 126, 3-25.

Rusting, C.L., (1998). Personality, Mood, and Cognitive Processing of Emotional Information: Three Conceptual Frameworks. Psychological Bulletin, 124 (2): 165-196.

Salgado, J.F. \& Rumbo, A. (1994). Personality and Job Performance in Financial Services Mangers. Unpublished manuscript, University of Santiago de Compostela

Sanders, M.S. \& McCormick, E.J. (1990). Human Factors in Engineering and Design $7^{\text {th }}$ Edition. McGraw Hill, New York.

Sanders, M., shaw, B., Nicholson, B. \& Merritt, J. (1990). Evaluation of Glare from Center-High-Mounted Stop Light. National Highway Traffic Safety Administration, Washington, DC.

Schieber, F., Kline, D.W., Kline, T.J.B., \& Fozard, J.L. (1992). The Relationship between Contrast Sensitivity and the Visual Problems in Older Drivers (SAE Technical Paper No.920613). Society of Automotive Engineers, Inc, Warrendale, PA.

Schiflett, S. G., Cadena, D. G., \& Hemion, R. H. (1969). Headlight Glare Effects on Driver Fatigue. Report No. AR-699. Final Report for Federal Highway Administration, U.S.D.O.T., Clearinghouse for Federal Scientific and Technical Information, Springfield, VA.

Schuerger, J.M., Zarrella, K.L., \& Hotz, A.S. (1989). Factors that Influence the Temporal Stability of Personality by Questionnaire. Journal o Personality and Social Psychology, 56, 1218-1223.

Schuman, S. H., Pelz, D. C., Ehrlich, N. J., \& Selzer, M. L. (1967). Young male Drivers: Impulse Expression, Accidents, and Violations. Journal of the American Medical Association, 200, 1026 - 1030.

Shaw, L. \& Sichel, H. (1970). Accidnet Proneness. Pergamon Press, Oxford.

Shinar, D. \& Schieber, F. (1991). Visual Requirements for Safety and Mobility of Older Drivers. Human Factors, 33, 507-519.

Singh, A.P. (1978). Neuroticism, Extraversion, and Accidents. India Psychological Review, 16, 41-45.

Sivak, M. \& Olson, P.L. (1982). Nighttime Legibility of Traffic Signs: Conditions Eliminating the Effects of Driver Age and Disability Glare. Accident Analysis and Prevention, 14(2), 87-93.

Sivak, M. \& Olson, P. (1987). Toward the Development of a Field Methodology for Evaluating Discomfort Glare from Automobile Headlamps (Rept. No. UMTRI87-41). Ann Arbor, Michigan.

Sivak, M., Simmons, C.J., \& Flannagan, M. (1990). Effect of Headlamp Area on Discomfort Glare. Lighting Research and Technology, 22, 49-52. 
Sivak, M., Flannagan, M., Ensing, M., \& Simmons, C.J. (1991). Discomfort Glare is Task Dependent. International Journal of Vehicle Design, 12(2), 152-159.

Sivak, M. \& Flannagan, M.J. (1994). Recent Steps toward International Harmonization of the Low-beam Headlighting Patterns. International Journal of Vehicle Design, 15(3-5), 223-233.

Slater, E. \& Slater, P. (1944). A Heuristic theory of Neurosis. 7, 49-55.

Smith, S.L., (1968). Extraversion and Sensory Threshold. Psychophysiology, 5(3), 293299. Cambridge University Press.

Snell, A.C. (1925). Visual Efficiency of Various Degrees of Subnormal Visual Acuity: It's Effect on Earning Ability. The Journal of the American Medical Association, 85(18), 1367-1373.

Stanley Coren, Clare Porac, \& Lawlence M. Ward (1979). Sensation and Perception. Academic Press, New York.

Stelmack, R. M. (1990). Biological Bases of Extraversion: Psychophysiological Evidence. Journal of Personality, March, 58(1), 293-311.

Stelmack, R. M., Achorn, E., \& Michaud, A. (1977). Extraversion and Individual Differences in Auditory evoked response). Psychophysiology, 14, 368-374.

Theeuwes, J. \& Alferdinck, W.A.M. (1996). The Relation Between Discomfort Glare and Driving Behavior. Report DOT HS 808 452, National Highway Traffic Safety Administration, Washington DC.

Theeuwes, J. \& Alferdinck W.A.M., Michael, P. (2002). Relation Between Glare and Driving Performance. Human Factors, 44(1), 95-107.

Trimpop, R. \& Kirkcaldy, B (1996). Personality Predictors of Driving Accidents. Work and Organisational Psychology Unit, University of Jena, Jena, Germany.

Troncoso, M.U. (1942). The Intrascleral Vascular Plexus and its Relations to the Aqueous Outflow. Am. J. Ophthalmol., 25, 1153-1162.

Vos, J. J. (1984). Disability Glare: A State-of-the-art report. J of the CIE, 2, 39-53.

Wagman, I. \& L.M. Nathanson (1942). Influence of Intensity of White Light upon Pupil Diameter of the Human and of the Rabbit. Proc. Soc. Exp. Biol. Med., 49, 466470.

Watson, D. \& Clark, L.A. (1984). Negative Affectivity: The Disposition t Experience Aversive Emotional States. Psychological Bulletin, 96, 465-490.

Watson, D. \& Pennebaker, J. (1989). Health Complaints, Stress, and Distress: Exploring the Central Role of Negative Affectivity. Psychological Review, 96, 234-254.

Watson, D. \& Clark, L.A. (1992). On Traits and Temperament: General and Specific Factors of Emotional Experience and their Relation to the Five-Factor Model. Journal of Personality, 60, 441-476.

Weale, R. A. (1963). The Aging Eye. Harper and Row, London.

Wierwille, W. W., Lee, S. E., \& DeHart, M. C. (2003). Testing and Optimization of High-Level and Stopped/Slowly-Moving Vehicle Rear-Signaling Systems. Task 2 Report for National Highway Traffic Safety Administration contract DTNH 2299-C-07235 (Report DOT HS 809 597). U.S. Department of Transportation, Washington, DC.

Weisen, A. (1965). Differential Reinforcing Effects of Onset and Offset of Stimulation on the Operant Behavior of Normals, Neurotics, and Psychopaths, Unpublished $\mathrm{Ph}$.D. thesis. University of Florida. 
Weymough, F.W. et al. (1928). Visual Acuity within the Area Centralis and its Relation to Eye Movements and Fixation. Am. J. Ophthalmol, 11, 947.

Wigglesworth, M.J. \& Smith, B.D. (1976). Habituation and Dishabituation of the Electrodermal Orienting Reflex in Selection to Extraversional Neuroticism. Journal of Research in Personality, 10, 437-445.

Wilson, R. \& Greensmith, J. (1983). Multivariate Analysis of the Relationship between Drivometer Variables and Drivers' Accident, Sex and Exposure Status. Human Factors, 25, 303-312.

Wolf, E. W. (1960). Glare and Age. Archives of Ophthalmology, 64, 502-514.

Wood, J.M. \& Troutbeck, R. (1992). Effect of Restriction of the Binocular Visual Field on Driving Performance. Ophthalmic and Physiological Optics, 12(3), 291-298.

Wood, J.M., Dique, T., \& Troutbeck, R. (1993). The Effect of Artificial Visual Impairment on Functional Visual Fields and Driving Performance. Clinical Vision Sciences, 8, 563-575. 


\section{APPENDIX A: SCREENING TEST RESULTS}

\section{A1. Personality Test}

\section{Neuroticism Score:}

Taking into account all 78 responses on 20 neuroticism trait questions, the frequency plots of 5 interval data range is shown below (Figure 36).

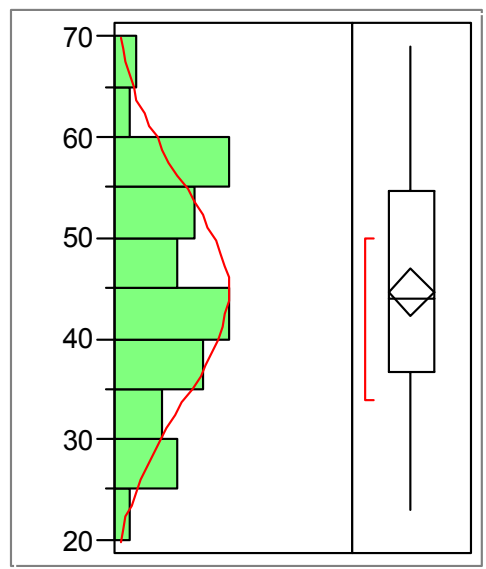

Figure 36: The Frequency Distribution of Neuroticism Scores

The maximum score on neuroticism trait was 69 while the minimum score on this trait was 23 . The mean value was 44.62 and the standard deviation was 10.97 . Using the $1 / 3$ cut off technique, the high and low scorer group was determined by having neuroticism trait score higher than 50 and lower than 38 respectively. Note that the difference between two groups was at 12 .

\section{Extraversion Score:}

Similarly, taking into account all 78 responses on 20 extraversion trait questions, the frequency plots of 5 interval data range was shown in Figure 37. The maximum score on extraversion trait was 96 while the minimum score on this trait was 44 . The mean value was 70.67 and the standard deviation was 13.136 . Using the $1 / 3$ cut off technique, 
the high and low score groups was determined by having extraversion score higher than 79 and lower than 66 respectively. Note that difference between two groups was 13.

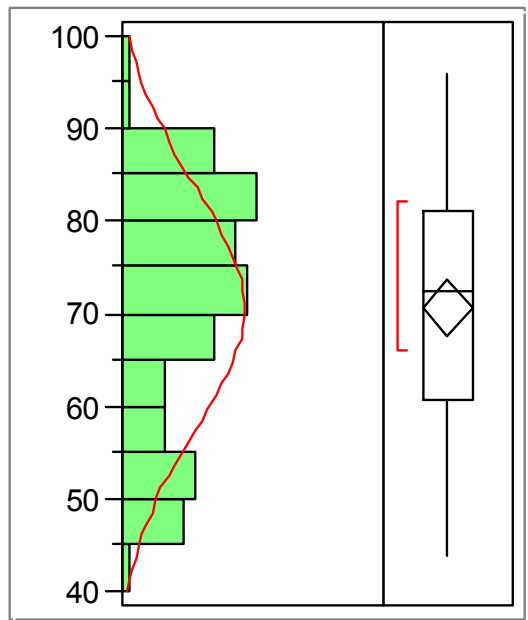

Figure 37: The Frequency Distribution of Extraversion Scores

Determined Trait Group for the Extreme Score Participants:

Using the previously mentioned technique, the participant pool for this study became as shown in the following table (Table 38 ).

Table 38: Participants Pool for Extreme Score on either Neuroticism or Extraversion Trait

\begin{tabular}{cccccc}
\hline Trait & \multicolumn{2}{c}{ Male } & \multicolumn{2}{c}{ Female } & \multirow{2}{*}{ Total } \\
Group & Elderly & Young & Elderly & Young & \\
\hline HN & 1 & 3 & 3 & 10 & 17 \\
LN & 3 & 8 & 4 & 3 & 18 \\
HE & 2 & 3 & 3 & 11 & 19 \\
LE & 3 & 6 & 3 & 1 & 13 \\
& $\mathbf{9}$ & $\mathbf{2 0}$ & $\mathbf{1 3}$ & $\mathbf{2 5}$ & $\mathbf{6 7}$ \\
\hline
\end{tabular}

Also, because four participants were categorized into more than one personality groups, they were demonstrated in the following separate table ( Table 39). The two HN, LE participants were later assigned as LE group and the LN, HE participant were tested as $\mathrm{LN}$ group. 
Table 39: Participants Pool for Extreme Score on Both Neuroticism and Extraversion Trait

\begin{tabular}{cccccc}
\hline Trait & \multicolumn{2}{c}{ Male } & \multicolumn{2}{c}{ Female } & \multirow{2}{*}{ Total } \\
Group & Elderly & Young & Elderly & Young & \\
\hline LN, LE & 1 & & & & 1 \\
LN, HE & & & 1 & & 1 \\
HN, LE & & 2 & & & 2 \\
HN, HE & & & & 1 & 1 \\
& $\mathbf{1}$ & $\mathbf{2}$ & $\mathbf{1}$ & $\mathbf{1}$ & $\mathbf{5}$ \\
\hline
\end{tabular}

\section{A2. Vision Tests}

After categorizing group, all participants were contacted to come to the glare experiment and tested on two vision tests for screening purpose. The tests included the contrast sensitivity (CS) and nighttime visual acuity tests. Because some of the participants had already tested on these tests during fall, 2002, those participants who passed the vision tests got priority to come to this study. However, as some participants cannot volunteer to come back, the other twelve new participants were required to come for these two screening tests. Among these twelve participants, six were young female and another six were young male. Nonetheless, one of these twelve cannot pass the CS screening test. Including all available participants from previous study and the new eleven participants who passed all screening tests, the demographic data of these thirty six individuals are demonstrated in the following table (Table 40).

Table 40: Demographic Data of Participants included in the Study

\begin{tabular}{cccccc}
\hline Trait & \multicolumn{2}{c}{ Male } & \multicolumn{2}{c}{ Female } & \multirow{2}{*}{ Total } \\
Group & Elderly & Young & Elderly & Young & \\
\hline HN & 0 & 2 & 1 & 6 & 9 \\
LN & 1 & 5 & 2 & 1 & 9 \\
HE & 2 & 2 & 0 & 5 & 9 \\
LE & 1 & 5 & 2 & 1 & 9 \\
& $\mathbf{4}$ & $\mathbf{1 4}$ & $\mathbf{5}$ & $\mathbf{1 3}$ & $\mathbf{3 6}$ \\
\hline
\end{tabular}


The vision screening results of these trait groups are shown in the following tables (Table 41). Note that the student t-test statistics used to compare screening performances between high and low score trait groups suggested insignificant difference performances between high and low neuroticism scorers as well as high and low extraversion scorers.

Table 41: Descriptive T-Test Summary Table for Vision Screening Results

\begin{tabular}{llll}
\hline Variable & High Scorer & Low Scorer & P-value \\
\hline Screening VA - Neuroticism Data & 0.8622 & 0.8600 & Not significant \\
& $(0.142634)$ & $(0.133791)$ & \\
Screening CS - Neuroticism Data & 5.02222 & 5.55556 & Not significant \\
& $(0.692419)$ & $(0.596052)$ & \\
Screening VA - Extraversion Data & 0.8222 & 0.7800 & Not Significant \\
& $(0.125477)$ & $(0.201742)$ & \\
Screening CS - Extraversion Data & 5.51111 & 5.34444 & Not Significant \\
& $(0.667915)$ & $(0.915302)$ & \\
\hline
\end{tabular}




\section{APPENDIX B: SECONDARY RESULTS AND DISCUSSION}

\section{B1. Relationship between Screening and Actual Performance}

The bivariate analysis results are demonstrated in the Table 42 below. In general, the result indicated low relationship except the one with CS as actual performance on extraversion data.

Table 42: Bivariate Regression Analysis between Screening and Actual Performance

\begin{tabular}{lll}
\hline Variable & $\mathrm{R}^{2}$ & $\mathrm{P}$-value \\
\hline Screening CS and CSI - N Data & 0.04968 & 0.3740 \\
Screening CS and CS - N Data & 0.10243 & 0.1057 \\
Screening VA and CSI - N Data & 0.01548 & 0.6228 \\
Screening VA and CS - N Data & 0.09535 & 0.1142 \\
Screening CS and CSI - E Data & 0.04061 & 0.2082 \\
$*$ Screening CS and CS - E Data & 0.67829 & $<.0001$ \\
Screening VA and CSI - E Data & 0.12109 & 0.0862 \\
$*$ Screening VA and CS - E Data & 0.37621 & 0.0040 \\
\hline
\end{tabular}

\section{B2. Age Factor}

Because the number participants in young and elderly groups in this study are highly unbalanced (9 elderly and 27 young), the secondary results on age factor will be analyzed using the Student's t-test (means comparison) for parametric data and the Median test for non-parametric data. 
Table 43: Descriptive ANOVA and T-Test Summary Table for Age Factor

\begin{tabular}{llll}
\hline Variable & Young & Elderly & P-value \\
\hline * Screening VA & 0.884444 & 0.671111 & Significant \\
& $(0.115603)$ & $(0.133832)$ & \\
* Screening CS & 5.52593 & 4.85556 & Significant \\
& $(0.675160)$ & $(0.674743)$ & \\
Contrast Sensitivity Index (CSI) & 2.6289759 & 2.8055404 & Not \\
& $(1.32894)$ & $(1.62479)$ & Significant \\
* Contrast Sensitivity (CS) & 4.02390 & 2.61889 & Significant \\
& $(1.29794)$ & $(0.97663)$ & \\
Subjective Rating & 5.15432 & 5.16667 & Not \\
& $(2.31927)$ & $(1.80278)$ & Significant \\
Illuminance (lux) & 61.6872 & 62.5526 & Not \\
& $(8.02228)$ & $(6.73644)$ & Significant \\
\hline
\end{tabular}

In general, the elderly group had significantly poorer performance in screening tests and also had lower CS under glare condition. The more detail for significant difference results were shown as follows:

$\underline{\text { Screening VA }}$

Table 44: Descriptive Summary of Rating on Gender Main Effect

\begin{tabular}{lrr}
\hline Age & Screening VA & Std. Dev. \\
\hline Young & 0.884444 & 0.115603 \\
Elderly & 0.671111 & 0.133832 \\
\hline
\end{tabular}

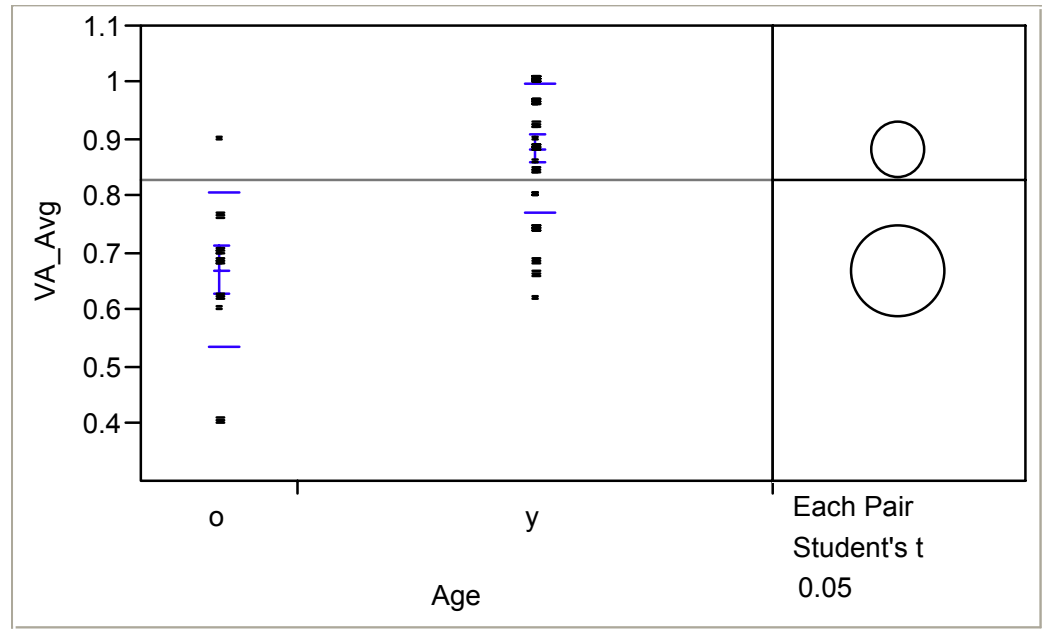

Figure 38: Age Main Effect on Visual Acuity 


\section{$\underline{\text { Screening CS }}$}

Table 45: Descriptive Summary of Screening CS on Gender Main Effect

\begin{tabular}{lrr}
\hline Age & Screening CS & Std. Dev. \\
\hline Young & 5.52593 & 0.675160 \\
Elderly & 4.85556 & 0.674743 \\
\hline
\end{tabular}

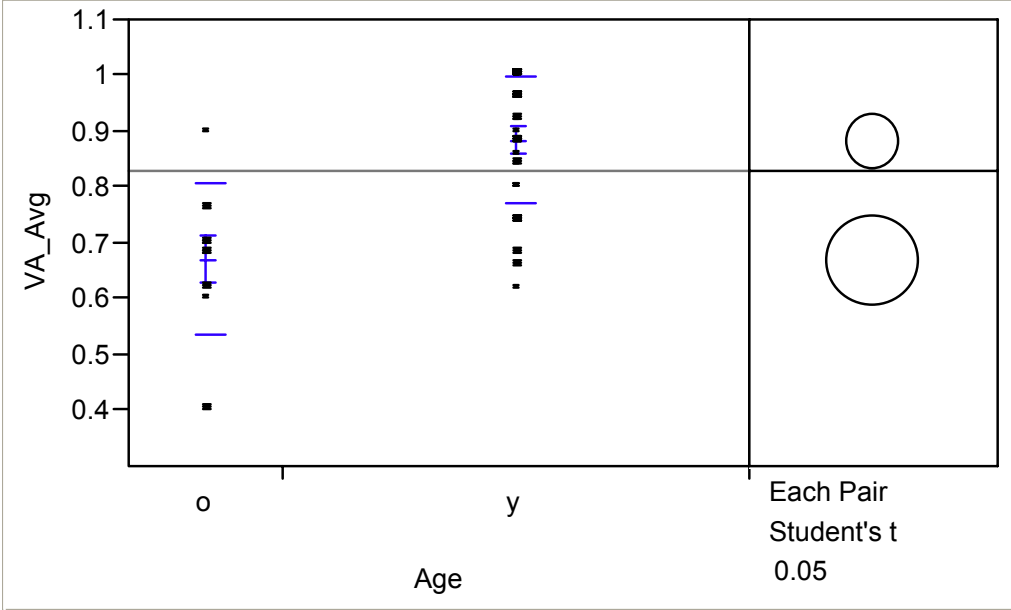

Figure 39: Age Main Effect on Screening CS

\section{Contrast Sensitivity (CS)}

Table 46: Descriptive Summary of CS on Age Main Effect

\begin{tabular}{lrr}
\hline Age & CS & Std. Dev. \\
\hline Young & 4.02390 & 1.29794 \\
Elderly & 2.61889 & 0.97663 \\
\hline
\end{tabular}

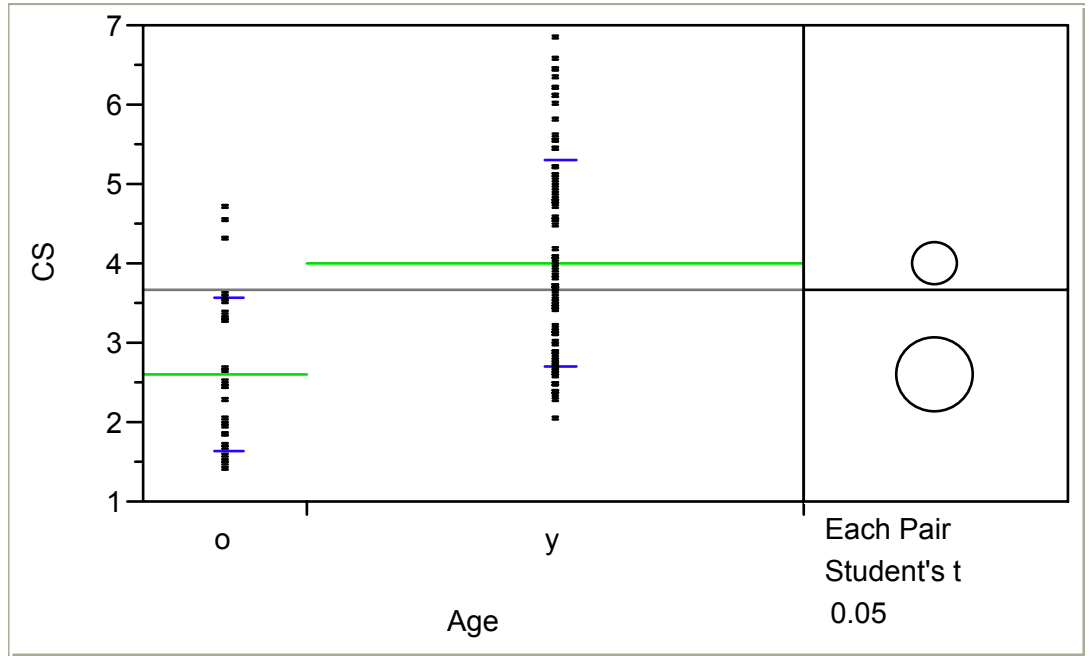


Note that although the CS was significantly difference between young and elderly group, the CSI performances of the two groups were not significantly difference. Last but not least, the subjective rating was found to be insignificant between age groups.

\section{B3. Gender Factor}

Because the number of male and female in this study were equal (18 male and 18 female) and each participant were tested with the same three glare levels, the 2-way ANOVA statistics (gender x glare level) was used to investigate the gender effect on all dependent variables. Note that the personality factor was not included into this model due to highly unbalanced of gender difference in the personality group. This unbalanced was especially found in neuroticism groups (high neuroticism group has 2 male and 7 female; low neuroticism group has 6 male and 3 female).

Table 47: Descriptive ANOVA Summary Table for Gender Factor

\begin{tabular}{llll}
\hline Variable & Male & Female & P-value \\
\hline * Contrast Sensitivity Index (CSI) & 2.45689 & 2.88934 & 0.0198 \\
& $(1.38118)$ & $(1.40351)$ & \\
Contrast Sensitivity (CS) & 3.69176 & 3.65354 & 0.8291 \\
& $(1.33666)$ & $(1.40627)$ & \\
* Subjective Response & 5.62963 & 4.68519 & 0.0081 \\
& $(1.95029)$ & $(2.33573)$ & \\
Illuminance (lux) & 61.1090 & 62.6980 & 0.4596 \\
& $(52.7533)$ & $(53.8564)$ & \\
\hline
\end{tabular}

The result indicated statistically significant differences CSI $\left(F_{1,102}=5.6029, p=\right.$ 0.0198) between two genders. In general, female had higher CSI (Table 48, Figure 41).

Table 48: Descriptive Summary of CSI on Gender Main Effect

\begin{tabular}{lrr}
\hline Gender & CSI & Std. Dev. \\
\hline $\mathrm{f}$ & 2.88934 & 1.40351 \\
$\mathrm{~m}$ & 2.45689 & 1.38118 \\
\hline
\end{tabular}




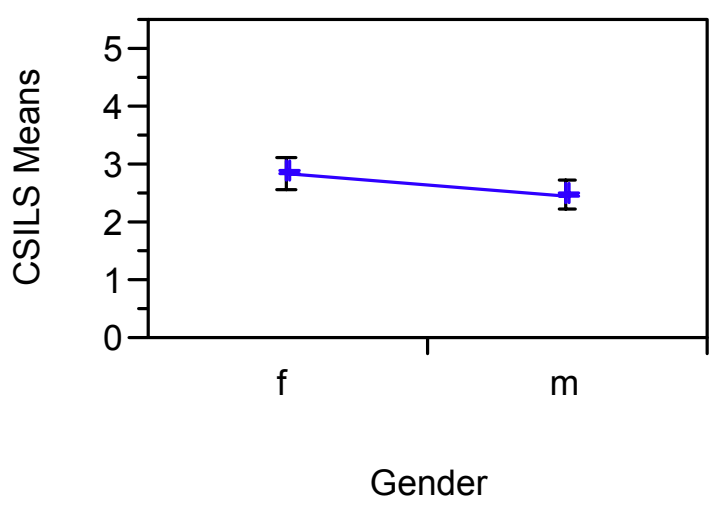

Figure 41: Gender Main Effect on CSI

Although significant, personality trait and/or age factors may have influenced this result. Similar to previous studies (e.g., Furnham \& Saipe, 1993), in this study, high neurotic group was composed of more numbers of female participants (i.e., 7 females in high $\mathrm{N}$ ). As a result, a female or high scorer on neuroticism participant could over-react to the glare situation, be more distractible by glare, and be less motivated to perform well.

On the other hand, the result indicated no statistically significant difference on CS $\left(F_{1,102}=0.0468, p=0.8291\right)$ between two genders. As indicated earlier, CS is strongly related to age. As such, similar numbers of elderly between genders (i.e., 4 elderly male and 5 elderly female) may have led to this insignificant result. Furthermore, the result indicated statistically significant differences $\left(F_{1,102}=7.2893, p=0.0081\right)$ between two genders. Female group gave lower rating than the male group (Table 49, Figure 42).

Table 49: Descriptive Summary of Rating on Gender Main Effect

\begin{tabular}{lrr}
\hline Gender & Rating & Std. Dev. \\
\hline $\mathrm{F}$ & 4.68519 & 2.33573 \\
$\mathrm{M}$ & 5.62963 & 1.95029 \\
\hline
\end{tabular}




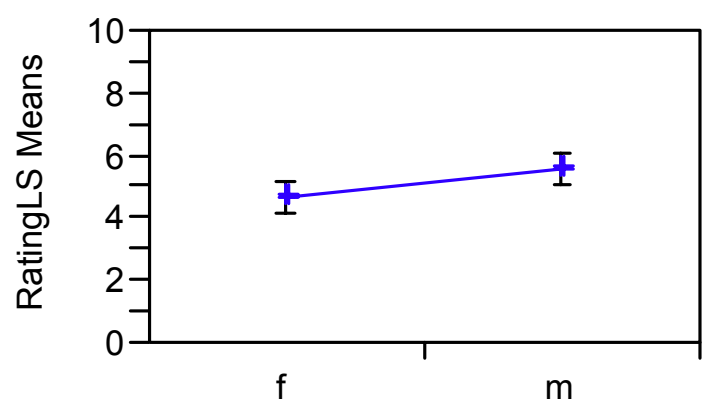

Gender

Figure 42: Gender Main Effect on Rating

\section{B4. Parsing Model (Partial out the Age and Personality Factors)}

To further investigate relationships among all dependent variables (i.e., contrast sensitivity index, contrast sensitivity, and rating response), the parsing method was used. Parsing method is referred to the data grouping (e.g., group of the elderly data). The Table 50 illustrated results from bivariate regression analysis including explained variance, p-value, and the direction of the linear relationship.

Table 50: Bivariate Analysis Using Parsing Data

\begin{tabular}{|l|c|c|c|c|c|c|}
\hline \multirow{2}{*}{$\begin{array}{c}\text { Used } \\
\text { Data }\end{array}$} & \multicolumn{2}{|c|}{ Contrast Sensitivity Index (CSI) } & \multicolumn{3}{c|}{ Contrast Sensitivity (CS) } \\
\cline { 2 - 7 } & Y + O & Young (Y) & Old (O) & Y +O & Young (Y) & Old (O) \\
\hline High N & $13.59 \%(+)$ & $10.75 \%(+)$ & - & $13.84(-)$ & $11.05 \%(-)$ & - \\
& 0.059 & 0.1177 & $(1$ participant) & 0.056 & 0.1125 & $(1$ participant) \\
\hline Low N & $3.19 \%(+)$ & $13.43 \%(+)$ & $0.11 \%(-)$ & $16.94 \%(-)$ & $53.49 \%(-)$ & $53.92 \%(-)$ \\
& 0.3727 & 0.1348 & 0.9334 & 0.033 & 0.0006 & 0.0243 \\
\hline All N & $8.59 \%(+)$ & $11.0 \%(+)$ & $4.60 \%(+)$ & $13.15 \%(-)$ & $18.70 \%(-)$ & $25.57 \%(-)$ \\
& 0.0315 & 0.0318 & 0.5032 & 0.007 & 0.0042 & 0.0935 \\
\hline High E & $28.77 \%(+)$ & $24 . .34 \%(+)$ & $14.78 \%(+)$ & $42.19 \%(-)$ & $38.50 \%(-)$ & $31.67 \%(-)$ \\
& 0.0039 & 0.023 & 0.4517 & 0.0002 & 0.0027 & 0.2449 \\
\hline Low E & $51.18 \%(+)$ & $37.81 \%(+)$ & $87.17 \%(+)$ & $7.78 \%(-)$ & $28.36 \%(-)$ & $2.28 \%(-)$ \\
& $<.0001$ & 0.0066 & 0.0002 & 0.1588 & 0.0229 & 0.6980 \\
\hline All E & $23.57 \%(+)$ & $26.33 \%(+)$ & $21.62 \%(+)$ & $16.08 \%(-)$ & $17.30 \%(-)$ & $13.35 \%(-)$ \\
& 0.0002 & 0.0008 & 0.0807 & 0.0027 & 0.0085 & 0.1804 \\
\hline N + E & $15.47 \%(+)$ & $17.26 \%(+)$ & $12.57 \%(+)$ & $14.32 \%(-)$ & $17.77 \%(-)$ & $17.98 \%(-)$ \\
& $<.0001$ & 0.0001 & 0.0695 & $<.0001$ & $<.0001$ & 0.0275 \\
\hline
\end{tabular}




\section{APPENDIX C: PROJECT QUESTIONNAIRE}

\section{Instruction}

On the following pages, there are phrases describing people's behaviors. Please use the rating scale below to describe how accurately each statement describes. Describe yourself as you generally are now, not as you wish to be in the future. Describe yourself as you honestly see yourself, in relation to other people you know of the same sex as you are, and roughly your same age. So that you can describe yourself in an honest manner, your responses will be kept in absolute confidence. Please read each statement carefully, and then mark $(\checkmark)$ in the box that corresponds to your reply

\begin{tabular}{|c|c|c|c|c|c|}
\hline List of Behaviors & $\begin{array}{c}\text { Very } \\
\text { Inaccurate }\end{array}$ & $\begin{array}{l}\text { Moderately } \\
\text { Inaccurate }\end{array}$ & $\begin{array}{c}\text { Neither } \\
\text { Inaccurate } \\
\text { nor Accurate }\end{array}$ & $\begin{array}{c}\text { Moderately } \\
\text { Accurate }\end{array}$ & $\begin{array}{c}\text { Very } \\
\text { Accurate }\end{array}$ \\
\hline \multicolumn{6}{|l|}{ Feel threatened easily } \\
\hline \multicolumn{6}{|l|}{ Keep others at a distance } \\
\hline \multicolumn{6}{|l|}{ Start conversations } \\
\hline \multicolumn{6}{|l|}{ Cheer people up } \\
\hline \multicolumn{6}{|l|}{ Fear for the worst } \\
\hline \multicolumn{6}{|l|}{ Am often down in the dumps } \\
\hline \multicolumn{6}{|l|}{ Have little to say } \\
\hline \multicolumn{6}{|l|}{ Am skilled in handling social situations } \\
\hline \multicolumn{6}{|l|}{ Don't mind being the center of attention } \\
\hline
\end{tabular}




\begin{tabular}{|c|c|c|c|c|c|}
\hline List of Behaviors & $\begin{array}{c}\text { Very } \\
\text { Inaccurate }\end{array}$ & $\begin{array}{l}\text { Moderately } \\
\text { Inaccurate }\end{array}$ & $\begin{array}{c}\text { Neither } \\
\text { Inaccurate } \\
\text { nor Accurate }\end{array}$ & $\begin{array}{c}\text { Moderately } \\
\text { Accurate }\end{array}$ & $\begin{array}{c}\text { Very } \\
\text { Accurate }\end{array}$ \\
\hline \multicolumn{6}{|l|}{ Rarely lose my composure } \\
\hline \multicolumn{6}{|l|}{ Am relaxed most of the time } \\
\hline \multicolumn{6}{|l|}{ Worry about things } \\
\hline \multicolumn{6}{|l|}{ Am not easily bothered by things } \\
\hline \multicolumn{6}{|l|}{ Seldom feel blue } \\
\hline \multicolumn{6}{|l|}{ Am not easily frustrated } \\
\hline \multicolumn{6}{|l|}{ Talk to a lot of different people at parties } \\
\hline \multicolumn{6}{|l|}{ Seldom get mad } \\
\hline \multicolumn{6}{|l|}{ Rarely get irritated } \\
\hline \multicolumn{6}{|l|}{ Avoid contacts with others } \\
\hline \multicolumn{6}{|l|}{ Find it difficult to approach others } \\
\hline \multicolumn{6}{|l|}{ Have frequent mood swings } \\
\hline \multicolumn{6}{|l|}{ Am the life of the party } \\
\hline \multicolumn{6}{|l|}{ Am hard to get to know } \\
\hline \multicolumn{6}{|l|}{ Retreat from others } \\
\hline \multicolumn{6}{|l|}{ Dislike myself } \\
\hline \multicolumn{6}{|l|}{ Am very pleased with myself } \\
\hline Would describe my experience as somewhat dull & & & & & \\
\hline
\end{tabular}




\section{APPENDIX D: LANDOLT'S C-CIRCLE CHART}

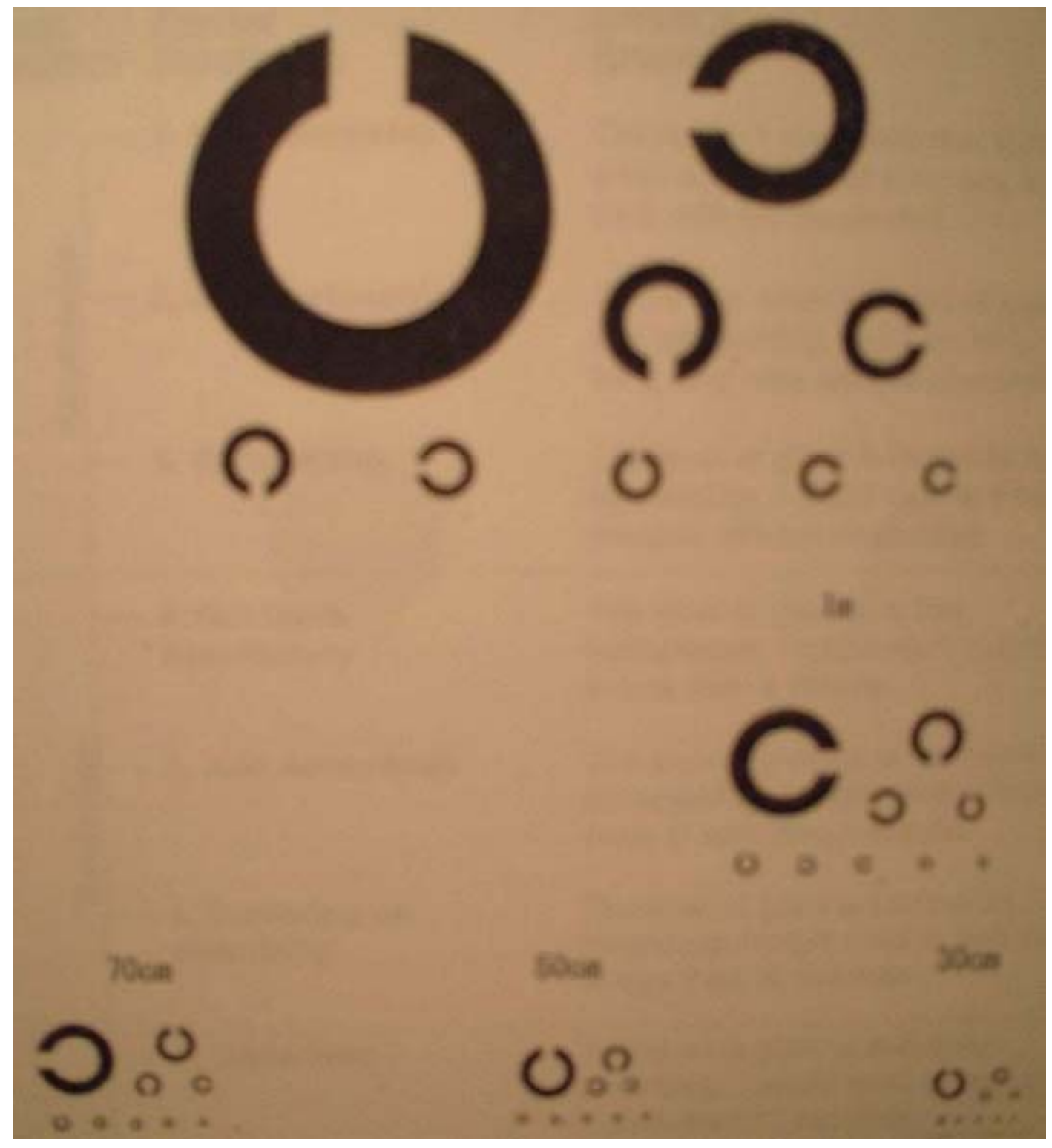




\section{APPENDIX E: INSTRUCTION EXAMPLE}

Visual Acuity Test: Asked participants to report the smallest Landolt circle that s/he can still detect direction of the broken gap.

"On the screen, you will see a set of circles with a broken gap in each of them. Please report the smallest circle which you can still be able to detect the direction of the gap on the outer edge of the circle and also report the orientation of that gap whether it's up, bottom, left, or right."

Vistech Contrast Sensitivity Test: Asked participants to determine the slanted direction of bars for all patches. The instruction was extracted from Vistech Chart Manual.

"Your task is to read across each row, starting with row A, patch 1, and call out whether the patch is oriented to the left, right, straight up and down or patches are very low in contrast and you may not see any bars in theses patches. If this is the case, simply answer "blank". However, if you do see something in a patch but your are not sure of the orientation, you are allowed to guess"

Contrast Sensitivity Test: Asked participants to perform the contrast threshold test under glare condition.

1. Ascending Trial: "On the screen, there will be a broken circle similar to the letter $\mathrm{C}$ in the middle of it. I will continually increase the image's contrast so that it becomes more detectable. At each increasing level, I will ask you to report whether you could detect the C target or not. If "yes" response is received, I will ask you to report the direction of the broken gap. The two successive correct answers of the gap direction will stop the trial. However, if "no" responses are received or gap direction responses are incorrect, I will continually increase contrast level of the target to next successive levels"

2. Descending Trial: "In contrary to last trial, I will continually decrease the image's contrast so that it becomes less detectable. At each decreasing level, I will ask you to report whether you could still detect the $\mathrm{C}$ target or not. If yes, I will continually decrease 
contrast of the target to next successive levels. Once you report as no, I will increase the contrast level to previous step and ask for the direction of opening similar to the previous trial. The two successive correct answers of the gap direction will stop the trial. However, if responses are incorrect, I will continually increase contrast level of the target to next successive levels"

Subjective Rating: Asked participant to verbally give the subjective glare rating. "Please indicate on the discomfort scale from one to nine what you thought about the light source you are exposed to?" 


\section{APPENDIX F: DISCOMFORT GLARE RATING SCALE}

Discomfort glare is glare that a person finds uncomfortable to a greater or lesser degree. Please rate your level of discomfort glare for the light setting by placing a slash mark on the following scale at the point that most closely matches your perception of the discomfort glare level (note that response can be placed between numbers).

\section{General Precise \\ $\underline{\text { Description }} \underline{\text { Description }} \underline{\underline{\text { Reaction }}}$}

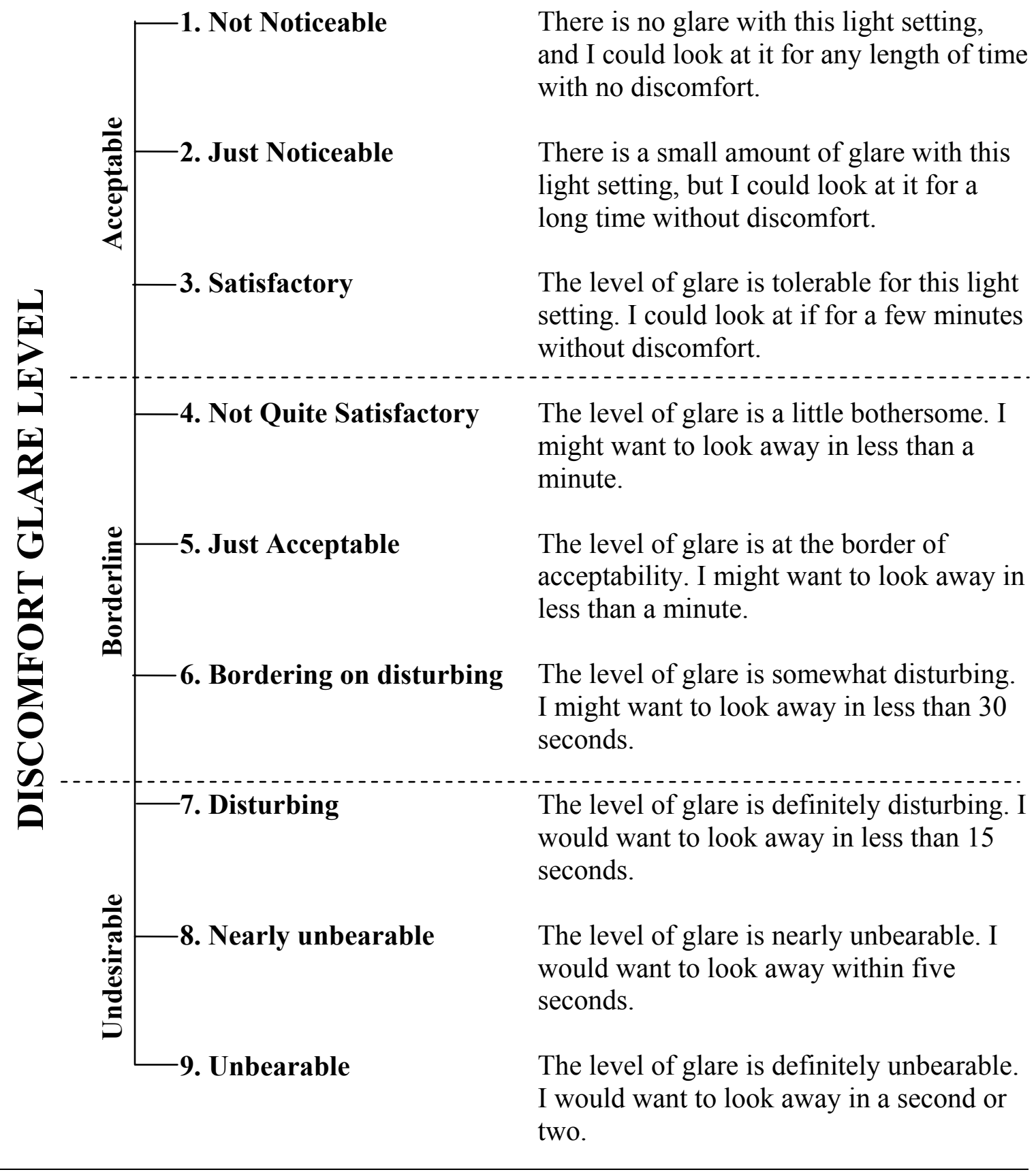




\title{
APPENDIX G: INFORMED CONSENT FORM
}

\author{
Virginia Polytechnic Institute and State University \\ Informed Consent for Participants of Investigative Projects \\ Grado Department of Industrial and Systems Engineering \\ Virginia Tech
}

TITLE: Trait-Based Individual Differences on Discomfort Glare Rating Responses and Related Visual Contrast Sensitivity

\section{PURPOSE OF PROJECT}

The purpose of the project is to provide a better understanding of glare discomfort experience occurred in nighttime driving condition and related vision performance (contrast sensitivity).

\section{PROCEDURE}

1. Read and sign this Informed Consent Form.

2. Participate in familiarize session

3. Participate in glare study session

\section{$\underline{\text { Familiarize Session }}$}

First, you will be given with a glare discomfort scale (1 - Not noticeable, to 9 Unbearable). Experimenter will describe the meaning of each of the terms used in the scale. You will be required to practice on reporting the subjective responses. After that, you will be asked to sit on a chair in front of a laptop screen using as the viewing target under nighttime condition. Nighttime condition will be provided by dimming the lighting levels in a blacken room. A contrast threshold test will be conducted under no glare condition to ensure the understanding of detectable target level.

\section{Glare Study Session}

For the glare test, illumination from a constant glare source will be differently directed towards your eyes for several times. You will be asked to rate comfort levels of glare illuminations utilizing the discomfort glare rating scale for all trials. Additionally, a few repeated contrast threshold tests will be conducted for each glare manipulated levels. The total of three manipulated glare levels will be used in defined order.

For all glare trials, you will be allowed to rest your eyes for at least 1 minute after expose to each glare level. During the resting period, you might want to close your eyes if needed.

\section{RISKS OF PARTICIPATION}

There are no major physical or emotional risks associated with this research. Only minor irritation of the eyes and momentary blur vision are expected to occur. The 
mentioned risks will be minimized through 1 minute resting of the eyes and minimizing exterior reflected glare via painted wall.

\section{BENEFITS}

While there are no direct benefits to you from this research, you may find the experiment interesting and gain the appreciation of contributing to the research findings. No promise or guarantee of benefits is made to encourage you to participate. Your participation may provide a better understanding of your visual perception characteristics during nighttime glare conditions. In addition, you can receive the results of this research directly from the experimenter after the data have been analyzed. Contact information is provided at the end of this form.

\section{COMPENSATION}

Monetary compensation will be provided ( $\$ 10.00$ per hour) when the entire research session has been completed.

\section{ANONYMITY AND CONFIDENTIALITY}

The data from this study will be kept strictly confidential. No data will be released to anyone but the principal investigators without written consent of the subject. Data will be specifically identified using participant's ID number.

\section{FREEDOM TO WITHDRAW}

You are free to withdraw at any time from the study for any reason without penalty. If you withdraw before completing the session, you will be compensated for the portion of the time you have spent participating in the study. With very low probability, circumstances may come up that the researcher will determine that you should not continue as a participant in the study. For example, an illness could be a reason to have the researcher stops your participation in the study.

\section{APPROVAL OF RESEARCH}

This research has been approved, as required, by the Institutional Review Board for Research Involving Human Subjects at Virginia Tech, and by the Grado Department of Industrial and Systems Engineering. You could receive a copy of this form to take with you if you wish.

\section{PARTICIPANT'S RESPONSIBILITIES}

It is very important that you keep the activities and information in this study confidential, since other will be participating in this research. Also, due to acquire many subjective responses, we require your honest information throughout the study.

\section{QUESTIONS}

Along the experimental process, if any questions or confusion come up at any time, we are encourage you to ask. Also, if you have any questions, or do not understand information on this form, please feel free to ask the experimenter. 


\section{PATICIPANT'S PERMISSION}

I have read the informed consent and fully understand the procedures and conditions of the project. I have had all my questions answered, and I hereby give my voluntary consent to be a participant in this research study. I agree to abide by the rules of the project. If I participate, I understand that I may withdraw from the study at any time.

If I have questions, I will contact: Principal Investigator: Thurmon E. Lockhart, Assistant Professor, Grado Department of Industrial and Systems Engineering, 231-9088. Chairman, Institutional Review Board for Research Involving Human Subjects: David Moore, 231-4991.

Signature of Participant

\section{Date}

\section{CONTACT}

If you feel you have not been treated according to the description of this form, or your rights as a participant have been violated during the course of this research, you may contact Dr. David M. Moore, Interim Chair of the Instructional Review Board Research Division at 231-4991 or moored@,vt.edu

May we contact you for future studies related to the vision/glare research? If yes, please provide your name, address, phone number, and/or email in the below space: 


\section{Vita: Haruetai Mekaroonreung hmekaroo@vt.edu}

\section{EDUCATION}

Bachelor of Engineering, Industrial Engineering, May 2000

Chulalongkorn University, Bangkok, Thailand

(First Class Honors with Gold Medal)

Diploma, June 1996

GFW High School, Winthrop, Mennesota, USA.

(Graduated Magna Cum Lauda)

\section{WORK EXPERIENCE AND SPECIAL TRAINING}

Graduate Research Assistance, "Assessment of Aging Visual Characteristics" Project, TOYOTA - Virginia Polytechnic Institute and State University, 2002

First Aid Training, National Safety Council, First Aid Institute, VA, 2002

Microsoft Visual Basic 6, Computer Center for Phamaceutical Sciences, Faculty of Pharmaceutical Sciences, Chulalongkorn University, July 2001, Bangkok

Analyst, Accenture (Former known as Andersen Consulting), Bangkok, October 2000 - June 2001

- Collected and created the BCP documents, Business Continuity Plan Project, Tesco Lotus, Thailand

- Conducted user training for Business Objects Program (Oracle Datawarehouse), Business Reporting System Project, Tesco Lotus, Thailand

- Collected data and analyzed annual performance, Key Performance Indicator Project, Electricity Generated Authority of Thailand (EGAT), Thailand

- Analyzed data as part of the financial model, Distribution Strategies Project, Bayer Company, Thailand

Associate Consultant for ISO9002 project, INFORCORT Company Limited, Bangkok, July - August 2000

Warrant of participating in the Student Internship Program (Thanakarn Kubarn Kumeung), March - April 2000, Bangkok Bank, Bangkok, Thailand

Internal Audit Training, System Audit Course, 1999 
Industrial Engineering Trainee, Department of Engineering, Boonrawd Brewery

Company Limited, Bangkok, March - May 1999

\section{HONORS}

- P.E.O. International Peace Scholarship, 2002

- Phi Beta Delta International Scholars, 2002

- Fulbright Scholarship Program for master's degree, 2001

- Outstanding Academic Honor Award, Chulalongkorn University Savings Cooperative Limited, 2000

- Gold Medal for the number one ranked student in the Industrial Engineering Department, Chulalongkorn University, Academic year 1999

- First Class Honor Graduate, Chulalongkorn University, Academic year 1999

- Academic Scholarship ( $1^{\text {st }}$ ranked student), The Siam Cement Group Public Company Limited, 1998

- Academic Honor Certificates, Faculty of Engineering, for academic years 1996, 97, 98

- Selected as an exchange student under AFS International and Intercultural Program, 1995-96

\section{PROFESSIONAL MEMBERSHIP}

- Human Factors and Ergonomics Society (HFES), 2001-02

- The American Society of Safety Engineers (ASSE), 2001-02 